\title{
On the Hermitian structures of the sequence of tangent bundles of an affine manifold endowed with a Riemannian metric
}

https://doi.org/10.1515/coma-2021-0128

Received August 29, 2021; accepted December 30, 2021

Abstract: Let $(M, \nabla,\langle\rangle$,$) be a manifold endowed with a flat torsionless connection \nabla$ and a Riemannian metric $\langle$,$\rangle and \left(T^{k} M\right)_{k \geq 1}$ the sequence of tangent bundles given by $T^{k} M=T\left(T^{k-1} M\right)$ and $T^{1} M=T M$. We show that, for any $k \geq 1, T^{k} M$ carries a Hermitian structure $\left(U_{k}, g_{k}\right)$ and a flat torsionless connection $\nabla^{k}$ and when $M$ is a Lie group and $(\nabla,\langle\rangle$,$) are left invariant there is a Lie group structure on each T^{k} M$ such that $\left(J_{k}, g_{k}, \nabla^{k}\right)$ are left invariant. It is well-known that $\left(T M, J_{1}, g_{1}\right)$ is Kähler if and only if $\langle$,$\rangle is Hessian, i.e, in$ each system of affine coordinates $\left(x_{1}, \ldots, x_{n}\right),\left\langle\partial_{x_{i}}, \partial_{x_{j}}\right\rangle=\frac{\partial^{2} \phi}{\partial_{x_{i}} \partial_{j}}$. Having in mind many generalizations of the Kähler condition introduced recently, we give the conditions on $(\nabla,\langle\rangle$,$) so that \left(T M, J_{1}, g_{1}\right)$ is balanced, locally conformally balanced, locally conformally Kähler, pluriclosed, Gauduchon, Vaisman or Calabi-Yau with torsion. Moreover, we can control at the level of $(\nabla,\langle\rangle$,$) the conditions insuring that some \left(T^{k} M, J_{k}, g_{k}\right)$ or all of them satisfy a generalized Kähler condition. For instance, we show that there are some classes of $(M, \nabla,\langle\rangle$,$) such that, for any k \geq 1,\left(T^{k} M, J_{k}, g_{k}\right)$ is balanced non-Kähler and Calabi-Yau with torsion. By carefully studying the geometry of $(M, \nabla,\langle\rangle$,$) , we develop a powerful machinery to build a large classes of$ generalized Kähler manifolds.

Keywords: Affine manifolds; Generalized Kähler Geometry; Hessian manifolds

MSC: 53C55; 53C05;53A15; 22E25

\section{Introduction}

Let $(N, J, g)$ be a complex manifold of real dimension $2 n, n \geq 2$, equipped with a Hermitian metric $g$. For any $\eta \in \Omega^{p}(N)$,

$$
J \eta\left(X_{1}, \ldots, X_{p}\right)=(-1)^{p} \eta\left(J X_{1}, \ldots, J X_{p}\right) \quad \text { and } \quad d^{c} \eta=-(-1)^{p} J^{-1} d J \eta, \quad X_{1}, \ldots, X_{p} \in \Gamma(T N) .
$$

The fundamental form is given by $\omega(.,)=.g\left(J\right.$., . ) and the Lee form is given by $\theta=J d^{\star} \omega=-d^{\star} \omega \circ J$, where for any $X_{1}, \ldots, X_{p-1} \in \Gamma(T N)$,

$$
d^{\star} \eta\left(X_{1}, \ldots, X_{p-1}\right)=-\sum_{i=1}^{2 n} \nabla_{E_{i}}^{L C} \eta\left(E_{i}, X_{1}, \ldots, X_{p-1}\right),
$$

$\nabla^{L C}$ is the Levi-Civita connection of $g$ and $\left(E_{1}, \ldots, E_{2 n}\right)$ is a local $g$-orthonormal frame. A fundamental class of Hermitian metrics is provided by Kähler metrics, satisfying $d \omega=0$. In literature, many generalizations of the Kähler condition have been introduced. Indeed, $(N, J, g)$ is called:

1. strongly Kähler with torsion or pluriclosed if $d d^{c} \omega=0$, i.e., $d J d J \omega=0$,

^Corresponding Author: Mohamed Boucetta: Université Cadi-Ayyad, Faculté des sciences et techniques, BP 549 Marrakech Maroc, E-mail: m.boucetta@uca.ac.ma 
2. balanced if $\theta=0$,

3. locally conformally balanced if $\theta$ is closed,

4. Gauduchon if $d^{\star} \theta=0$,

5. locally conformally Kähler if $d \omega=\frac{1}{n-1} \theta \wedge \omega$ and if, in addition, $\nabla^{L C} \theta=0$ then it is called Vaisman.

For general results about these generalized Kähler metrics, we refer the reader to $[1,3,4,6,10,11,22,25,27,28]$.

The Levi-Civita connection of $(N, g)$ is the only torsion free metric connection. In general, it does not preserve the complex structure $J$, this condition forcing the metric to be Kähler. Gauduchon proved in [12] that there exists and affine line of canonical Hermitian connections (they preserve both $J$ and $g$ ) passing through the Bismut connection and the Chern connection. The Bismut connection $\nabla^{B}$ (also known as Strominger connection) is the unique Hermitian connection with totally skew-symmetric torsion and the Chern connection $\nabla^{C}$ is the unique Hermitian connection whose torsion has trivial $(1,1)$-component. For any $X, Y, Z \in \Gamma(T N)$,

$$
\left\{\begin{array}{l}
g\left(\nabla_{X}^{B} Y, Z\right)=g\left(\nabla_{X}^{L C} Y, Z\right)+\frac{1}{2} d \omega(J X, J Y, J Z), \\
g\left(\nabla_{X}^{C} Y, Z\right)=g\left(\nabla_{X}^{L C} Y, Z\right)-\frac{1}{2} d \omega(J X, Y, Z) .
\end{array}\right.
$$

Let $R^{\tau}(X, Y)=\nabla_{[X, Y]}^{\tau}-\nabla_{X}^{\tau} \nabla_{Y}^{\tau}+\nabla_{Y}^{\tau} \nabla_{X}^{\tau}$ be the curvature tensor of $\nabla^{\tau}$. The Ricci form of $\nabla^{\tau}$ is given, for any $X, Y \in \Gamma(T N)$, by

$$
\rho^{\tau}(X, Y)=\frac{1}{2} \sum_{i=1}^{2 n} g\left(R^{\tau}(X, Y) E_{i}, J E_{i}\right),
$$

where $\left(E_{1}, \ldots, E_{2 n}\right)$ is a local $g$-orthonormal frame. It is known [1] that $\rho^{C}=\rho^{B}-d J \theta$. Hermitian structures satisfying $\operatorname{Hol}^{0}\left(\nabla^{B}\right) \subset \mathrm{SU}(n)$, or equivalently $\rho^{B}=0$, are known in literature as Calabi-Yau with torsion and appear in heterotic string theory, related to the Hull-Strominger system in six dimensions [17, 20, 24].

On the other hand, let $(M, \nabla,\langle\rangle$,$) be a manifold of dimension n$ endowed with a flat torsionless connection $\nabla$ and a Riemannian metric $\langle$,$\rangle . Actually, \nabla$ defines an affine structure on $M$, i.e., there exists on $M$ an atlas of charts such that all transition functions between charts are affine transformations of $\mathbb{R}^{n}$. Conversely, any affine atlas defines a flat torsionless connection. We refer to the charts of this atlas as affine coordinates.

Through-out this paper, we call such triple $(M, \nabla,\langle\rangle$,$) an affine-Riemann manifold, we denote by D$ the Levi-Civita connection of $\langle\rangle,, K(X, Y)=D_{[X, Y]}-D_{X} D_{Y}+D_{Y} D_{X}$ its curvature, $\gamma$ the difference tensor and $\gamma^{*}$ its adjoint given, for any $X, Y, Z \in \Gamma(T M)$, by

$$
\gamma_{X} Y=D_{X} Y-\nabla_{X} Y \quad \text { and } \quad\left\langle\gamma_{X}^{\star} Y, Z\right\rangle=\left\langle Y, \gamma_{X} Z\right\rangle .
$$

Their traces with respect to the metric are the vector fields given by

$$
\operatorname{tr}_{\langle,\rangle}(\gamma)=\sum_{i=1}^{n} \gamma_{E_{i}} E_{i} \text { and } \operatorname{tr}_{\langle,\rangle}\left(\gamma^{\star}\right)=\sum_{i=1}^{n} \gamma_{E_{i}}^{\star} E_{i},
$$

where $\left(E_{1}, \ldots, E_{n}\right)$ is a local $\langle$,$\rangle -orthonormal frame. The 1-form \alpha$ given, for any $X \in \Gamma(T M)$, by

$$
\alpha(X)=\left\langle\operatorname{tr}_{\langle,\rangle}\left(\gamma^{*}\right), X\right\rangle
$$

is closed (see Proposition 3.1) and it is known as the first Koszul form in the theory of Hessian manifolds. The vanishing of $\alpha$ is equivalent to the Riemannian volume being parallel with respect to $\nabla$. We introduce also the 1-form $\xi$ given, for any $X \in \Gamma(T M)$, by

$$
\xi(X)=\left\langle\operatorname{tr}_{\langle,\rangle}(\gamma), X\right\rangle .
$$

We call $\xi$ the adjoint Koszul form. These two 1-forms play an important role in this paper.

It is well-known (see [23]) that there is a Hermitian structure $\left(J_{1}, g_{1}\right)$ on $T M$ canonically associated to $(M, \nabla,\langle\rangle$,$) and \left(T M, J_{1}, g_{1}\right)$ is Kähler if and only if $\langle$,$\rangle is Hessian, i.e, in each system of affine coordinates$ $\left(x_{1}, \ldots, x_{n}\right)$ there exists a function $\phi$ such that $\left\langle\partial_{x_{i}}, \partial_{x_{j}}\right\rangle=\frac{\partial^{2} \phi}{\partial_{x_{i}} \partial_{x_{j}}}$. This is equivalent to $\langle$,$\rangle satisfying the$ Codazzi equation

$$
\nabla_{X}(\langle,\rangle)(Y, Z)=\nabla_{Y}(\langle,\rangle)(X, Z), \quad X, Y, Z \in \Gamma(T M) .
$$


We will see that (6) is equivalent to $\gamma=\gamma^{\star}$. Actually, there is also a flat torsionless connection $\nabla^{1}$ on $T M$ such that $\nabla^{1} J_{1}=0$. The affine-Riemann structure $\left(T M, \nabla^{1}, g_{1}\right)$ gives rise to a Hermitian structure $\left(T T M, J_{2}, g_{2}\right)$ and a flat torsionless connection $\nabla^{2}$ on TTM. By induction, we get a sequence of Hermitian structures $\left(T^{k} M, J_{k}, g_{k}\right)$ where $T^{k} M=T\left(T^{k-1} M\right)$ and $T^{1} M=T M$. Moreover, each $T^{k} M$ carries a flat torsionless connection $\nabla^{k}$ such that $\nabla^{k}\left(J_{k}\right)=0$.

The purpose of this paper is to explore the properties of this sequence of Hermitian structures and find the conditions on $(M, \nabla,\langle\rangle$,$) leading to some or all \left(T^{k} M, J_{k}, g_{k}\right)$ to satisfy one of the generalized Kähler conditions introduced above. This will lead to the construction of interesting classes of generalized Kähler manifolds. We find also a large class of Hermitian manifolds which are Calabi-Yau with torsion or with vanishing Chern Ricci form. We will show also that the study of the geometry of affine-Riemann manifolds is interesting in its own right and we will generalize some results obtained on Hessian manifolds.

Let us enumerate the main results of this paper and give its organization:

1. In Section 2, we define the sequence of Hermitian structures $\left(T^{k} M, J_{k}, g_{k}\right)$ and the sequence of flat torsionless connections $\nabla^{k}$ associated to an affine-Riemann manifold $(M, \nabla,\langle\rangle$,$) and we show that if M$ is a Lie group and $(\nabla,\langle\rangle$,$) are left invariant then there is on each T^{k} M$ a Lie group structure such that $\left(J_{k}, g_{k}, \nabla^{k}\right)$ are left invariant.

2. In Section 3, we give the useful tools for the study of the Hermitian manifolds $\left(T^{k} M, J_{k}, g_{k}\right)$. Namely, we compute the Levi-Civita connection $\nabla^{L C}$ of $\left(T M, g_{1}\right)$ and we show that the Lee form $\theta_{1}$ of $\left(T M, J_{1}, g_{1}\right)$ is given by means of the Koszul forms, namely, $\theta_{1}=\pi_{1}^{\star}(\alpha-\xi)$ where $\pi_{1}: T M \longrightarrow M$ is the canonical projection. We compute also the difference tensor for $\left(T M, \nabla^{1}, g_{1}\right)$ as well as its dual and we deduce by induction the Koszul forms $\alpha_{k}$ and $\xi_{k}$ and hence the Lee form of $\left(T^{k} M, \nabla^{k}, g_{k}\right)$. We give the conditions involving $\alpha, \xi, \operatorname{tr}_{\langle,\rangle}(\gamma), \operatorname{tr}_{\langle,\rangle}\left(\gamma^{*}\right)$ so that $\left(T M, J_{1}, g_{1}\right)$ is balanced, locally conformally balanced, Gauduchon, locally conformally Kähler or Vaisman.

3. In Section 4, we prove that $\left(T M, J_{1}, g_{1}\right)$ is pluriclosed if and only if the curvature $K$ of $\langle$,$\rangle satisfies, for$ any $X, Y \in \Gamma(T M)$,

$$
K(X, Y)=\gamma_{X}^{\star} \circ \gamma_{Y}-\gamma_{Y}^{\star} \circ \gamma_{X}
$$

It is known (see [23, Theorem $8.8 \mathrm{pp} .162]$ ) that if $M$ is compact, $\operatorname{tr}_{\langle,\rangle}\left(\gamma^{*}\right)=0$ and $\langle$,$\rangle is Hessian, i.e.,$ $\left(T M, J_{1}, g_{1}\right)$ is Kähler then $\nabla$ is the Levi-Civita of $\langle$,$\rangle . By using the splitting theorem of J. Cheeger and$ D. Gromoll (see for instance [5, Corollary $6.67 \mathrm{pp} .168]$ ), we prove that this result is still valid when we suppose that $M$ is compact, $\operatorname{tr}_{\langle,\rangle}\left(\gamma^{*}\right)=0, \nabla$ is complete and $\left(T M, J_{1}, g_{1}\right)$ is pluriclosed (see Theorem 4.1).

4. In Section 5, we compute the Bismut connection $\nabla^{B}$ and the Chern connection $\nabla^{C}$ of $\left(T M, J_{1}, g_{1}\right)$ and we give their the curvatures and their Ricci forms. We show that if $\operatorname{tr}_{\langle,\rangle}(\gamma)=0$ (resp. $\left.\operatorname{tr}_{\langle,\rangle}\left(\gamma^{\star}\right)=0\right)$ then the Ricci form $\rho^{B}$ (resp. $\rho^{C}$ ) of $\left(T M, J_{1}, g_{1}\right)$ vanish.

5. In Section 6, we remark first that if $\gamma=0$ then for any $k \geq 1$, the affine connection $\nabla^{k}$ is the Levi-Civita connection of $g_{k}$ and hence $\left(T^{k} M, J_{k}, g_{k}\right)$ is Kähler flat. Moreover, for $k_{0} \geq 1$ fixed, we show:

(a) If $k_{0} \geq 2$ then $\left(T^{k_{0}} M, J_{k_{0}}, g_{k_{0}}\right)$ is Kähler if and only if $\gamma=0$,

(b) $\left(T^{k_{0}} M, J_{k_{0}}, g_{k_{0}}\right)$ is locally conformally balanced if and only if $\left(T M, J_{1}, g_{1}\right)$ is locally conformally balanced and this is equivalent to $d \xi=0$,

(c) $\left(T^{k_{0}} M, J_{k_{0}}, g_{k_{0}}\right)$ is balanced if and only if $\operatorname{tr}_{\langle,\rangle}(\gamma)=\left(2^{k_{0}}-1\right) \operatorname{tr}_{\langle,\rangle}\left(\gamma^{*}\right)$ and in this case all the others $\left(T^{k} M, J_{k}, g_{k}\right)$ are locally conformally balanced and $\left(T^{k_{0}+1} M, J_{k_{0}+1}, g_{k_{0}+1}\right)$ is Calabi-Yau with torsion.

We express also in affine local coordinates the conditions on $\langle$,$\rangle so that \left(T M, J_{1}, g_{1}\right)$ is balanced or pluriclosed and we give many examples. We show that an affine-Riemann manifold $(M, \nabla,\langle\rangle$,$) so that$ $\left(T M, J_{1}, g_{1}\right)$ is Vaisman non-Kähler carries a codimension one totally geodesic foliation and $\langle$,$\rangle is flat$ when $\operatorname{dim} M=2$.

6. In Section 7, we study the class of affine-Riemann manifolds $(M, \nabla,\langle\rangle$,$) satisfying D(\gamma)=0$. We call the elements of this class rigid affine-Riemann manifolds. We show for this class that, for any $k \geq 1$, $\left(T^{k} M, J_{k}, g_{k}\right)$ is locally conformally balanced. By using a theorem by Kostant [19, Theorem 4], we show 
that when $M$ is simply-connected and $\langle$,$\rangle is complete then (M,\langle\rangle$,$) is a symmetric space and there is$ a connected Lie group $G$ which acts transitively and reducibly on $M$ by preserving both $\nabla$ and $\langle$, $\rangle$. We determine the elements of such class when $\operatorname{dim} M \leq 3$.

7. In Section 8, we study the class of affine-Riemann manifolds $(M, \nabla,\langle\rangle$,$) satisfying \operatorname{tr}_{\langle,\rangle}(\gamma)=\operatorname{tr}_{\langle,\rangle}\left(\gamma^{*}\right)=$ 0 . We call the elements of this class infinitely balanced affine-Riemann manifolds. Indeed, we prove that the condition $\operatorname{tr}_{\langle,\rangle}(\gamma)=\operatorname{tr}_{\langle,\rangle}\left(\gamma^{*}\right)=0$ and $\gamma \neq 0$ imply that, for any $k \geq 1,\left(T^{k} M, J_{k}, g_{k}\right)$ is balanced (non-Kähler when $k \geq 2$ ) and their Ricci forms $\rho_{k}^{C}$ and $\rho_{k}^{B}$ vanishes. Moreover, if $\gamma=\gamma^{\star}$, i.e, $\langle$,$\rangle is Hes-$ sian then the Ricci curvature of $\langle$,$\rangle is nonnegative and \left(T M, J_{1}, g_{1}\right)$ is Kähler Ricci-flat. In dimension 2, we show that $\operatorname{tr}_{\langle,\rangle}(\gamma)=\operatorname{tr}_{\langle,\rangle}\left(\gamma^{*}\right)=0$ implies that $\langle$,$\rangle is Hessian and the curvature is nonnegative.$ Moreover, if $\langle$,$\rangle is complete then \gamma=0$, i.e., $\nabla$ is the Levi-Civita of $\langle$,$\rangle . Non trivial examples of infinitely$ balanced affine-Riemann manifolds exist. We show that, for any $n \geq 2$, there is an affine-Riemann structure $(\nabla,\langle\rangle$,$) on S^{n} \times \mathbb{R}$ such that the corresponding $\gamma$ satisfies, $\gamma \neq 0, \gamma=\gamma^{*}$ and $\operatorname{tr}_{\langle,\rangle}(\gamma)=0$. Thus, $\left(T\left(S^{n} \times \mathbb{R}\right), J_{1}, g_{1}\right)$ is Kähler Ricci-flat and, for any $k \geq 2,\left(T^{k}\left(S^{n} \times \mathbb{R}\right), J_{k}, g_{k}\right)$ is balanced non-Kähler and their Ricci forms $\rho_{k}^{C}$ and $\rho_{k}^{B}$ vanish.

8. In Section 9, by using the classification of 3-dimensional real Novikov algebras, we give the infinitesimal part of a large class of generalized Kähler left invariant structures on some 6-dimensional Lie groups. We give also a large class of Calabi-Yau with torsion left invariant structures.

9. We think that one of the important contribution of this work is the development of a powerful machinery which permits the construction of large classes of examples of generalized Kähler manifolds (see Theorems 6.2, 6.3, 8.4, Corollaries 6.1, 7.1, Examples 1-5 and Tables 3-8).

\section{The canonical sequence of Hermitian structures associated to an affine-Riemann manifold}

In this section, we introduce the Hermitian structures and the affine connections on the sequence of tangent bundles associated to an affine-Riemann manifold and we show that these structures are left invariant when the affine-Riemann structure is left invariant.

\subsection{The Hermitian structures on the sequence of tangent bundles associated to an affine-Riemann manifold}

Let $(M, \nabla,\langle\rangle$,$) be an affine-Riemann manifold of dimension n$. Let $\pi_{1}: T M \longrightarrow M$ be the canonical projection and $Q: T T M \longrightarrow T M$ the connection map of $\nabla$ locally given by

$$
Q\left(\sum_{i=1}^{n} b_{i} \partial_{x_{i}}+\sum_{j=1}^{n} Z_{j} \partial_{\mu_{j}}\right)=\sum_{l=1}^{n}\left(Z_{l}+\sum_{i=1}^{n} \sum_{j=1}^{n} b_{i} \mu_{j} \Gamma_{i j}^{l}\right) \partial_{x_{l}},
$$

where $\left(x_{1}, \ldots, x_{n}\right)$ is a system of local coordinates, $\left(x_{1}, \ldots, x_{n}, \mu_{1}, \ldots, \mu_{n}\right)$ the associated system of coordinates on $T M$ and $\nabla_{\partial_{x_{i}}} \partial_{x_{j}}=\sum_{l=1}^{n} \Gamma_{i j}^{l} \partial_{x_{l}}$. Then

$$
T T M=\operatorname{ker} T \pi_{1} \oplus \operatorname{ker} Q .
$$

For $X \in \Gamma(T M)$, we denote by $X^{h}$ its horizontal lift and by $X^{v}$ its vertical lift. The flow of $X^{v}$ is given by $\Phi^{X}(t,(x, u))=(x, u+t X(x))$ and $X^{h}(x, u)=h^{(x, u)}(X(x))$, where $h^{(x, u)}: T_{X} M \longrightarrow \operatorname{ker} Q(x, u)$ is the inverse of the restriction of $d \pi_{1}$ to $\operatorname{ker} Q(x, u)$. Since the curvature of $\nabla$ vanishes, for any $X, Y \in \Gamma(T M)$,

$$
\left[X^{h}, Y^{h}\right]=[X, Y]^{h},\left[X^{h}, Y^{v}\right]=\left(\nabla_{X} Y\right)^{v} \text { and }\left[X^{v}, Y^{v}\right]=0 .
$$

The connection $\nabla^{1}$ on $T M$ given by

$$
\nabla^{1}{ }_{X^{h}} Y^{h}=\left(\nabla_{X} Y\right)^{h}, \nabla_{X^{h}}^{1} Y^{v}=\left(\nabla_{X} Y\right)^{v} \text { and } \quad \nabla^{1}{ }_{X^{v}} Y^{h}=\nabla_{X^{v}}^{1} Y^{v}=0,
$$


for any $X, Y \in \Gamma(T M)$, is flat torsionless and defines an affine structure on $T M$. The tensor field $J_{1}: T T M \longrightarrow$ TTM given by $J_{1} X^{h}=X^{v}$ and $J_{1} X^{v}=-X^{h}$ satisfies $J_{1}^{2}=-\operatorname{Id}_{T T M}, \nabla^{1}\left(J_{1}\right)=0$ and hence defines a complex structure on $T M$.

On the other hand, we define on $T M$ a Riemannian metric $g_{1}$ by

$$
g_{1}\left(X^{h}, Y^{h}\right)=\langle X, Y\rangle \circ \pi_{1}, g_{1}\left(X^{v}, Y^{v}\right)=\langle X, Y\rangle \circ \pi_{1} \quad \text { and } \quad g_{1}\left(X^{h}, Y^{v}\right)=0, \quad X, Y \in \Gamma(T M) .
$$

This metric is Hermitian with respect to $J_{1}$ and its fundamental form $\omega=g_{1}\left(J_{1} .\right.$, .) satisfies

$$
\omega\left(X^{h}, Y^{h}\right)=\omega\left(X^{v}, Y^{v}\right)=0 \quad \text { and } \quad \omega\left(X^{h}, Y^{v}\right)=-\omega\left(Y^{v}, X^{h}\right)=\langle X, Y\rangle \circ \pi_{1}, \quad X, Y \in \Gamma(T M) .
$$

Actually, we have a sequence of Hermitian structures. The affine-Riemann manifold $\left(T M, \nabla^{1}, g_{1}\right)$ gives rise to a Hermitian structure $\left(T T M, J_{2}, g_{2}\right)$ and a flat torsionless connection $\nabla^{2}$ on TTM. By induction, we get a sequence of Hermitian structures $\left(T^{k} M, J_{k}, g_{k}\right)$ where $T^{k} M=T\left(T^{k-1} M\right)$ and $T^{1} M=T M$. Moreover, each $T^{k} M$ carries a flat torsionless connection $\nabla^{k}$ such that $\nabla^{k}\left(J_{k}\right)=0$.

\subsection{The canonical sequence of Hermitian structures associated to a left invariant affine-Riemann structure}

Let $(G, \nabla,\langle\rangle$,$) be an affine-Riemann manifold such that G$ is a connected Lie group and $(\nabla,\langle\rangle$,$) are left$ invariant. Let $\left(\mathfrak{g}=T_{e} G,[],\right)$ be the Lie algebra of $G$. For any $a \in \mathfrak{g}$, we denote by $a^{-}$the left invariant vector field on $G$ associated to $a$. The affine connection $\nabla$ defines a product $\bullet$ on $\mathfrak{g}$ by

$$
(a \bullet b)^{-}=\left(\nabla a^{-} b^{-}\right)(e), \quad a, b \in \mathfrak{g} .
$$

This product is Lie-admissible, i.e., $a \bullet b-b \bullet a=[a, b]$ and left symmetric, i.e., for any $a, b, c \in \mathfrak{g}$,

$$
\operatorname{ass}(a, b, c)=\operatorname{ass}(b, a, c),
$$

where ass $(a, b, c)=(a \bullet b) \bullet c-a \bullet(b \bullet c)$. This is equivalent to $\mathrm{L}: \mathfrak{g} \longrightarrow \operatorname{End}(\mathfrak{g}), a \mapsto \mathrm{L}_{a}$ is a representation of Lie algebras, where $\mathrm{L}_{a} b=a \bullet b$,

Put $\Phi(\mathfrak{g})=\mathfrak{g} \times \mathfrak{g}$ and define on $\Phi(\mathfrak{g})$ a product *, a bracket [ , $]_{\Phi}$, an isomorphism $J: \Phi(\mathfrak{g}) \longrightarrow \Phi(\mathfrak{g})$ and a scalar product $\langle,\rangle_{\phi}$ by

$$
\begin{aligned}
& (a, b) \star(c, d)=(a \bullet c, a \bullet d), \quad[(a, b),(c, d)]_{\Phi}=([a, c], a \bullet d-c \bullet b), \\
& J(a, b)=(-b, a) \quad \text { and } \quad\langle(a, b),(c, d)\rangle_{\Phi}=\langle a, c\rangle+\langle b, d\rangle,
\end{aligned}
$$

for any $(a, b),(c, d) \in \Phi(\mathfrak{g})$. It is easy to check that * is left symmetric and hence its commutator which is [ , ] $]_{\Phi}$ is a Lie bracket. Moreover, for any $(a, b),(c, d) \in \Phi(\mathfrak{g})$,

$$
\left.N_{J}((a, b),(c, d))=[J(a, b), J(c, d)]_{\Phi}-J[(a, b), J(c, d)]_{\Phi}-J U(a, b),(c, d)\right]_{\Phi}-[(a, b),(c, d)]_{\Phi}=0 .
$$

On the other hand, let $\rho: G \longrightarrow \mathrm{GL}(\mathfrak{g})$ be the homomorphism of groups such that $d_{e} \rho=\mathrm{L}$ and consider the product on $G \times \mathfrak{g}$ given by

$$
(p, a) .(q, b)=\left(p q, b+\rho\left(q^{-1}\right)(a)\right), \quad p, q \in G, a, b \in \mathfrak{g} .
$$

Proposition 2.1. $(G \times \mathfrak{g},$.$) is a Lie group whose Lie algebra is \left(\Phi(\mathfrak{g}),[,]_{\Phi}\right)$.

Proof. For any $(p, a),(q, b) \in G \times \mathfrak{g},(p, a)^{-1}=\left(p^{-1},-\rho(p)(a)\right)$ and

$$
\begin{aligned}
L_{(p, a)} \circ R_{\left(p^{-1},-\rho(p)(a)\right)}(q, b) & =(p, a)(q, b)\left(p^{-1},-\rho(p)(a)\right) \\
& =\left(p q, b+\rho\left(q^{-1}\right)(a)\right)\left(p^{-1},-\rho(p)(a)\right) \\
& =\left(p q p^{-1},-\rho(p)(a)+\rho(p)(b)+\rho\left(p q^{-1}\right)(a)\right) .
\end{aligned}
$$


So, for any $(X, b),(Y, c) \in T_{(e, 0)}(G \times \mathfrak{g})$,

$$
\operatorname{Ad}_{(p, a)}(X, b)=\left(\operatorname{Ad}_{p} X, \rho(p)(b)-\rho(p)(X \bullet a)\right)
$$

and hence

$$
[(X, b),(Y, c)]=([X, Y], X \bullet c-Y \bullet b) .
$$

The triple $\left(J,\langle,\rangle_{\Phi},{ }^{\star}\right)$ induces a left invariant triple $\left(J_{0}, g_{0}, \nabla^{0}\right)$ on $G \times \mathfrak{g}$ satisfying $J_{0}(a, b)^{-}=(-b, a)^{-}, g_{0}\left((a, b)^{-},(c, d)^{-}\right)=\langle(a, b),(c, d)\rangle_{\Phi} \quad$ and $\quad \nabla_{(a, b)^{-}}^{0}(c, d)^{-}=\left((a, b)^{\star}(c, d)\right)^{-}, \quad a, b, c, d \in \mathfrak{g}$. Thus $\left(G \times \mathfrak{g}, J_{0}, g_{0}\right)$ is a left invariant Hermitian structure and $\nabla^{0}$ is a left invariant flat torsionless connection on $G \times \mathfrak{g}$.

Denote by $\Theta: T G \longrightarrow G \times \mathfrak{g}$ the identification $X_{p} \longrightarrow\left(p, T_{p} L_{p^{-1}} X_{p}\right)$.

Theorem 2.1. Let $\left(T G, J_{1}, g_{1}\right)$ be the canonical Hermitian structure associated to $(G, \nabla,\langle\rangle$,$) and \nabla^{1}$ the associated canonical affine connection. Then $\Theta$ sends $\left(J_{1}, g_{1}, \nabla^{1}\right)$ to $\left(J_{0}, g_{0}, \nabla^{0}\right)$, i.e., for any $X, Y \in \Gamma(T G)$,

$$
g(X, Y)=g_{0}\left(\Theta_{\star} X, \Theta_{\star} Y\right), \Theta_{\star}\left(J_{1} X\right)=J_{0} \Theta_{\star} X \text { and } \Theta_{*}\left(\nabla_{X}^{1} Y\right)=\nabla_{\Theta * X}^{0} \Theta_{\star} Y .
$$

To prove this theorem, we need some preparation.

Proposition 2.2. Let $(G, \mathcal{D})$ be a Lie group endowed with a left invariant connection, $\tau:[0,1] \longrightarrow G$ a curve and $V:[0,1] \longrightarrow T G$ a vector field along $\tau$. We define $\tau^{\ell}:[0,1] \longrightarrow \mathfrak{g}$ and $W:[0,1] \longrightarrow \mathfrak{g}$ by

$$
\tau^{\ell}(t)=T_{\tau(t)} L_{\tau(t)^{-1}}\left(\tau^{\prime}(t)\right) \quad \text { and } \quad W(t)=T_{\tau(t)} L_{\tau(t)^{-1}}(V(t)) .
$$

Then $V$ is parallel along $\tau$ with respect $\mathcal{D}$ if and only if, for any $t \in[0,1]$,

$$
W^{\prime}(t)+\tau^{\ell}(t) \bullet W(t)=0,
$$

where $u \bullet v=\left(\mathcal{D}_{u^{-}} v^{-}\right)(e)$.

Proof. We consider $\left(u_{1}, \ldots, u_{n}\right)$ a basis of $\mathfrak{g}$ and $\left(X_{1}, \ldots, X_{n}\right)$ the corresponding left invariant vector fields. Then

$$
\tau^{\ell}(t)=\sum_{i=1}^{n} \tau_{i}^{\ell}(t) u_{i}, W(t)=\sum_{i=1}^{n} W_{i}(t) u_{i}, \tau^{\prime}(t)=\sum_{i=1}^{n} \tau_{i}^{\ell}(t) X_{i}, \quad V(t)=\sum_{i=1}^{n} W_{i}(t) X_{i} .
$$

Then

$$
\begin{aligned}
\mathcal{D}_{t} V(t) & =\sum_{i=1}^{n} W_{i}^{\prime}(t) X_{i}+\sum_{i=1}^{n} W_{i}(t) \mathcal{D}_{\tau^{\prime}(t)} X_{i} \\
& =\sum_{i=1}^{n} W_{i}^{\prime}(t) X_{i}+\sum_{i, j=1}^{n} W_{i}(t) \tau_{j}^{\ell}(t) \mathcal{D}_{X_{j}} X_{i} \\
& =\sum_{i=1}^{n} W_{i}^{\prime}(t) X_{i}+\sum_{i, j=1}^{n} W_{i}(t) \tau_{j}^{\ell}(t)\left(u_{j} \bullet u_{i}\right)^{-} \\
& =\left(W^{\prime}(t)+\tau^{\ell}(t) \bullet W(t)\right)^{-}
\end{aligned}
$$

and the result follows.

Proposition 2.3. Let $(G, \nabla,\langle\rangle$,$) be a left invariant affine-Riemann structure on a connected Lie group. Then:$

1. For any $X \in T_{p} G$ and any $a \in \mathfrak{g}$,

$$
T \Theta\left(X^{v}\right)(p, a)=\left(0, T_{p} L_{p^{-1}}(X)\right) \quad \text { and } \quad T \Theta\left(X^{h}\right)(p, a)=\left(X,-T_{p} L_{p^{-1}}(X) \bullet a\right) .
$$


2. For any $(a, b) \in \mathfrak{g} \times \mathfrak{g},(a, b)^{-}=T \Theta\left(\left(a^{-}\right)^{h}+\left(b^{-}\right)^{v}\right)$.

Proof. The first relation is obvious. Recall that the horizontal lift of $X$ at $u_{p} \in T G$ is given by

$$
X^{h}\left(u_{p}\right)=\frac{d}{d t} \mid t=0
$$

where $V:[0,1] \longrightarrow T G$ is the $\nabla$-parallel vector field along a curve $\tau:[0,1] \longrightarrow G$ such that $\tau(0)=p$, $\tau^{\prime}(0)=X$ and $V(0)=u_{p}$. Put $a=T_{p} L_{p^{-1}}\left(u_{p}\right)$. By virtue of Proposition 2.2,

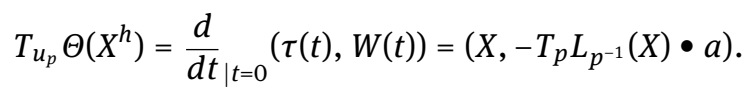

2. For any $p \in G$ and $u \in \mathfrak{g}$, we have

$$
\begin{aligned}
(a, b)^{-}(p, u) & =T_{(e, 0)} L_{(p, u)}(a, b) \\
& =\left.\frac{d}{d t}\right|_{t=0}(p, u)(\exp (t a), t b) \\
& =\left.\frac{d}{d t}\right|_{t=0}(p \exp (t a), t b+\rho(\exp (-t a))(u)) \\
& =\left(a^{-}(p), b-a \bullet u\right) \\
& =\left(a^{-}(p),-T_{p} L_{p^{-1}}\left(a^{-}(p)\right) \bullet u\right)+\left(0, T_{p} L_{p^{-1}}\left(b^{-}(p)\right)\right. \\
& =T \Theta\left(a^{-}\right)^{h}(p, u)+T \Theta\left(b^{-}\right)^{v}(p, u) .
\end{aligned}
$$

\subsubsection{Proof of Theorem 2.1}

Proof. By virtue of Proposition 2.3,

$$
\begin{aligned}
J_{0}(a, b)^{-} & =(-b, a)^{-}=-T \Theta\left(b^{-}\right)^{h}+T \Theta\left(a^{-}\right)^{v}, \\
& =T \Theta J_{1}\left(\left(b^{-}\right)^{v}\right)+T \Theta J_{1}\left(\left(a^{-}\right)^{h}\right) \\
& =T \Theta J_{1}(T \Theta)^{-1}(a, b)^{-} .
\end{aligned}
$$

The other relations can be deduced similarly.

\section{Basic tools for the study of the canonical sequence of Hermitian structures associated to an affine-Riemann manifold}

Trough-out this section and the next one, $(M, \nabla,\langle\rangle$,$) is an affine-Riemann manifold of dimension n, D$ the Levi-Civita connection of $\langle$,$\rangle and \mu$ the Riemannian volume. Let $\left(T^{k} M, J_{k}, g_{k}, \nabla^{k}\right), k \geq 1$, be the canonical sequence of Hermitian structures associated to $(M, \nabla,\langle\rangle$,$) endowed with the sequence of flat torsionless$ connections. For any $k \geq 1$, we denote by $\pi_{k}: T^{k} M \longrightarrow T^{k-1} M$ the canonical projection. We consider the difference tensor $\gamma$ and its dual $\gamma^{\star}$ given by (2), their traces given by (3) and the Koszul forms $\alpha$ and $\xi$ given by (4) and (5).

Since both $\nabla$ and $D$ are torsionless, $\gamma$ is symmetric and it is easy to check that, for any $X, Y, Z, U \in \Gamma(T M)$,

$$
\nabla_{X}(\langle,\rangle)(Y, Z)=\left\langle\gamma_{X} Y+\gamma_{X}^{\star} Y, Z\right\rangle,\left\langle D_{X}(\gamma)(Y, Z), U\right\rangle=\left\langle D_{X}\left(\gamma^{\star}\right)(Y, U), Z\right\rangle .
$$

Since $\nabla$ is flat, the curvature $K(X, Y)=D_{[X, Y]}-\left[D_{X}, D_{Y}\right]$ of $\langle$, $\rangle$ satisfies

$$
K(X, Y) Z=D_{Y}(\gamma)(X, Z)-D_{X}(\gamma)(Y, Z)+\left[\gamma_{X}, \gamma_{Y}\right] Z
$$


From the relation $K(X, Y)^{\star}=-K(X, Y)$, we deduce that

$$
K(X, Y) Z=D_{X}\left(\gamma^{\star}\right)(Y, Z)-D_{Y}\left(\gamma^{\star}\right)(X, Z)+\left[\gamma_{X}^{\star}, \gamma_{Y}^{\star}\right] Z
$$

and hence

$$
D_{Y}\left(\gamma+\gamma^{\star}\right)(X, Z)-D_{X}\left(\gamma+\gamma^{\star}\right)(Y, Z)=\left[\gamma_{X}^{\star}, \gamma_{Y}^{*}\right] Z-\left[\gamma_{X}, \gamma_{Y}\right] Z .
$$

The first Koszul form $\alpha$ satisfies the following properties.

Proposition 3.1. For any $X \in \Gamma(T M)$,

$$
\nabla_{X} \mu=\left\langle X, \operatorname{tr}_{\langle,\rangle}\left(\gamma^{\star}\right)\right\rangle \mu=\operatorname{tr}\left(\gamma_{X}\right) \mu=\alpha(X) \mu .
$$

In particular, the first Koszul 1-form $\alpha$ is closed.

Proof. Let $\left(E_{1}, \ldots, E_{n}\right)$ be a local $\langle$,$\rangle -orthonormal frame.$

$$
\begin{aligned}
\nabla_{X} \mu\left(E_{1}, \ldots, E_{n}\right) & =-\sum_{i=1}^{n} \mu\left(E_{1}, \ldots, \nabla_{X} E_{i}, \ldots, E_{n}\right)=-\sum_{i=1}^{n}\left\langle\nabla_{X} E_{i}, E_{i}\right\rangle=\frac{1}{2} \sum_{i=1}^{n} \nabla_{X}(\langle,\rangle)\left(E_{i}, E_{i}\right) \\
& \stackrel{(12)}{=} \frac{1}{2}\left\langle\gamma_{X} E_{i}+\gamma_{X}^{\star} E_{i}, E_{i}\right\rangle=\operatorname{tr}\left(\gamma_{X}\right)=\left\langle\operatorname{tr}_{\langle,\rangle}\left(\gamma^{\star}\right), X\right\rangle .
\end{aligned}
$$

The fact that $\alpha$ is closed is a consequence of the fact that, for any $X, Y \in \Gamma(T M)$,

$$
\left(\nabla_{[X, Y]}-\nabla_{X} \nabla_{Y}+\nabla_{Y} \nabla_{X}\right) \mu=0 .
$$

Proposition 3.2. The differential of the fundamental form $\omega$ associated to $\left(T M, J_{1}, g_{1}\right)$ is given by

$$
d \omega\left(X^{h}, Y^{h}, Z^{h}\right)=d \omega\left(X^{v}, Y^{v}, Z^{v}\right)=d \omega\left(X^{h}, Y^{v}, Z^{v}\right)=0 \quad \text { and } \quad d \omega\left(X^{h}, Y^{h}, Z^{v}\right)=\left\langle\gamma_{X}^{\star} Y-\gamma_{Y}^{\star} X, Z\right\rangle \circ \pi_{1},
$$

for any $X, Y, Z \in \Gamma(T M)$. Hence

$\left(J_{1} d \omega\right)\left(X^{h}, Y^{h}, Z^{h}\right)=\left(J_{1} d \omega\right)\left(X^{v}, Y^{v}, Z^{\nu}\right)=\left(J_{1} d \omega\right)\left(X^{h}, Y^{h}, Z^{\nu}\right)=0 \quad$ and $\quad\left(J_{1} d \omega\right)\left(X^{v}, Y^{v}, Z^{h}\right)=-\left\langle\gamma_{X}^{\star} Y-\gamma_{Y}^{\star} X, Z\right\rangle \circ \pi_{1}$.

Proof. From (7) and (9), we have obviously $d \omega\left(X^{h}, Y^{h}, Z^{h}\right)=d \omega\left(X^{v}, Y^{v}, Z^{v}\right)=d \omega\left(X^{h}, Y^{v}, Z^{v}\right)=0$. On the other hand,

$$
\begin{aligned}
d \omega\left(X^{h}, Y^{h}, Z^{\nu}\right) & =X .\langle Y, Z\rangle \circ \pi_{1}-Y .\langle X, Z\rangle \circ \pi_{1}-\langle[X, Y], Z\rangle \circ \pi_{1}-\left\langle\nabla_{X} Z, Y\right\rangle \circ \pi_{1}+\left\langle\nabla_{Y} Z, X\right\rangle \circ \pi_{1} \\
& =\nabla_{X}(\langle,\rangle)(Y, Z) \circ \pi_{1}-\nabla_{Y}(\langle,\rangle)(X, Z) \circ \pi_{1}, \\
& \stackrel{(12)}{=}\left\langle\gamma_{X}^{\star} Y-\gamma_{Y}^{\star} X, Z\right\rangle \circ \pi_{1} .
\end{aligned}
$$

As an immediate consequence of the expression of $d \omega$, the proof above, (14) and (15), we get the following result which sums up some of the important properties of Hessian manifolds (see [23]). Recall that a Hessian manifold is an affine-Riemann manifold $(M, \nabla,\langle\rangle$,$) such that in any affine coordinates \left(x_{1}, \ldots, x_{n}\right)$ there exists a function $\phi$ such that $\left\langle\partial_{x_{i}}, \partial_{x_{j}}\right\rangle=\frac{\partial^{2} \phi}{\partial x_{i} \partial x_{j}}$ for any $i, j \in\{1, \ldots, n\}$. This is equivalent to $\langle$,$\rangle satisfying$ the Codazzi equation (6).

Corollary 3.1. $\left(T M, J_{1}, g_{1}\right)$ is Kähler if and only if $(M, \nabla,\langle\rangle$,$) is Hessian manifold which is also equivalent to$ $\gamma=\gamma^{\star}$. In this case,

$$
D_{Y}(\gamma)(X, Z)=D_{X}(\gamma)(Y, Z) \quad \text { and } \quad K(X, Y)=\left[\gamma_{X}, \gamma_{Y}\right], \quad X, Y, Z \in \Gamma(T M)
$$

Let us compute now the Levi-Civita connection $\nabla^{L C}$ of $\left(T M, J_{1}, g_{1}\right)$ and its Lee form. 
Proposition 3.3. For any $X, Y \in \Gamma(T M)$,

$$
\nabla_{X^{h}}^{L C} Y^{h}=\left(D_{X} Y\right)^{h}, \nabla_{X^{v}}^{L C} Y^{v}=-\frac{1}{2}\left(\gamma_{X}^{\star} Y+\gamma_{Y}^{\star} X\right)^{h}, \nabla_{X^{v}}^{L C} Y^{h}=\left(\gamma_{Y}^{S} X\right)^{v} \quad \text { and } \quad \nabla_{X^{h}}^{L C} Y^{v}=\left(D_{X} Y\right)^{v}-\left(\gamma_{X}^{a} Y\right)^{v},
$$

where

$$
\gamma^{a}=\frac{1}{2}\left(\gamma-\gamma^{\star}\right) \quad \text { and } \quad \gamma^{s}=\frac{1}{2}\left(\gamma+\gamma^{\star}\right) .
$$

Proof. Let $X, Y, Z \in \Gamma(T M)$. From the Koszul formula and (7), we have obviously

$$
2 g\left(\nabla_{X^{h}}^{L C} Y^{h}, Z^{v}\right)=2 g\left(\nabla_{X^{v}}^{L C} Y^{v}, Z^{v}\right)=2 g\left(\nabla_{X^{v}}^{L C} Y^{h}, Z^{h}\right)=0 .
$$

On the other hand,

$$
\begin{aligned}
2 g\left(\nabla_{X^{h}}^{L C} Y^{h}, Z^{h}\right) & =2\left\langle D_{X} Y, Z\right\rangle \circ \pi_{1}, \\
2 g\left(\nabla_{X^{v}}^{L C} Y^{v}, Z^{h}\right) & =-Z .\langle X, Y\rangle \circ \pi_{1}+\left\langle\nabla_{Z} X, Y\right\rangle \circ \pi_{1}+\left\langle\nabla_{Z} Y, X\right\rangle \circ \pi_{1} \\
& =-\nabla_{Z}(\langle,\rangle)(X, Y) \circ \pi_{1} \\
& \stackrel{(12)}{=}-\left\langle\gamma_{Z} X+\gamma_{Z}^{\star} X, Y\right\rangle \circ \pi_{1}, \\
& =-\left\langle\gamma_{X}^{\star} Y+\gamma_{Y}^{\star} X, Z\right\rangle \circ \pi_{1}, \\
2 g\left(\nabla_{X^{v}}^{L C} Y^{h}, Z^{v}\right) & =Y .\langle X, Z\rangle \circ \pi_{1}-\left\langle\nabla_{Y} X, Z\right\rangle \circ \pi_{1}-\left\langle\nabla_{Y} Z, X\right\rangle \circ \pi_{1}, \\
& =\nabla_{Y}(\langle,\rangle)(X, Z) \circ \pi_{1} \\
& \stackrel{(12)}{=}\left\langle\gamma_{Y} X+\gamma_{Y}^{\star} X, Z\right\rangle \circ \pi_{1} .
\end{aligned}
$$

Proposition 3.4. The Lee form $\theta_{1}$ of $\left(T M, J_{1}, g_{1}\right)$ is given by

$$
\theta_{1}=\pi_{1}^{\star}(\alpha-\xi),
$$

where $\alpha$ and $\xi$ are the Koszul forms of $(M, \nabla,\langle\rangle$,$) . In particular, \left(T M, J_{1}, g_{1}\right)$ is balanced if and only if $\alpha=\xi$ which is also equivalent to

$$
\operatorname{tr}_{\langle,\rangle}\left(\gamma^{\star}-\gamma\right)=0
$$

Moreover, $\left(T M, J_{1}, g_{1}\right)$ is locally conformally balanced if and only if $d \xi=0$.

Proof. Let $\left(E_{1}, \ldots, E_{n}\right)$ be a local $\langle$,$\rangle -orthonormal frame. Having in mind the expressions of \nabla^{L C}$ given in the last proposition, for any $X \in \Gamma(T M)$,

$$
\begin{aligned}
-d^{\star} \omega\left(X^{h}\right) & =\sum_{i=1}^{n}\left(\nabla_{E_{i}^{h}}^{L C} \omega\left(E_{i}^{h}, X^{h}\right)+\nabla_{E_{i}^{v}}^{L C} \omega\left(E_{i}^{v}, X^{h}\right)\right) \\
& =\sum_{i=1}^{n}\left(E_{i}^{h} \cdot \omega\left(E_{i}^{h}, X^{h}\right)-\omega\left(\nabla_{E_{i}^{h}}^{L C} E_{i}^{h}, X^{h}\right)-\omega\left(E_{i}^{h}, \nabla_{E_{i}^{h}}^{L C} X^{h}\right)+E_{i}^{v} \cdot \omega\left(E_{i}^{v}, X^{h}\right)-\omega\left(\nabla_{E_{i}^{v}}^{L C} E_{i}^{v}, X^{h}\right)-\omega\left(E_{i}^{v}, \nabla_{E_{i}^{v}}^{L C} X^{h}\right)\right) \\
& =0 . \\
-d^{\star} \omega\left(X^{v}\right)= & \sum_{i=1}^{n}\left(\nabla_{E_{i}^{h}}^{L C} \omega\left(E_{i}^{h}, X^{v}\right)+\nabla_{E_{i}^{v}}^{L C} \omega\left(E_{i}^{v}, X^{v}\right)\right) \\
& =\sum_{i=1}^{n}\left(E_{i}^{h} \cdot \omega\left(E_{i}^{h}, X^{v}\right)-\omega\left(\nabla_{E_{i}^{h}}^{L C} E_{i}^{h}, X^{v}\right)-\omega\left(E_{i}^{h}, \nabla_{E_{i}^{h}}^{L C} X^{v}\right)+E_{i}^{v} \cdot \omega\left(E_{i}^{v}, X^{v}\right)-\omega\left(\nabla_{E_{i}^{v}}^{L C} E_{i}^{v}, X^{v}\right)-\omega\left(E_{i}^{v}, \nabla_{E_{i}^{v}}^{L C} X^{v}\right)\right) \\
& =\sum_{i=1}^{n}\left(E_{i} \cdot\left\langle E_{i}, X\right\rangle \circ \pi_{1}-\left\langle D_{E_{i}} E_{i}, X\right\rangle \circ \pi_{1}-\left\langle E_{i}, D_{E_{i}} X\right\rangle \circ \pi_{1}+\frac{1}{2}\left\langle E_{i}, \gamma_{E_{i}} X-\gamma_{E_{i}}^{\star} X\right\rangle \circ \pi_{1}\right.
\end{aligned}
$$




$$
\begin{aligned}
& \left.+\left\langle\gamma_{E_{i}}^{\star} E_{i}, X\right\rangle \circ \pi_{1}-\frac{1}{2}\left\langle\gamma_{E_{i}}^{\star} X+\gamma_{X}^{\star} E_{i}, E_{i}\right\rangle \circ \pi_{1}\right) \\
& =\left\langle\operatorname{tr}_{\langle,\rangle}\left(\gamma^{\star}\right)-\operatorname{tr}_{\langle,\rangle}(\gamma), X\right\rangle \circ \pi_{1} .
\end{aligned}
$$

Finally,

$$
\theta_{1}\left(X^{h}\right)=-d^{\star} \omega\left(J_{1} X^{h}\right)=\left\langle\operatorname{tr}_{\langle,\rangle}\left(\gamma^{\star}\right)-\operatorname{tr}_{\langle,\rangle}(\gamma), X\right\rangle \circ \pi_{1}, \theta_{1}\left(X^{v}\right)=0
$$

and we get the desired formula. Moreover, since $\alpha$ is closed then $d \theta_{1}=0$ if and only if $d \xi=0$.

Proposition 3.5. We have

$$
d^{\star} \theta_{1}=d^{\star}(\alpha-\xi) \circ \pi_{1}-\left\langle\operatorname{tr}_{\langle,\rangle}\left(\gamma^{\star}\right)-\operatorname{tr}_{\langle,\rangle}(\gamma), \operatorname{tr}_{\langle,\rangle}\left(\gamma^{\star}\right)\right\rangle \circ \pi_{1}
$$

and hence $\left(T M, J_{1}, g_{1}\right)$ is Gauduchon if and only if

$$
d^{\star}(\alpha-\xi)=\left|\operatorname{tr}_{\langle,\rangle}\left(\gamma^{\star}\right)\right|^{2}-\left\langle\operatorname{tr}_{\langle,\rangle}\left(\gamma^{\star}\right), \operatorname{tr}_{\langle,\rangle}(\gamma)\right\rangle .
$$

Proof. A straightforward computation using the definition of the divergence and the expressions of $\nabla^{L C}$.

Proposition 3.6. 1. (TM, $\left.J_{1}, g_{1}\right)$ is locally conformally Kähler if and only if, for any $X, Y \in \Gamma(T M)$,

$$
(n-1)\left(\gamma_{X}^{\star} Y-\gamma_{Y}^{\star} X\right)=\theta_{0}(X) Y-\theta_{0}(Y) X,
$$

where $\theta_{0}=\alpha-\xi$.

2. (TM, $\left.J_{1}, g_{1}\right)$ is Vaisman if and only if (17) holds and the vector field $\Pi:=\operatorname{tr}_{\langle,\rangle}\left(\gamma^{*}-\gamma\right)$ is parallel with respect to both $D$ and $\nabla$.

Proof. (TM, $\left.J_{1}, g_{1}\right)$ is locally conformally Kähler if and only if $(n-1) d \omega=\theta_{1} \wedge \omega$. For any $X, Y, Z \in \Gamma(T M)$,

$$
\begin{aligned}
\theta_{1} \wedge \omega\left(X^{h}, Y^{h}, Z^{h}\right) & =\theta_{1} \wedge \omega\left(X^{v}, Y^{v}, Z^{v}\right)=0, \\
\theta_{1} \wedge \omega\left(X^{h}, Y^{h}, Z^{v}\right) & =\theta_{1}\left(X^{h}\right)\langle Y, Z\rangle \circ \pi_{1}-\theta_{1}\left(Y^{h}\right)\langle X, Z\rangle \circ \pi_{1} \\
& =\theta_{0}(X) \circ \pi_{1}\langle Y, Z\rangle \circ \pi_{1}-\theta_{0}(Y) \circ \pi_{1}\langle X, Z\rangle \circ \pi_{1}, \\
\theta_{1} \wedge \omega\left(X^{h}, Y^{v}, Z^{v}\right) & =0
\end{aligned}
$$

and the first assertion follows by virtue of Proposition 3.2.

2. ( $T M, J_{1}, g_{1}$ ) is Vaisman if and only if it is locally conformally Kähler and $\nabla^{L C} \theta_{1}=0$. Now, by using Proposition 3.3, for any $X, Y \in \Gamma(T M)$,

$$
\nabla_{X^{h}}^{L C}\left(\theta_{1}\right)\left(Y^{h}\right)=\left(D_{X}\left(\theta_{0}\right)(Y)\right)^{h}, \nabla_{X^{h}}^{L C}\left(\theta_{1}\right)\left(Y^{v}\right)=\nabla_{X^{v}}^{L C}\left(\theta_{1}\right)\left(Y^{h}\right)=0 \quad \text { and } \quad \nabla_{X^{v}}^{L C}\left(\theta_{1}\right)\left(Y^{v}\right)=\frac{1}{2} \theta_{0}\left(\gamma_{X}^{\star} Y+\gamma_{Y}^{\star} X\right) .
$$

On the other hand, if (17) holds then $\theta_{0}\left(\gamma_{X}^{\star} Y-\gamma_{Y}^{\star} X\right)=0$. Thus $\left(T M, J_{1}, g_{1}\right)$ is Vaisman if and only if (17) holds, $D \theta_{0}=0$ and, for any $X, Y \in \Gamma(T M), \theta_{0}\left(\gamma_{X}^{\star} Y\right)=0$. This relation is equivalent to $\gamma_{X}(\Pi)=0$ for any $X \in \Gamma(T M)$, $D \theta_{0}=0$ is equivalent to $D \Pi=0$ and we get the desired result since $\gamma=D-\nabla$.

Let us compute the difference tensor $\Gamma=\nabla^{L C}-\nabla^{1}$ of $\left(T M, \nabla^{1}, g_{1}\right)$ as well as its adjoint $\Gamma^{\star}$, the Koszul forms $\alpha_{k}, \xi_{k}$ as well as the Lee form $\theta_{k}$ of $\left(T^{k} M, \nabla^{k}, g_{k}\right)$.

Proposition 3.7. For any $X, Y \in \Gamma(T M)$,

$$
\begin{aligned}
\Gamma_{X^{h}} Y^{h} & =\left(\gamma_{X} Y\right)^{h}, \Gamma_{X^{h}} Y^{v}=\Gamma_{Y^{v}} X^{h}=\frac{1}{2}\left(\gamma_{X} Y+\gamma_{X}^{\star} Y\right)^{v}, \Gamma_{X^{v}} Y^{v}=-\frac{1}{2}\left(\gamma_{X}^{\star} Y+\gamma_{Y}^{\star} X\right)^{h}, \\
\Gamma_{X^{h}}^{\star} Y^{h} & =\left(\gamma_{X}^{\star} Y\right)^{h}, \Gamma_{X^{h}}^{\star} Y^{v}=\frac{1}{2}\left(\gamma_{X} Y+\gamma_{X}^{\star} Y\right)^{v}, \Gamma_{X^{v}}^{\star} Y^{h}=-\frac{1}{2}\left(\gamma_{X} Y+\gamma_{Y}^{\star} X\right)^{v} \quad \text { and } \Gamma_{X^{v}}^{\star} Y^{v}=\frac{1}{2}\left(\gamma_{X}^{\star} Y+\gamma_{Y}^{\star} X\right)^{h}, \\
\operatorname{tr}_{g_{1}}(\Gamma) & =\left(\operatorname{tr}_{\langle,\rangle}(\gamma)-\operatorname{tr}_{\langle,\rangle}\left(\gamma^{\star}\right)\right)^{h}, \operatorname{tr}_{g_{1}}\left(\Gamma^{\star}\right)=2\left(\operatorname{tr}\langle,\rangle\left(\gamma^{\star}\right)\right)^{h}, \\
\xi_{k} & =-\theta_{k}, \alpha_{k}=2^{k} \pi_{k}^{\star} \circ \ldots \circ \pi_{1}^{\star}(\alpha) \quad \text { and } \quad \theta_{k}=\pi_{k}^{\star} \circ \ldots \circ \pi_{1}^{\star}\left(\left(2^{k}-1\right) \alpha-\xi\right), \quad k \geq 1 .
\end{aligned}
$$


Proof. The expressions of $\Gamma$ are an immediate consequence of Proposition 3.3 and (8) and one can deduce easily $\Gamma^{\star}$. If $\left(E_{1}, \ldots, E_{n}\right)$ is a local $\langle$,$\rangle -orthonormal frame then$

$\operatorname{tr}_{g_{1}}(\Gamma)=\sum_{i=1}^{n}\left(\Gamma_{E_{i}^{h}} E_{i}^{h}+\Gamma_{E_{i}^{v}} E_{i}^{v}\right)=\left(\operatorname{tr}_{\langle,\rangle}(\gamma)-\operatorname{tr}_{\langle,\rangle}\left(\gamma^{\star}\right)\right)^{h} \quad$ and $\quad \operatorname{tr}_{g_{1}}\left(\Gamma^{\star}\right)=\sum_{i=1}^{n}\left(\Gamma_{E_{i}^{h}}^{\star} E_{i}^{h}+\Gamma_{E_{i}^{v}}^{\star} E_{i}^{v}\right)=2\left(\operatorname{tr}_{\langle,\rangle}\left(\gamma^{\star}\right)\right)^{h}$.

This implies that $\xi_{1}=\pi_{1}^{\star}(\xi-\alpha)=-\theta_{1}, \alpha_{1}=2 \pi_{1}^{\star}(\alpha)$ and hence

$$
\theta_{2}=\pi_{2}^{\star}\left(\alpha_{1}-\xi_{1}\right)=\pi_{2}^{\star} \circ \pi_{1}(3 \alpha-\xi), \xi_{2}=\pi_{2}^{\star}\left(\xi_{1}-\alpha_{1}\right)=-\theta_{2} \quad \text { and } \quad \alpha_{2}=2 \pi_{2}^{*}\left(\alpha_{1}\right)=2^{2} \pi_{2}^{\star} \circ \pi_{1}(\alpha) .
$$

By induction, we get all the desired formulas.

We end this section by a remark on the Hermitian structure $\left(T M, J_{1}, g_{1}\right)$. Indeed, the fact that $\nabla^{1}\left(J_{1}\right)=0$ makes the Hermitian structure $\left(T M, J_{1}, g_{1}\right)$ particular as the following remark suggests. We don't use this remark in our paper but, may be, it can be used in further studies.

Remark 1. One can check that the tensor $\Gamma$ satisfies, for any $U, V \in \Gamma(T T M)$,

$$
\Gamma_{J_{1} U} J_{1} V-J_{1} \Gamma_{J_{1} U} V-J_{1} \Gamma_{U} J_{1} V-\Gamma_{U} V=0 .
$$

By using the fact that $\nabla^{1}\left(U_{1}\right)=0$ and the known formula (see [18, Proposition 4.2])

$$
4 g_{1}\left(\nabla_{U}^{L C}\left(J_{1}\right) V, W\right)=6 d \omega\left(U, J_{1} V, J_{1} W\right)-6 d \omega(U, V, W)+g_{1}\left(N_{J_{1}}(V, W), J_{1} U\right)
$$

we get that (18) is equivalent to

$$
d \omega\left(J_{1} U, J_{1} V, J_{1} W\right)=d \omega\left(J_{1} U, V, W\right)+d \omega\left(U, J_{1} V, W\right)+d \omega\left(U, V, J_{1} W\right) .
$$

\section{Affine-Riemann manifolds with pluriclosed $\left(T M, J_{1}, g_{1}\right)$}

In this section, we give the conditions so that $\left(T M, J_{1}, g_{1}\right)$ is pluriclosed and we generalize a result obtained in the theory of Hessian manifolds.

Let us compute $d d^{c} \omega=-d J_{1}^{-1} d J_{1} \omega=d J_{1} d \omega$.

Proposition 4.1. For any $X, Y, Z, U \in \Gamma(T M)$,

$$
\left\{\begin{array}{l}
d J_{1} d \omega\left(X^{h}, Y^{h}, Z^{h}, U^{h}\right)=d J_{1} d \omega\left(X^{v}, Y^{v}, Z^{v}, U^{v}\right)=d J_{1} d \omega\left(X^{h}, Y^{h}, Z^{h}, U^{v}\right)=d J_{1} d \omega\left(X^{h}, Y^{v}, Z^{v}, U^{v}\right)=0, \\
d J_{1} d \omega\left(X^{h}, Y^{h}, Z^{v}, U^{v}\right)=2\left\langle K(X, Y) Z-\left(\gamma_{X}^{\star} \circ \gamma_{Y}-\gamma_{Y}^{\star} \circ \gamma_{X}\right) Z, U\right\rangle \circ \pi_{1} .
\end{array}\right.
$$

In particular, $\left(T M, J_{1}, g_{1}\right)$ is pluriclosed if and only if for any $X, Y \in \Gamma(T M)$, the curvature $K$ of $D$ satisfies

$$
K(X, Y)=\gamma_{X}^{\star} \circ \gamma_{Y}-\gamma_{Y}^{\star} \circ \gamma_{X} .
$$

Proof. Put $v(X, Y)=\gamma_{X}^{\star} Y-\gamma_{Y}^{\star} X$. By using Proposition 3.2, we get easily

$$
d J_{1} d \omega\left(X^{h}, Y^{h}, Z^{h}, U^{h}\right)=d J_{1} d \omega\left(X^{v}, Y^{v}, Z^{v}, U^{v}\right)=d J_{1} d \omega\left(X^{h}, Y^{h}, Z^{h}, U^{v}\right)=d J_{1} d \omega\left(X^{h}, Y^{v}, Z^{v}, U^{v}\right)=0 .
$$

On the other hand, having in mind (7), let us compute $S:=d J_{1} d \omega\left(X^{h}, Y^{h}, Z^{v}, U^{v}\right)$. Indeed,

$$
\begin{aligned}
S & =X^{h} . J_{1} d \omega\left(Y^{h}, Z^{v}, U^{v}\right)-Y^{h} . J_{1} d \omega\left(X^{h}, Z^{v}, U^{v}\right)-J_{1} d \omega\left([X, Y]^{h}, Z^{v}, U^{v}\right)+J_{1} d \omega\left(\left(\nabla_{X} Z\right)^{v}, Y^{h}, U^{v}\right) \\
& -J_{1} d \omega\left(\left(\nabla_{X} U\right)^{v}, Y^{h}, Z^{v}\right)-J_{1} d \omega\left(\left(\nabla_{Y} Z\right)^{v}, X^{h}, U^{v}\right)+J_{1} d \omega\left(\left(\nabla_{Y} U\right)^{v}, X^{h}, Z^{v}\right) \\
& =-X .\langle v(Z, U), Y\rangle \circ \pi_{1}+Y .\langle v(Z, U), X\rangle \circ \pi_{1}+\langle v(Z, U),[X, Y]\rangle \circ \pi_{1}+\left\langle v\left(\nabla_{X} Z, U\right), Y\right\rangle \circ \pi_{1} \\
& +\left\langle v\left(Z, \nabla_{X} U\right), Y\right\rangle \circ \pi_{1}-\left\langle v\left(\nabla_{Y} Z, U\right), X\right\rangle \circ \pi_{1}-\left\langle v\left(Z, \nabla_{Y} U\right), X\right\rangle \circ \pi_{1} \\
& =-X .\left\langle\gamma_{Z}^{\star} U, Y\right\rangle \circ \pi_{1}+X .\left\langle\gamma_{U}^{\star} Z, Y\right\rangle \circ \pi_{1}+Y .\left\langle\gamma_{Z}^{\star} U, X\right\rangle \circ \pi_{1}-Y .\left\langle\gamma_{U}^{\star} Z, X\right\rangle \circ \pi_{1}+\left\langle\gamma_{Z}^{\star} U,[X, Y]\right\rangle \circ \pi_{1}
\end{aligned}
$$




$$
\begin{aligned}
& -\left\langle\gamma_{U}^{\star} Z,[X, Y]\right\rangle \circ \pi_{1}+\left\langle\gamma_{D_{X} Z}^{\star} U, Y\right\rangle \circ \pi_{1}-\left\langle\gamma_{U}^{\star} D_{X} Z, Y\right\rangle \circ \pi_{1}-\left\langle\gamma_{\gamma_{X} Z}^{\star} U, Y\right\rangle \circ \pi_{1}+\left\langle\gamma_{U}^{\star} \gamma_{X} Z, Y\right\rangle \circ \pi_{1} \\
& +\left\langle\gamma_{Z}^{\star} D_{X} U, Y\right\rangle \circ \pi_{1}-\left\langle\gamma_{D_{X} U}^{\star} Z, Y\right\rangle \circ \pi_{1}-\left\langle\gamma_{Z}^{\star} \gamma_{X} U, Y\right\rangle \circ \pi_{1}+\left\langle\gamma_{\gamma_{X} U}^{\star} Z, Y\right\rangle \circ \pi_{1} \\
& -\left\langle\gamma_{D_{Y} Z}^{\star} U, X\right\rangle \circ \pi_{1}+\left\langle\gamma_{U}^{\star} D_{Y} Z, X\right\rangle \circ \pi_{1}+\left\langle\gamma_{\gamma_{Y} Z}^{\star} U, X\right\rangle \circ \pi_{1}-\left\langle\gamma_{U}^{\star} \gamma_{Y} Z, X\right\rangle \circ \pi_{1} \\
& -\left\langle\gamma_{Z}^{\star} D_{Y} U, X\right\rangle \circ \pi_{1}+\left\langle\gamma_{D_{Y} U}^{\star} Z, X\right\rangle \circ \pi_{1}+\left\langle\gamma_{Z}^{\star} \gamma_{Y} U, X\right\rangle \circ \pi_{1}-\left\langle\gamma_{\gamma_{Y} U}^{\star} Z, X\right\rangle \circ \pi_{1} .
\end{aligned}
$$

We simplify this expression by using the properties of $D$ :

$$
\begin{aligned}
& S=-\left\langle D_{X} \gamma_{Z}^{\star} U, Y\right\rangle \circ \pi_{1}+\left\langle D_{X} \gamma_{U}^{\star} Z, Y\right\rangle \circ \pi_{1}+\left\langle D_{Y} \gamma_{Z}^{\star} U, X\right\rangle \circ \pi_{1}-\left\langle D_{Y} \gamma_{U}^{\star} Z, X\right\rangle \circ \pi_{1} \\
& +\left\langle\gamma_{D_{X} Z}^{\star} U, Y\right\rangle \circ \pi_{1}-\left\langle\gamma_{U}^{\star} D_{X} Z, Y\right\rangle \circ \pi_{1}-\left\langle\gamma_{\gamma_{X} Z}^{\star} U, Y\right\rangle \circ \pi_{1}+\left\langle\gamma_{U}^{\star} \gamma_{X} Z, Y\right\rangle \circ \pi_{1} \\
& +\left\langle\gamma_{Z}^{\star} D_{X} U, Y\right\rangle \circ \pi_{1}-\left\langle\gamma_{D_{X} U}^{\star} Z, Y\right\rangle \circ \pi_{1}-\left\langle\gamma_{Z}^{\star} \gamma_{X} U, Y\right\rangle \circ \pi_{1}+\left\langle\gamma_{\gamma_{X} U}^{\star} Z, Y\right\rangle \circ \pi_{1} \\
& -\left\langle\gamma_{D_{Y} Z}^{\star} U, X\right\rangle \circ \pi_{1}+\left\langle\gamma_{U}^{\star} D_{Y} Z, X\right\rangle \circ \pi_{1}+\left\langle\gamma_{\gamma_{Y} Z}^{\star} U, X\right\rangle \circ \pi_{1}-\left\langle\gamma_{U}^{\star} \gamma_{Y} Z, X\right\rangle \circ \pi_{1} \\
& -\left\langle\gamma_{Z}^{\star} D_{Y} U, X\right\rangle \circ \pi_{1}+\left\langle\gamma_{D_{Y} U}^{\star} Z, X\right\rangle \circ \pi_{1}+\left\langle\gamma_{Z}^{\star} \gamma_{Y} U, X\right\rangle \circ \pi_{1}-\left\langle\gamma_{\gamma_{Y} U}^{*} Z, X\right\rangle \circ \pi_{1} \\
& =-\left\langle D_{X}\left(\gamma^{\star}\right)(Z, U), Y\right\rangle \circ \pi_{1}+\left\langle D_{X}\left(\gamma^{\star}\right)(U, Z), Y\right\rangle \circ \pi_{1}+\left\langle D_{Y}\left(\gamma^{*}\right)(Z, U), X\right\rangle \circ \pi_{1}-\left\langle D_{Y}\left(\gamma^{\star}\right)(U, Z), X\right\rangle \circ \pi_{1} \\
& -\left\langle\gamma_{\gamma_{X} Z}^{\star} U, Y\right\rangle \circ \pi_{1}+\left\langle\gamma_{U}^{\star} \gamma_{X} Z, Y\right\rangle \circ \pi_{1}-\left\langle\gamma_{Z}^{\star} \gamma_{X} U, Y\right\rangle \circ \pi_{1}+\left\langle\gamma_{\gamma_{X} U}^{\star} Z, Y\right\rangle \circ \pi_{1} \\
& +\left\langle\gamma_{\gamma_{Y} Z}^{\star} U, X\right\rangle \circ \pi_{1}-\left\langle\gamma_{U}^{\star} \gamma_{Y} Z, X\right\rangle \circ \pi_{1}+\left\langle\gamma_{Z}^{\star} \gamma_{Y} U, X\right\rangle \circ \pi_{1}-\left\langle\gamma_{\gamma_{Y} U}^{\star} Z, X\right\rangle \circ \pi_{1} .
\end{aligned}
$$

By using (12), we get

$$
\begin{aligned}
S & =-\left\langle D_{X}(\gamma)(Z, Y), U\right\rangle \circ \pi_{1}+\left\langle D_{X}(\gamma)(U, Y), Z\right\rangle \circ \pi_{1}+\left\langle D_{Y}(\gamma)(Z, X), U\right\rangle \circ \pi_{1}-\left\langle D_{Y}(\gamma)(U, X), Z\right\rangle \circ \pi_{1} \\
& -\left\langle U, \gamma_{Y} \circ \gamma_{X} Z\right\rangle \circ \pi_{1}+\left\langle\gamma_{Y}^{\star} \circ \gamma_{X} Z, U\right\rangle \circ \pi_{1}-\left\langle U, \gamma_{X}^{\star} \circ \gamma_{Y} Z\right\rangle \circ \pi_{1}+\left\langle Z, \gamma_{Y} \circ \gamma_{X} U\right\rangle \circ \pi_{1} \\
& +\left\langle U, \gamma_{X} \circ \gamma_{Y} Z\right\rangle \circ \pi_{1}-\left\langle\gamma_{X}^{\star} \circ \gamma_{Y} Z, U\right\rangle \circ \pi_{1}+\left\langle U, \gamma_{Y}^{\star} \circ \gamma_{X} Z\right\rangle \circ \pi_{1}-\left\langle\gamma_{Y}^{\star} \circ \gamma_{X}^{\star} Z, U\right\rangle \circ \pi_{1} \\
& \stackrel{(13)}{=}\langle K(X, Y) Z, U\rangle \circ \pi_{1}-\langle K(X, Y) U, Z\rangle \circ \pi_{1}+2\left\langle\left(\gamma_{Y}^{\star} \circ \gamma_{X}-\gamma_{X}^{\star} \circ \gamma_{Y}\right) Z, U\right\rangle \circ \pi_{1} \\
& =2\left\langle K(X, Y) Z-\left(\gamma_{X}^{\star} \circ \gamma_{Y}-\gamma_{Y}^{\star} \circ \gamma_{X}\right), U\right\rangle \circ \pi_{1} .
\end{aligned}
$$

In [23, Theorem $8.8 \mathrm{pp} .162]$, Shima proved that if $(M, \nabla,\langle\rangle$,$) is a compact Hessian manifold such that its$ first Koszul form vanishes then $\nabla$ is the Levi-Civita connection of $\langle$,$\rangle . Note that in this case the first Koszul$ form and the dual Koszul form coincide. The following theorem is a generalization of this result under an additional assumption, namely, $\nabla$ is complete. It will be interesting to see if we can drop this assumption.

Theorem 4.1. Let $(M, \nabla,\langle\rangle$,$) be an affine-Riemann manifold such that \left(T M, J_{1}, g_{1}\right)$ is pluriclosed and the dual Koszul form of $(M, \nabla,\langle\rangle$,$) vanishes. Then the Ricci curvature of \langle$,$\rangle is nonnegative. Moreover, if M$ is compact and $\nabla$ is complete then $\gamma=0$, i.e., $\nabla$ is the Levi-Civita connection of $\langle$,$\rangle .$

Proof. Note that the vanishing of dual Koszul form is equivalent to $\operatorname{tr}_{\langle,\rangle}(\gamma)=0$. From the relation

$$
K(X, Y)=\gamma_{X}^{\star} \circ \gamma_{Y}-\gamma_{Y}^{\star} \circ \gamma_{X}
$$

and the fact that $\operatorname{tr}_{\langle,\rangle}(\gamma)=0$, we deduce that the Ricci curvature of $\langle$,$\rangle is given by$

$$
\operatorname{ric}(X, X)=\operatorname{tr}\left(\gamma_{X}^{\star} \circ \gamma_{X}\right) \geq 0
$$

and $\operatorname{ric}(X, X)=0$ if and only if $\gamma_{X}=0$. By using the splitting theorem of J. Cheeger and D. Gromoll (see for instance [5, Corollary 6.67 pp. 168]), we deduce that if $M$ is compact its universal Riemannian covering is isometric to a Riemannian product $\left(\bar{M} \times \mathbb{R}^{d},\langle,\rangle_{1} \times\langle,\rangle_{0}\right)$ where $\bar{M}$ is compact and $\langle,\rangle_{0}$ is the canonical metric of $\mathbb{R}^{d}$. But if $\nabla$ is complete the universal covering of $M$ is diffeomorphic to $\mathbb{R}^{n}$ which completes the proof. 


\section{The Bismut and Chern connections of $\left(T M, J_{1}, g_{1}\right)$ and their curvatures}

Let $(M, \nabla,\langle\rangle$,$) be an affine-Riemann manifold of dimension n$. The expressions of the Bismut and Chern connections of $\left(T M, J_{1}, g_{1}\right)$ can be deduced easily from (1) and Propositions 3.2-3.3.

Proposition 5.1. We have, for any $X, Y \in \Gamma(T M)$,

$$
\left\{\begin{array} { l } 
{ \nabla _ { X ^ { h } } ^ { B } Y ^ { h } = ( D _ { X } Y ) ^ { h } , \nabla _ { X ^ { v } } ^ { B } Y ^ { v } = - ( \gamma _ { Y } ^ { \star } X ) ^ { h } , } \\
{ \nabla _ { X ^ { v } } ^ { B } Y ^ { h } = ( \gamma _ { Y } ^ { \star } X ) ^ { v } , \nabla _ { X ^ { h } } ^ { B } Y ^ { v } = ( D _ { X } Y ) ^ { v } . }
\end{array} \quad \text { and } \quad \left\{\begin{array}{l}
\nabla_{X^{h}}^{C} Y^{h}=\left(D_{X} Y\right)^{h}-\left(\gamma_{X}^{a} Y\right)^{h}, \nabla_{X^{v}}^{C} Y^{v}=-\left(\gamma_{X}^{s} Y\right)^{h}, \\
\nabla_{X^{v}}^{C} Y^{h}=\left(\gamma_{X}^{s} Y\right)^{v}, \nabla_{X^{h}}^{C} Y^{v}=\left(D_{X} Y\right)^{v}-\left(\gamma_{X}^{a} Y\right)^{v} .
\end{array}\right.\right.
$$

where

$$
\gamma^{a}=\frac{1}{2}\left(\gamma-\gamma^{\star}\right) \quad \text { and } \quad \gamma^{s}=\frac{1}{2}\left(\gamma+\gamma^{\star}\right) .
$$

Now, we give the curvature $R^{B}$ of $\nabla^{B}$.

Proposition 5.2. For any $X, Y, Z \in \Gamma(T M)$,

$$
\left\{\begin{array}{l}
R^{B}\left(X^{h}, Y^{h}\right) Z^{h}=(K(X, Y) Z)^{h}, R^{B}\left(X^{h}, Y^{h}\right) Z^{v}=(K(X, Y) Z)^{v}, \\
R^{B}\left(X^{v}, Y^{v}\right) Z^{v}=\left(\gamma_{\gamma_{Z}^{*} Y}^{*} X\right)^{v}-\left(\gamma_{\gamma_{Z}^{*} X}^{*} Y\right)^{v}, R^{B}\left(X^{v}, Y^{v}\right) Z^{h}=\left(\gamma_{\gamma_{Z}^{*} Y}^{*} X\right)^{h}-\left(\gamma_{\gamma_{Z}^{*} X}^{*} Y\right)^{h}, \\
R^{B}\left(X^{h}, Y^{v}\right) Z^{v}=\left(\gamma_{Z}^{*} \circ \gamma_{X} Y\right)^{h}+\left(D_{X}\left(\gamma^{*}\right)(Z, Y)\right)^{h}, \\
\left.R^{B}\left(X^{h}, Y^{v}\right) Z^{h}=-\left(\gamma_{Z}^{*} \circ \gamma_{X} Y\right)\right)^{v}-\left(D_{X}\left(\gamma^{*}\right)(Z, Y)\right)^{v},
\end{array}\right.
$$

where $K$ is the curvature of D. Moreover, the Ricci form is given by

$$
\rho^{B}\left(X^{h}, Y^{h}\right)=\rho^{B}\left(X^{\nu}, Y^{\nu}\right)=0 \quad \text { and } \rho^{B}\left(X^{h}, Y^{\nu}\right)=-\left\langle\gamma_{X} Y, \operatorname{tr}_{\langle,\rangle} \gamma\right\rangle \circ \pi_{1}-\left\langle D_{X} \operatorname{tr}_{\langle,\rangle}(\gamma), Y\right\rangle \circ \pi_{1} .
$$

In particular, if $\operatorname{tr}_{\langle,\rangle}(\gamma)=0$ then $\left(T M, J_{1}, g_{1}\right)$ is Calabi-Yau with torsion, i.e., $\rho^{B}=0$.

Proof. We have

$$
\begin{aligned}
R^{B}\left(X^{h}, Y^{h}\right) Z^{h} & =(K(X, Y) Z)^{h} \\
R^{B}\left(X^{h}, Y^{h}\right) Z^{v} & =(K(X, Y) Z)^{v}, \\
R^{B}\left(X^{v}, Y^{v}\right) Z^{v} & =\nabla_{X^{v}}^{B}\left(\gamma_{Z}^{\star} Y\right)^{h}-\nabla_{Y^{v}}^{B}\left(\gamma_{Z}^{\star} X\right)^{h}=\left(\gamma_{\gamma_{Z}^{*} Y}^{*} X\right)^{v}-\left(\gamma_{\gamma_{Z}^{*} X}^{\star} Y\right)^{v}, \\
R^{B}\left(X^{v}, Y^{v}\right) Z^{h} & =-\nabla_{X^{v}}^{B}\left(\gamma_{Z}^{\star} Y\right)^{v}+\nabla_{Y^{v}}^{B}\left(\gamma_{Z}^{*} X\right)^{v}=\left(\gamma_{\gamma_{Z}^{*} Y}^{\star} X\right)^{h}-\left(\gamma_{\gamma_{Z}^{*} X}^{\star} Y\right)^{h}, \\
R^{B}\left(X^{h}, Y^{v}\right) Z^{v} & =\nabla_{\left(\nabla_{X} Y\right)^{v}}^{B} Z^{v}+\nabla_{X^{h}}^{B}\left(\gamma_{Z}^{\star} Y\right)^{h}+\nabla_{Y^{v}}^{B}\left(D_{X} Z\right)^{v} \\
& =-\left(\gamma_{Z}^{\star}\left(\nabla_{X} Y\right)\right)^{h}+\left(D_{X} \gamma_{Z}^{\star} Y\right)^{h}-\left(\gamma_{D_{X} Z}^{\star} Y\right)^{h}, \\
& =\left(\gamma_{Z}^{\star} \circ \gamma_{X} Y\right)^{h}+\left(D_{X} \gamma_{Z}^{\star} Y\right)^{h}-\left(\gamma_{D_{X} Z}^{\star} Y\right)^{h}-\left(\gamma_{Z}^{\star}\left(D_{X} Y\right)\right)^{h}, \\
R^{B}\left(X^{h}, Y^{v}\right) Z^{h} & =\nabla_{\left(\nabla_{X} Y\right)^{v}}^{B} Z^{h}-\nabla_{X^{h}}^{B}\left(\gamma_{Z}^{\star} Y\right)^{v}+\nabla_{Y^{v}}^{B}\left(D_{X} Z\right)^{h} \\
& =\left(\gamma_{Z}^{\star}\left(\nabla_{X} Y\right)\right)^{v}-\left(D_{X} \gamma_{Z}^{\star} Y\right)^{v}+\left(\gamma_{D_{X} Z}^{\star} Y\right)^{v} \\
& =-\left(\gamma_{Z}^{\star}\left(\gamma_{X} Y\right)\right)^{v}+\left(\gamma_{Z}^{\star}\left(D_{X} Y\right)\right)^{v}-\left(D_{X} \gamma_{Z}^{\star} Y\right)^{v}+\left(\gamma_{D_{X} Z}^{\star} Y\right)^{v} .
\end{aligned}
$$

Let $\left(E_{1}, \ldots, E_{n}\right)$ be a local orthonormal frame of $\langle$,$\rangle . Then$

$$
\begin{aligned}
& 2 \rho^{B}\left(X^{h}, Y^{h}\right)=\sum_{i=1}^{n}\left(g_{1}\left(R^{B}\left(X^{h}, Y^{h}\right) E_{i}^{h}, E_{i}^{v}\right)-g_{1}\left(R^{B}\left(X^{h}, Y^{h}\right) E_{i}^{v}, E_{i}^{h}\right)\right)=0, \\
& 2 \rho^{B}\left(X^{v}, Y^{v}\right)=\sum_{i=1}^{n}\left(g_{1}\left(R^{B}\left(X^{v}, Y^{v}\right) E_{i}^{h}, E_{i}^{v}\right)-g_{1}\left(R^{B}\left(X^{v}, Y^{v}\right) E_{i}^{v}, E_{i}^{h}\right)\right)=0,
\end{aligned}
$$




$$
\begin{aligned}
2 \rho^{B}\left(X^{h}, Y^{v}\right) & =\sum_{i=1}^{n}\left(g_{1}\left(R^{B}\left(X^{h}, Y^{v}\right) E_{i}^{h}, E_{i}^{v}\right)-g_{1}\left(R^{B}\left(X^{h}, Y^{v}\right) E_{i}^{v}, E_{i}^{h}\right)\right) \\
& =\sum_{i=1}^{n}\left(-2\left\langle\gamma_{E_{i}}^{\star} \circ \gamma_{X} Y+D_{X}\left(\gamma^{*}\right)\left(E_{i}, Y\right), E_{i}\right\rangle\right) \\
& =-2\left\langle\gamma_{X} Y, \operatorname{tr}_{\langle,}, \gamma\right\rangle \circ \pi_{1}-2 \sum_{i=1}^{n}\left\langle D_{X}\left(\gamma^{*}\right)\left(E_{i}, Y\right), E_{i}\right\rangle \circ \pi_{1} \\
& \stackrel{(12)}{=}-2\left\langle\gamma_{X} Y, \operatorname{tr} \gamma\right\rangle \circ \pi_{1}-2 \sum_{i=1}^{n}\left\langle D_{X}(\gamma)\left(E_{i}, E_{i}\right), Y\right\rangle \circ \pi_{1} \\
& =-2\left\langle\gamma_{X} Y, \operatorname{tr}_{\langle,\rangle}, \gamma\right\rangle \circ \pi_{1}-2\left\langle\operatorname{tr}_{\langle,\rangle}\left(D_{X}(\gamma)\right), Y\right\rangle \circ \pi_{1} .
\end{aligned}
$$

Fix a point $p \in M$. It is known that there exists a local orthonormal frame $\left(E_{1}, \ldots, E_{n}\right)$ in a neighborhood of $p$ such that $\left(D E_{j}\right)(p)=0$ for $j=1, \ldots, n$. For any $X \in \Gamma(T M)$,

$$
\begin{aligned}
\operatorname{tr}_{\langle,\rangle}\left(D_{X}(\gamma)\right) & =\sum_{i=1}^{n} D_{X}(\gamma)\left(E_{i}, E_{i}\right) \\
& =D_{X}\left(\operatorname{tr}_{\langle,\rangle}(\gamma)\right)-2 \gamma_{D_{X} E_{i}} E_{i}
\end{aligned}
$$

By evaluating at $p$ we get that $\operatorname{tr}_{\langle,\rangle}\left(D_{X}(\gamma)\right)(p)=D_{X}\left(\operatorname{tr}_{\langle,\rangle}(\gamma)\right)(p)$ which completes the proof.

We give also the curvature $R^{C}$ of $\nabla^{C}$.

Proposition 5.3. For any $X, Y, Z \in \Gamma(T M)$,

$$
\left\{\begin{array}{l}
R^{C}\left(X^{h}, Y^{h}\right) Z^{h}=R^{C}\left(X^{v}, Y^{v}\right) Z^{h}=\left(\left[\gamma_{X}^{s}, \gamma_{Y}^{s}\right] Z\right)^{h}, \\
R^{C}\left(X^{h}, Y^{h}\right) Z^{v}=R^{C}\left(X^{v}, Y^{v}\right) Z^{v}=\left(\left[\gamma_{X}^{s}, \gamma_{Y}^{s}\right] Z\right)^{v}, \\
R^{C}\left(X^{h}, Y^{v}\right) Z^{h}=-\left(D_{X}\left(\gamma^{s}\right)(Y, Z)\right)^{v}+\left(\left[\gamma_{X}^{a}, \gamma_{Y}^{s}\right] Z\right)^{v}-\left(\gamma_{\gamma_{X} Y}^{s} Z\right)^{v}, \\
R^{C}\left(X^{h}, Y^{v}\right) Z^{v}=\left(D_{X}\left(\gamma^{s}\right)(Y, Z)\right)^{h}-\left(\left[\gamma_{X}^{a}, \gamma_{Y}^{s}\right] Z\right)^{h}+\left(\gamma_{\gamma_{X} Y}^{s} Z\right)^{h},
\end{array}\right.
$$

where $K$ is the curvature of $D$. Moreover, the Ricci form is given by

$$
\rho^{C}\left(X^{h}, Y^{h}\right)=\rho^{C}\left(X^{v}, Y^{v}\right)=0 \quad \text { and } \quad \rho^{C}\left(X^{h}, Y^{v}\right)=-\left\langle\gamma_{X} Y, \operatorname{tr}_{\langle,\rangle}\left(\gamma^{\star}\right)\right\rangle \circ \pi_{1}-\left\langle D_{X} \operatorname{tr}_{\langle,\rangle}\left(\gamma^{\star}\right), Y\right\rangle \circ \pi_{1} \text {. }
$$

In particular, if $\operatorname{tr}_{\langle,\rangle}\left(\gamma^{*}\right)=0$ then the Ricci form $\rho^{C}$ of $\left(T M, J_{1}, g_{1}\right)$ vanishes.

Proof. By using Proposition 5.1,

$$
\begin{gathered}
R^{C}\left(X^{h}, Y^{h}\right) Z^{h}=\left(D_{[X, Y]} Z\right)^{h}-\left(\gamma_{[X, Y]}^{a} Z\right)^{h}-\nabla_{X^{h}}^{C}\left(D_{Y} Z\right)^{h}+\nabla_{X^{h}}^{C}\left(\gamma_{Y}^{a} Z\right)^{h}+\nabla_{Y^{h}}^{C}\left(D_{X} Z\right)^{h}-\nabla_{Y^{h}}^{C}\left(\gamma_{X}^{a} Z\right)^{h} \\
=\left(D_{[X, Y]} Z\right)^{h}-\left(\gamma_{[X, Y]}^{a} Z\right)^{h}-\left(D_{X} D_{Y} Z\right)^{h}+\left(\gamma_{X}^{a} D_{Y} Z\right)^{h}+\left(D_{X} \gamma_{Y}^{a} Z\right)^{h}-\left(\gamma_{X}^{a} \gamma_{Y}^{a} Z\right)^{h} \\
+\left(D_{Y} D_{X} Z\right)^{h}-\left(\gamma_{Y}^{a} D_{X} Z\right)^{h}-\left(D_{Y} \gamma_{X}^{a} Z\right)^{h}+\left(\gamma_{Y}^{a} \gamma_{X}^{a} Z\right)^{h} \\
=(K(X, Y) Z)^{h}+\left(D_{X}\left(\gamma^{a}\right)(Y, Z)\right)^{h}-\left(D_{Y}\left(\gamma^{a}\right)(X, Z)\right)^{h}+\left(\left[\gamma_{Y}^{a}, \gamma_{X}^{a}\right] Z\right)^{h} . \\
R^{C}\left(X^{v}, Y^{v}\right) Z^{v}=\nabla_{X^{v}}^{C}\left(\gamma_{Y}^{s} Z\right)^{h}-\nabla_{Y^{v}}^{C}\left(\gamma_{X}^{s} Z\right)^{h} \\
=\left(\gamma_{X}^{s} \gamma_{Y}^{s} Z\right)^{v}-\left(\gamma_{Y}^{s} \gamma_{X}^{s} Z\right)^{v}=\left(\left[\gamma_{X}^{s}, \gamma_{Y}^{s}\right] Z\right)^{v} \\
R^{C}\left(X^{v}, Y^{v}\right) Z^{h}=-\nabla_{X^{v}}^{C}\left(\gamma_{Y}^{s} Z\right)^{v}+\nabla_{Y^{v}}^{C}\left(\gamma_{X}^{s} Z\right)^{v} \\
=\left(\left[\gamma_{X}^{s}, \gamma_{Y}^{s}\right] Z\right)^{h} . \\
R^{C}\left(X^{h}, Y^{v}\right) Z^{h}=\nabla_{\left(\nabla_{X} Y\right)^{v}}^{C} Z^{h}-\nabla_{X^{h}}^{C}\left(\gamma_{Y}^{s} Z\right)^{v}+\nabla_{Y^{v}}^{C}\left(D_{X} Z\right)^{h}-\nabla_{Y^{v}}^{C}\left(\gamma_{X}^{a} Z\right)^{h}
\end{gathered}
$$




$$
\begin{aligned}
& =\left(\gamma_{D_{X} Y}^{s} Z\right)^{v}-\left(\gamma_{\gamma_{X} Y}^{s} Z\right)^{v}-\left(D_{X} \gamma_{Y}^{s} Z\right)^{v}+\left(\gamma_{X}^{a} \gamma_{Y}^{s} Z\right)^{v}+\left(\gamma_{Y}^{s} D_{X} Z\right)^{v}-\left(\gamma_{Y}^{s} \gamma_{X}^{a} Z\right)^{v} \\
& =-\left(D_{X}\left(\gamma^{s}\right)(Y, Z)\right)^{v}+\left(\left[\gamma_{X}^{a}, \gamma_{Y}^{s}\right] Z\right)^{v}-\left(\gamma_{\gamma_{X} Y}^{s} Z\right)^{v}, \\
R^{C}\left(X^{h}, Y^{v}\right) Z^{v} & =\nabla_{\left(\nabla_{X} Y\right)^{v}}^{C} Z^{v}+\nabla_{X^{h}}^{C}\left(\gamma_{Y}^{s} Z\right)^{h}+\nabla_{Y^{v}}^{C}\left(D_{X} Z\right)^{v}-\nabla_{Y^{v}}^{C}\left(\gamma_{X}^{a} Z\right)^{v} \\
& =\left(\gamma_{\gamma_{X} Y}^{s} Z\right)^{h}-\left(\gamma_{D_{X} Y}^{s} Z\right)^{h}+\left(D_{X} \gamma_{Y}^{s} Z\right)^{h}-\left(\gamma_{X}^{a} \gamma_{Y}^{s} Z\right)^{h}-\left(\gamma_{Y}^{s} D_{X} Z\right)^{h}+\left(\gamma_{Y}^{s} \gamma_{X}^{a} Z\right)^{h}, \\
& =\left(D_{X}\left(\gamma^{s}\right)(Y, Z)\right)^{v}-\left(\left[\gamma_{X}^{a}, \gamma_{Y}^{s}\right] Z\right)^{v}+\left(\gamma_{\gamma_{X} Y}^{s} Z\right)^{v},
\end{aligned}
$$

Now, by using (14) and (15)

$$
\begin{aligned}
R^{C}\left(X^{h}, Y^{h}\right) Z^{h} & =\left(D_{Y}(\gamma)(X, Z)\right)^{h}-\left(D_{X}(\gamma)(Y, Z)\right)^{h}+\left(\left[\gamma_{X}, \gamma_{Y}\right] Z\right)^{h}++\left(D_{X}\left(\gamma^{a}\right)(Y, Z)\right)^{h}-\left(D_{Y}\left(\gamma^{a}\right)(X, Z)\right)^{h}+\left(\left[\gamma_{Y}^{a}, \gamma_{X}^{a}\right] Z\right)^{h} \\
& =\frac{1}{2} D_{Y}\left(\gamma+\gamma^{\star}\right)(X, Z)-\frac{1}{2} D_{X}\left(\gamma+\gamma^{\star}\right)(Y, Z)+\left(\left[\gamma_{X}, \gamma_{Y}\right] Z\right)^{h}+\left(\left[\gamma_{Y}^{a}, \gamma_{X}^{a}\right] Z\right)^{h} \\
& =\frac{1}{2}\left(\left[\gamma_{X}^{\star}, \gamma_{Y}^{\star}\right] Z\right)^{h}-\frac{1}{2}\left(\left[\gamma_{X}, \gamma_{Y}\right] Z\right)^{h}+\left(\left[\gamma_{X}, \gamma_{Y}\right] Z\right)^{h}+\frac{1}{4}\left(\left[\gamma_{Y}-\gamma_{Y}^{\star}, \gamma_{X}-\gamma_{X}^{\star}\right] Z\right)^{h} \\
& =\frac{1}{2}\left(\left[\gamma_{X}^{\star}, \gamma_{Y}^{\star}\right] Z\right)^{h}+\frac{1}{2}\left(\left[\gamma_{X}, \gamma_{Y}\right] Z\right)^{h}+\frac{1}{4}\left(\left[\gamma_{Y}-\gamma_{Y}^{\star}, \gamma_{X}-\gamma_{X}^{\star}\right] Z\right)^{h} \\
& =\frac{1}{4}\left(\left[\gamma_{X}^{\star}, \gamma_{Y}^{\star}\right] Z\right)^{h}+\frac{1}{4}\left(\left[\gamma_{X}, \gamma_{Y}\right] Z\right)^{h}-\frac{1}{4}\left(\left[\gamma_{Y}, \gamma_{X}^{\star}\right] Z+\left[\gamma_{Y}^{\star}, \gamma_{X}\right] Z\right)^{h} \\
& =\left[\gamma_{X}^{s}, \gamma_{Y}^{s}\right] .
\end{aligned}
$$

Let $\left(E_{1}, \ldots, E_{n}\right)$ be a local orthonormal frame of $\langle$,$\rangle . We have obviously, \rho^{C}\left(X^{h}, Y^{h}\right)=\rho^{C}\left(X^{v}, Y^{v}\right)=0$. Then

$$
\begin{aligned}
2 \rho^{C}\left(X^{h}, Y^{v}\right) & =\sum_{i=1}^{n}\left(g_{1}\left(R^{C}\left(X^{h}, Y^{v}\right) E_{i}^{h}, J E_{i}^{h}\right)+g_{1}\left(R^{C}\left(X^{h}, Y^{v}\right) E_{i}^{v}, J E_{i}^{v}\right)\right) \\
& =\sum_{i=1}^{n}\left(g_{1}\left(R^{C}\left(X^{h}, Y^{v}\right) E_{i}^{h}, E_{i}^{v}\right)-g_{1}\left(R^{C}\left(X^{h}, Y^{v}\right) E_{i}^{v}, E_{i}^{h}\right)\right) \\
& =2 \sum_{i=1}^{n}\left(\left\langle-D_{X}\left(\gamma^{s}\right)\left(Y, E_{i}\right)+\left[\gamma_{X}^{a}, \gamma_{Y}^{s}\right] E_{i}-\gamma_{\gamma_{X}}^{s} E_{i}, E_{i}\right\rangle \circ \pi_{1}\right) \\
& =-2 \operatorname{tr}\left(\gamma_{\gamma_{X} Y}\right) \circ \pi_{1}-\sum_{i=1}^{n}\left\langle D_{X}(\gamma)\left(Y, E_{i}\right), E_{i}\right\rangle \circ \pi_{1}-\sum_{i=1}^{n}\left\langle D_{X}\left(\gamma^{\star}\right)\left(Y, E_{i}\right), E_{i}\right\rangle \circ \pi_{1} \\
& \stackrel{(16)-(12)}{=}-2\left\langle\gamma_{X} Y, \operatorname{tr}_{\langle,\rangle}\left(\gamma^{\star}\right)\right\rangle \circ \pi_{1}-2\left\langle\operatorname{tr}_{\langle,\rangle}\left(D_{X}\left(\gamma^{\star}\right)\right), Y\right\rangle \circ \pi_{1} .
\end{aligned}
$$

The same argument as at the end of the proof of Proposition 5.2 completes the proof.

\section{The canonical sequence of Hermitian structures of an affine-Riemann manifold: global and local results}

In this section, we prove a global result on the sequence $\left(T^{k} M, J_{k}, g_{k}\right)$, we give in local affine coordinates the necessary and sufficient conditions for $\left(T M, J_{1}, g_{1}\right)$ to be balanced or pluriclosed. We illustrate these results by many examples and we give some properties of affine-Riemann manifolds for which $\left(T M, J_{1}, g_{1}\right)$ is Vaisman.

Let us start with the following result which constitutes one of the main results of this paper.

Theorem 6.1. Let $(M, \nabla,\langle\rangle$,$) be an affine-Riemann manifold. Then:$

1. If $\gamma=0$ then, for any $k \geq 1, \nabla^{k}$ is the Levi-Civita connection of $g_{k}$ and $\left(T^{k} M, J_{k}, g_{k}\right)$ is Kähler flat.

2. For some $k \geq 2,\left(T^{k} M, J_{k}, g_{k}\right)$ is Kähler if and only if $\gamma=0$.

3. For some $k \geq 1,\left(T^{k} M, J_{k}, g_{k}\right)$ is locally conformally balanced if and only if $\left(T M, J_{1}, g_{1}\right)$ is locally conformally balanced and this is equivalent to $d \xi=0$. 
4. For $k_{0} \geq 1,\left(T^{k_{0}} M, J_{k_{0}}, g_{k_{0}}\right)$ is balanced if and only if

$$
\operatorname{tr}_{\langle,\rangle}(\gamma)=\left(2^{k_{0}}-1\right) \operatorname{tr}_{\langle,\rangle}\left(\gamma^{*}\right) .
$$

In this case, $\left(T^{k_{0}+1} M, J_{k_{0}+1}, g_{k_{0}+1}\right)$ is Calabi-Yau with torsion and for any $k \neq k_{0},\left(T^{k} M, J_{k}, g_{k}\right)$ is locally conformally balanced.

5. If $\operatorname{tr}_{\langle,\rangle}(\gamma)=\operatorname{tr}_{\langle,\rangle}\left(\gamma^{*}\right)=0$ then, for any $k \geq 1,\left(T^{k} M, J_{k}, g_{k}\right)$ is balanced, Calabi-Yau with torsion and its Chern Ricci form vanishes.

Proof. Note first that $\gamma=0$ if and only if $D=\nabla$. On the other hand, it is obvious from Proposition 3.7 that $\gamma=0$ if and only if the operator difference $\Gamma$ for $\left(T M, \nabla^{1}, g_{1}\right)$ vanishes. By induction, we get that $\gamma=0$ if and only if, for any $k \geq 1$, the operator difference $\Gamma^{k}$ of $\left(T^{k} M, \nabla^{k}, g_{k}\right)$ vanishes and the result follows.

2. According to Corollary 3.1, $\left(T^{2} M, J_{2}, g_{2}\right)$ is Kähler if and only if $\Gamma^{\star}=\Gamma$. But from Proposition 3.7, this implies that for any $X, Y \in \Gamma(T M)$

$$
\Gamma_{X^{h}} Y^{h}=\left(\gamma_{X} Y\right)^{h}=\Gamma_{X^{h}}^{\star} Y^{h}=\left(\gamma_{X}^{\star} Y\right)^{h} \quad \text { and } \quad \Gamma_{X^{v}} Y^{v}=-\frac{1}{2}\left(\gamma_{X}^{\star} Y+\gamma_{Y}^{\star} X\right)^{h}=\Gamma_{X^{v}}^{\star} Y^{v}=\frac{1}{2}\left(\gamma_{X}^{\star} Y+\gamma_{Y}^{\star} X\right)^{h}
$$

and hence $\gamma=0$. By induction, we get the result.

3. Fix $k \geq 1$. Then $\left(T^{k} M, J_{k}, g_{k}\right)$ is locally conformally balanced if and only if its Lee form $\theta_{k}$ is closed. But from Proposition 3.7, $\theta_{k}=\pi_{k}^{\star} \circ \ldots \circ \pi_{1}^{\star}\left(\left(2^{k}-1\right) \alpha-\xi\right)$. The first Koszul form $\alpha$ being closed we get the result.

4. Fix $k_{0} \geq 1$. Then $\left(T^{k_{0}} M, J_{k_{0}}, g_{k_{0}}\right)$ is balanced if and only if $\theta_{k_{0}}=\pi_{k_{0}}^{\star} \circ \ldots \circ \pi_{1}^{\star}\left(\left(2^{k_{0}}-1\right) \alpha-\xi\right)=0$ which is equivalent to $\operatorname{tr}_{\langle,\rangle}(\gamma)=\left(2^{k_{0}}-1\right) \operatorname{tr}_{\langle,\rangle}\left(\gamma^{\star}\right)$. But from Proposition 3.7, $\theta_{k}=-\xi_{k}$ and one can use Proposition 5.2 to deduce that if $\left(T^{k_{0}} M, J_{k_{0}}, g_{k_{0}}\right)$ is balanced then $\left(T^{k_{0}+1} M, J_{k_{0}+1}, g_{k_{0}+1}\right)$ is Calabi-Yau with torsion. On the other hand, since $\alpha$ is closed then $d \xi=0$ which completes the proof.

5. It is a consequence of what above and Propositions 5.2-5.3.

Example 1. 1. In the item 4 of Theorem 6.1, one can build a balanced Hermitian structure on $T^{k} M$ by solving an equation on $(M, \nabla,\langle\rangle$,$) . Let us give some examples of this situation.$

(a) We consider the left symmetric product on $\mathbb{R}^{3}$ given by

$$
e_{1} \bullet e_{1}=a e_{1}, e_{1} \bullet e_{2}=a e_{2}+e_{3}, e_{1} \bullet e_{3}=e_{2}+a e_{3}, e_{2} \bullet e_{1}=a e_{2}, e_{3} \bullet e_{1}=a e_{3} .
$$

The associated non vanishing Lie brackets are given by

$$
\left[e_{1}, e_{2}\right]=e_{3},\left[e_{1}, e_{3}\right]=e_{2} .
$$

We denote by $G$ the connected simply-connected Lie group associated to $\left(\mathbb{R}^{3},[],\right)$ and by $\nabla$ the left invariant flat torsionless connection on $G$ defined by $\bullet$. For $a=1$, the left invariant metric on $G$ associated to the scalar product $\left[\begin{array}{lll}1 & 1 & 0 \\ 1 & 3 & 1 \\ 0 & 1 & 1\end{array}\right]$ on $\mathbb{R}^{3}$ satisfies (19) for $k_{0}=2$ with $\operatorname{tr}_{\langle,\rangle}(\gamma) \neq 0$ and $\operatorname{tr}_{\langle,\rangle}\left(\gamma^{\star}\right) \neq 0$.

Thus $\left(T^{2} G, J_{2}, g_{2}\right)$ is balanced, $\left(T^{3} G, J_{3}, g_{3}\right)$ is Calabi-Yau with torsion and, for any $k \neq 2,\left(T^{k} G, J_{k}, g_{k}\right)$ is locally conformally balanced not balanced.

(b) We consider the left symmetric product on $\mathbb{R}^{3}$ given by

$$
e_{1} \bullet e_{1}=a e_{1}, e_{1} \bullet e_{2}=(1+a) e_{2}, e_{1} \bullet e_{3}=(1+a) e_{3}, e_{2} \bullet e_{1}=a e_{2}, e_{3} \bullet e_{1}=a e_{3} .
$$

The associated non vanishing Lie brackets are given by

$$
\left[e_{1}, e_{2}\right]=e_{2},\left[e_{1}, e_{3}\right]=e_{3} .
$$

We denote by $G$ the connected simply-connected Lie group associated to $\left(\mathbb{R}^{3},[],\right)$ and by $\nabla$ the left invariant flat torsionless connection on $G$ defined by $\bullet$. For $a=\frac{8}{3}$, the left invariant metric on $G$ associated 
to the scalar product $\left[\begin{array}{ccc}\lambda & 0 & 0 \\ 0 & \mu & 0 \\ 0 & 0 & v\end{array}\right]$ on $\mathbb{R}^{3}$ satisfies (19) for $k_{0}=4$ with $\operatorname{tr}_{\langle,\rangle}(\gamma) \neq 0$ and $\operatorname{tr}_{\langle,\rangle}\left(\gamma^{\star}\right) \neq 0$.

Thus $\left(T^{4} G, J_{4}, g_{4}\right)$ is balanced, $\left(T^{5} G, J_{5}, g_{5}\right)$ is Calabi-Yau with torsion and, for any $k \neq 4,\left(T^{k} G, J_{k}, g_{k}\right)$ is locally conformally balanced not balanced.

Let us compute the Koszul forms of an affine-Riemann manifold in local affine coordinates.

Proposition 6.1. Let $(M, \nabla,\langle\rangle$,$) be an affine-Riemann manifold. For any system of affine coordinates$ $\left(x_{1}, \ldots, x_{n}\right)$,

$$
\alpha=\frac{1}{2} d \ln (\operatorname{det} G) \quad \text { and } \quad \xi=\sum_{j=1}^{n}\left(\sum_{h, k} \mu^{k h} \frac{\partial \mu_{j h}}{\partial x_{k}}\right) d x_{j}-\alpha,
$$

where $\mu_{h k}=\left\langle\partial_{x_{h}}, \partial_{x_{k}}\right\rangle$ and the matrix $\left(\mu^{h k}\right)_{1 \leq h, k \leq n}=G^{-1}$ where $G=\left(\mu_{h k}\right)_{1 \leq h, k \leq n}$.

Proof. Let $\left(x_{1}, \ldots, x_{n}\right)$ be a system of affine coordinates. The Riemannian volume $\mu$ is given by

$$
\mu=\sqrt{\operatorname{det} G} d x_{1} \wedge \ldots \wedge d x_{n}
$$

and the first formula is a consequence of the relation $\nabla_{X} \mu=\alpha(X) \mu$ for any $X \in \Gamma(T M)$ (see Proposition 3.1).

On the other hand, we consider a local orthonormal frame $\left(E_{1}, \ldots, E_{n}\right)$ of $\langle$,$\rangle and we denote by P=$ $\left(p_{i j}\right)_{1 \leq i, j \leq n}$ the passage matrix from $\left(\partial_{x_{1}}, \ldots, \partial_{x_{n}}\right)$ to $\left(E_{1}, \ldots, E_{n}\right)$. We have $P^{t} G P=\mathrm{I}_{\mathrm{n}}$. For any $j=1, \ldots, n$,

$$
\begin{aligned}
\alpha\left(\partial_{x_{j}}\right) & =\sum_{i=1}^{n}\left\langle\gamma_{\partial_{j}} E_{i}, E_{i}\right\rangle \\
& =\sum_{i, h, k} p_{k i} p_{h i}\left\langle D_{\partial_{x_{j}}} \partial_{x_{k}}, \partial_{x_{h}}\right\rangle \\
& =\frac{1}{2} \sum_{h, k} m_{k h}\left(\frac{\partial \mu_{h k}}{\partial x_{j}}+\frac{\partial \mu_{j h}}{\partial x_{k}}-\frac{\partial \mu_{j k}}{\partial x_{h}}\right) .
\end{aligned}
$$

In the same way,

$$
\begin{aligned}
\xi\left(\partial_{x_{j}}\right) & =\sum_{i=1}^{n}\left\langle\gamma_{E_{i}} E_{i}, \partial_{x_{j}}\right\rangle \\
& =\sum_{i, h, k} p_{k i} p_{h i}\left\langle D_{\partial_{x_{h}}} \partial_{x_{k}}, \partial_{x_{j}}\right\rangle \\
& =\frac{1}{2} \sum_{h, k} m_{k h}\left(\frac{\partial \mu_{k j}}{\partial x_{h}}+\frac{\partial \mu_{j h}}{\partial x_{k}}-\frac{\partial \mu_{h k}}{\partial x_{j}}\right) \\
& =\sum_{h, k} m_{k h} \frac{\partial \mu_{j h}}{\partial x_{k}}-\alpha\left(\partial_{x_{j}}\right),
\end{aligned}
$$

where $m_{k h}=\sum_{i=1}^{n} p_{k i} p_{h i}$. But $\left(m_{k h}\right)_{1 \leq k, h \leq n}=P P^{t}$ and the result follows from the formula $P^{t} G P=\mathrm{I}_{n}$.

Example 2. Let $f: \mathbb{R}^{2} \longrightarrow \mathbb{R}$ be a smooth function. Consider the affine-Riemann manifold $\left(\mathbb{R}^{2}, \nabla^{0},\langle\rangle,\right)$ where $\nabla^{0}$ is the canonical connection of $\mathbb{R}^{2}$ and

$$
\langle,\rangle=\left(\begin{array}{cc}
\cosh (f(x, y)) & \sinh (f(x, y)) \\
\sinh (f(x, y)) & \cosh (f(x, y))
\end{array}\right) .
$$

Then $\operatorname{det}\langle\rangle=$,1 and, by virtue of Proposition 6.1, $\alpha=0$. According to Proposition 5.3, the Chern Ricci form of $\left(T \mathbb{R}^{2}, J_{1}, g_{1}\right)$ vanishes. 
The following theorem gives a large class of balanced metrics non-Kähler on $\mathbb{C}^{2}$ endowed with its canonical complex structure.

Theorem 6.2. We consider $M=\mathbb{R}^{2}$ endowed with its canonical affine structure and $\langle\rangle=,\left(\begin{array}{ll}\mu_{11} & \mu_{12} \\ \mu_{12} & \mu_{22}\end{array}\right)$ a Riemannian metric. Then $\left(T M, J_{1}, g_{1}\right)$ is balanced if and only if there exist smooth functions $v: \mathbb{R}^{2} \longrightarrow \mathbb{R}$, $f, h: \mathbb{R} \longrightarrow \mathbb{R}$ such that

$$
\mu_{12}=v, \mu_{11}\left(x_{1}, x_{2}\right)=f\left(x_{1}\right)+\int \frac{\partial v}{\partial x_{1}}\left(x_{1}, x_{2}\right) d x_{2} \quad \text { and } \quad \mu_{22}\left(x_{1}, x_{2}\right)=h\left(x_{2}\right)+\int \frac{\partial v}{\partial x_{2}}\left(x_{1}, x_{2}\right) d x_{1} .
$$

Proof. According to Proposition 6.1,

$$
\begin{aligned}
(\xi-\alpha)\left(\partial_{x_{1}}\right) & =\mu^{11} \frac{\partial \mu_{11}}{\partial x_{1}}+\mu^{12}\left(\frac{\partial \mu_{11}}{\partial x_{2}}+\frac{\partial \mu_{12}}{\partial x_{1}}\right)+\mu^{22} \frac{\partial \mu_{12}}{\partial x_{2}}-\frac{1}{\operatorname{det} G} \frac{\partial\left(\mu_{11} \mu_{22}-\mu_{12}^{2}\right)}{\partial x_{1}} \\
& =\frac{1}{\operatorname{det} G}\left(\mu_{22} \frac{\partial \mu_{11}}{\partial x_{1}}-\mu_{12}\left(\frac{\partial \mu_{11}}{\partial x_{2}}+\frac{\partial \mu_{12}}{\partial x_{1}}\right)+\mu_{11} \frac{\partial \mu_{12}}{\partial x_{2}}\right)-\frac{1}{\operatorname{det} G} \frac{\partial\left(\mu_{11} \mu_{22}-\mu_{12}^{2}\right)}{\partial x_{1}} \\
& =\frac{1}{\operatorname{det} G}\left(-\mu_{12}\left(\frac{\partial \mu_{11}}{\partial x_{2}}-\frac{\partial \mu_{12}}{\partial x_{1}}\right)+\mu_{11}\left(\frac{\partial \mu_{12}}{\partial x_{2}}-\frac{\partial \mu_{22}}{\partial x_{1}}\right)\right), \\
(\xi-\alpha)\left(\partial_{x_{2}}\right) & =\mu^{11} \frac{\partial \mu_{12}}{\partial x_{1}}+\mu^{12}\left(\frac{\partial \mu_{12}}{\partial x_{2}}+\frac{\partial \mu_{22}}{\partial x_{1}}\right)+\mu^{22} \frac{\partial \mu_{22}}{\partial x_{2}}-\frac{1}{\operatorname{det} G} \frac{\partial\left(\mu_{11} \mu_{22}-\mu_{12}^{2}\right)}{\partial x_{2}} \\
& =\frac{1}{\operatorname{det} G}\left(\mu_{22} \frac{\partial \mu_{12}}{\partial x_{1}}-\mu_{12}\left(\frac{\partial \mu_{12}}{\partial x_{2}}+\frac{\partial \mu_{22}}{\partial x_{1}}\right)+\mu_{11} \frac{\partial \mu_{22}}{\partial x_{2}}\right)-\frac{1}{\operatorname{det} G} \frac{\partial\left(\mu_{11} \mu_{22}-\mu_{12}^{2}\right)}{\partial x_{2}} \\
& =\frac{1}{\operatorname{det} G}\left(-\mu_{22}\left(\frac{\partial \mu_{11}}{\partial x_{2}}-\frac{\partial \mu_{12}}{\partial x_{1}}\right)+\mu_{12}\left(\frac{\partial \mu_{12}}{\partial x_{2}}-\frac{\partial \mu_{22}}{\partial x_{1}}\right)\right) .
\end{aligned}
$$

Thus, the vanishing of $\xi-\alpha$ is equivalent to

$$
\frac{\partial \mu_{11}}{\partial x_{2}}-\frac{\partial \mu_{12}}{\partial x_{1}}=\frac{\partial \mu_{12}}{\partial x_{2}}-\frac{\partial \mu_{22}}{\partial x_{1}}=0
$$

and we get the desired result.

Example 3. For any smooth functions $f, h: \mathbb{R} \longrightarrow \mathbb{R}$, the metric

$$
\langle,\rangle=\left(\begin{array}{cc}
e^{x+y}+e^{f(x)} & e^{x+y} \\
e^{x+y} & e^{x+y}+e^{h(y)}
\end{array}\right)
$$

satisfies the condition of the last corollary and hence defines a balanced Hermitian metric on $\mathbb{C}^{2}$.

The following theorem gives a large class of balanced metrics non-Kähler and also Calabi-Yau with torsion metrics on $\mathbb{C}^{m}$ endowed with its canonical complex structure.

Theorem 6.3. We consider $M=\mathbb{R}^{n}$ endowed its canonical affine structure and $\langle\rangle=,\operatorname{Diag}\left(\mu_{1}, \ldots, \mu_{n}\right) a$ Riemannian metric. For $k_{0} \geq 1,\left(T^{k_{0}} M, J_{k_{0}}, g_{k_{0}}\right)$ is balanced if and only if there exists $\left(f_{1}, \ldots, f_{n}\right)$ a family of positive functions such that, for $j=1, \ldots, n$,

$$
\frac{\partial f_{j}}{\partial x_{j}}=0 \quad \text { and } \quad \mu_{j}=\frac{f_{1} \ldots f_{n}}{f_{j}^{\left(n 2^{k_{0}-1}-1\right)}} .
$$

In this case, $\left(T^{k_{0}+1} M, J_{k_{0}+1}, g_{k_{0}+1}\right)$ is Calabi-Yau with torsion and, for any $k \neq k_{0},\left(T^{k} M, J_{k}, g_{k}\right)$ is locally conformally balanced.

Proof. Note first that, by virtue of (20), for any $k \geq 1$,

$$
\xi-\left(2^{k}-1\right) \alpha=-\sum_{j=1}^{n} \frac{\partial\left(\ln \left(\rho_{j}\right)\right)}{\partial x_{j}} d x_{j} \quad \text { and } \quad \rho_{j}=\frac{\left(\mu_{1} \ldots \mu_{n}\right)^{2^{k-1}}}{\mu_{j}},
$$


and hence, according to Theorem 6.1, $\left(T^{k_{0}} M, J_{k_{0}}, g_{k_{0}}\right)$ is balanced if and only if, for $j=1, \ldots, n$,

$$
\frac{\partial \rho_{j}}{\partial x_{j}}=0 \quad \text { and } \quad \rho_{j}=\frac{\left(\mu_{1} \ldots \mu_{n}\right)^{2^{k_{0}-1}}}{\mu_{j}} .
$$

We have obviously that $\rho_{1} \ldots \rho_{n}=\left(\mu_{1} \ldots \mu_{n}\right)^{n 2^{k_{0}-1}-1}$ and hence

$$
\mu_{j}=\frac{\left(\rho_{1} \ldots \rho_{n}\right)^{\frac{1}{n^{2} k_{0}^{-1}-1}}}{\rho_{j}} .
$$

If we put $f_{j}=\left(\rho_{j}\right) \frac{1}{n^{2^{k} 0^{-1}-1}}$, we get the desired result and Theorem 6.1 permits to conclude.

Now, we give the conditions in local coordinates so that $\left(T M, J_{1}, g_{1}\right)$ is pluriclosed.

Theorem 6.4. Let $(M, \nabla,\langle\rangle$,$) be an affine-Riemann manifold. Then \left(T M, J_{1}, g_{1}\right)$ is pluriclosed if and only if, for any affine coordinates $\left(x_{1}, \ldots, x_{n}\right)$,

$$
\frac{\partial^{2} \mu_{i k}}{\partial x_{j} \partial x_{h}}+\frac{\partial^{2} \mu_{j h}}{\partial x_{i} \partial x_{k}}=\frac{\partial^{2} \mu_{j k}}{\partial x_{i} \partial x_{h}}+\frac{\partial^{2} \mu_{i h}}{\partial x_{j} \partial x_{k}},
$$

for any $1 \leq i<j \leq n$ and $1 \leq k<h \leq n$ and where $\mu_{i j}=\left\langle\partial_{x_{i}}, \partial_{x_{j}}\right\rangle$. When $\operatorname{dim} M=2$, (21) reduces to

$$
\frac{\partial^{2} \mu_{11}}{\partial x_{2}^{2}}+\frac{\partial^{2} \mu_{22}}{\partial x_{1}^{2}}=2 \frac{\partial^{2} \mu_{12}}{\partial x_{1} \partial x_{2}}
$$

Proof. According to Proposition 4.1, $\left(T M, J_{1}, g_{1}\right)$ is pluriclosed if and only if, for any $1 \leq i<j \leq n$ and any $1 \leq k<h \leq n$,

$$
\begin{aligned}
0 & =\left\langle K\left(\partial_{x_{i}}, \partial_{x_{j}}\right) \partial_{x_{k}}, \partial_{x_{h}}\right\rangle-\left\langle\gamma_{\partial_{x_{j}}} \partial_{x_{k}}, \gamma_{\partial_{x_{i}}} \partial_{x_{h}}\right\rangle+\left\langle\gamma_{\partial_{x_{i}}} \partial_{x_{k}}, \gamma_{\partial_{x_{j}}} \partial_{x_{h}}\right\rangle \\
& =-\left\langle D_{\partial_{x_{i}}} D_{\partial_{x_{j}}} \partial_{x_{k}}, \partial_{x_{h}}\right\rangle+\left\langle D_{\partial_{x_{j}}} D_{\partial_{x_{i}}} \partial_{x_{k}}, \partial_{x_{h}}\right\rangle-\left\langle D_{\partial_{x_{j}}} \partial_{x_{k}}, D_{\partial_{x_{i}}} \partial_{x_{h}}\right\rangle+\left\langle D_{\partial_{x_{i}}} \partial_{x_{k}}, D_{\partial_{x_{j}}} \partial_{x_{h}}\right\rangle \\
& =-\partial_{x_{i}} \cdot\left\langle D_{\partial_{x_{j}}} \partial_{x_{k}}, \partial_{x_{h}}\right\rangle+\partial_{x_{j}} \cdot\left\langle D_{\partial_{x_{i}}} \partial_{x_{k}}, \partial_{x_{h}}\right\rangle \\
& =-\frac{1}{2}\left(\frac{\partial^{2} \mu_{k h}}{\partial x_{i} \partial x_{j}}+\frac{\partial^{2} \mu_{j h}}{\partial x_{i} \partial x_{k}}-\frac{\partial^{2} \mu_{k j}}{\partial x_{i} \partial x_{h}}-\frac{\partial^{2} \mu_{k h}}{\partial x_{i} \partial x_{j}}-\frac{\partial^{2} \mu_{i h}}{\partial x_{j} \partial x_{k}}+\frac{\partial^{2} \mu_{k i}}{\partial x_{j} \partial x_{h}}\right) \\
& =-\frac{1}{2}\left(\frac{\partial^{2} \mu_{j h}}{\partial x_{i} \partial x_{k}}-\frac{\partial^{2} \mu_{k j}}{\partial x_{i} \partial x_{h}}-\frac{\partial^{2} \mu_{i h}}{\partial x_{j} \partial x_{k}}+\frac{\partial^{2} \mu_{k i}}{\partial x_{j} \partial x_{h}}\right) .
\end{aligned}
$$

Corollary 6.1. We consider $M=\mathbb{R}^{n}$ endowed with its canonical affine structure and $\langle\rangle=,\operatorname{Diag}\left(\mu_{1}, \ldots, \mu_{n}\right)$. Then $\left(T M, J_{1}, g_{1}\right)$ is pluriclosed if and only if, for any $i \neq j, h \neq j$ and $h \neq i$,

$$
\frac{\partial^{2} \mu_{i}}{\partial x_{j}^{2}}+\frac{\partial^{2} \mu_{j}}{\partial x_{i}^{2}}=0 \quad \text { and } \quad \frac{\partial^{2} \mu_{i}}{\partial x_{j} \partial x_{h}}=0
$$

In particular, if we take $\mu_{i}=e^{f_{i}\left(x_{i}\right)}$ then $\left(T M, J_{1}, g_{1}\right)$ is pluriclosed.

Remark 2. The balanced structures constructed in Theorem 6.3 on $T M$ for $M=\mathbb{R}^{3}$ do not satisfy the equations (22) (which is in accord with the result given in [1] insuring that if a Hermitian metric is balanced and pluriclosed, then it must be Kähler).

We end this section by giving some properties of affine-Riemann manifolds with Vaisman tangent bundle.

Proposition 6.2. Let $(M, \nabla,\langle\rangle$,$) be an affine-Riemann manifold such that \left(T M, J_{1}, g_{1}\right)$ is Vaisman. Then the following assertions hold. 
1. If $\left(T M, J_{1}, g_{1}\right)$ is non-Kähler then the vector field $\Pi=\operatorname{tr}\left(\gamma^{*}\right)-\operatorname{tr}(\gamma)$ is a non-vanishing parallel vector field with respect $\nabla$ an $D$ and the distribution $\Pi^{\perp}$ is integrable and defines a codimension one totally geodesic foliation on $M$.

2. If $\operatorname{dim} M=2$ and $\left(T M, J_{1}, g_{1}\right)$ is non-Kähler then the curvature of $\langle$,$\rangle vanishes.$

Proof. According to Proposition 3.6, $\left(T M, J_{1}, g_{1}\right)$ is Vaisman if and only if (17) holds and the vector field $\Pi:=$ $\operatorname{tr}_{\langle,\rangle}\left(\gamma^{\star}-\gamma\right)$ is parallel with respect to both $D$ and $\nabla$.

1. One can deduce from (17) that if $\left(T M, J_{1}, g_{1}\right)$ is non-Kähler then $\Pi$ is non zero parallel. For any vector fields $X, Y$ orthogonal to $\Pi, D_{X} Y$ is also orthogonal to $\Pi$ and hence $F=\Pi^{\perp}$ is integrable and defines a totally geodesic foliation.

2. It is obvious that a Riemannian surface with a non zero parallel vector field is flat.

The geometry of Riemannian manifolds endowed with a codimension one totally geodesic foliation is wellunderstood (see [13]). It is then an interesting problem to study affine-Riemann manifolds with Vaisman tangent bundle.

Example 4. Consider $\mathbb{R}^{2}$ endowed with a flat Riemannian metric $\langle\rangle=,\left(\begin{array}{cc}a & b \\ b & c\end{array}\right), a, b, c \in \mathbb{R}$ and the flat torsionless connection given by

$$
\nabla_{\partial x} \partial_{x}=\partial_{y} \quad \text { and } \quad \nabla_{\partial x} \partial_{y}=\nabla_{\partial y} \partial_{y}=0
$$

Then

$$
\gamma_{\partial_{x}}^{\star}=\left(\begin{array}{cc}
\frac{c b}{a c-b^{2}} & \frac{c^{2}}{a c-b^{2}} \\
-\frac{b^{2}}{a c-b^{2}} & -\frac{c b}{a c-b^{2}}
\end{array}\right) \text { and } \gamma_{\partial_{y}}^{\star}=0 .
$$

Consider the orthonormal frame $\left(E_{1}, E_{2}\right)$ given by

$$
E_{1}=\frac{1}{\sqrt{a}} \partial_{x} \quad \text { and } \quad E_{2}=\frac{1}{\sqrt{a\left(c a-b^{2}\right)}}\left(a \partial_{y}-b \partial_{x}\right) .
$$

By using this orthonormal frame, one can check easily that

$$
\operatorname{tr}_{\langle,\rangle}\left(\gamma^{\star}\right)=0 \text { and } \operatorname{tr}_{\langle,\rangle}(\gamma)=\frac{c}{a c-b^{2}} \partial_{y} .
$$

One can also check that

$$
\gamma_{\partial_{x}}^{\star} \partial_{y}-\gamma_{\partial_{y}}^{\star} \partial_{x}=\left\langle\operatorname{tr}_{\langle,\rangle}\left(\gamma^{\star}\right)-\operatorname{tr}_{\langle,\rangle}(\gamma), \partial_{x}\right\rangle \partial_{y}-\left\langle\operatorname{tr}_{\langle,\rangle}\left(\gamma^{\star}\right)-\operatorname{tr}_{\langle,\rangle}(\gamma), \partial_{y}\right\rangle \partial_{x}
$$

and hence (17) holds. Thus $\left(T \mathbb{R}^{2}, J_{1}, g_{1}\right)$ is Vaisman and, actually this structure is left invariant.

\section{Rigid affine-Riemann manifolds}

In this section, we study the case where $(M, \nabla,\langle\rangle$,$) is an affine-Riemann manifold satisfying$

$$
D(\gamma)=0 .
$$

This condition implies that $D \alpha=D \xi=0$ and in particular $d \xi=0$. According to Theorem 6.1, we get the following result which justifies the study of this class of affine-Riemann manifolds.

Proposition 7.1. Let $(M, \nabla,\langle\rangle$,$) be an affine-Riemann manifold satisfying (23). Then, for any k \geq 1$, $\left(T^{k} M, J_{k}, g_{k}\right)$ is locally conformally balanced.

Affine-Riemann manifolds satisfying (23) will be called rigid. 
Proposition 7.2. Let $(M, \nabla,\langle\rangle$,$) be a Riemannian manifold endowed with a torsionless connection. Then the$ following assertions are equivalent:

1. $(M, \nabla,\langle\rangle$,$) is a rigid affine-Riemann manifold.$

2. The difference tensor $\gamma=D-\nabla$ satisfies:

$$
D(\gamma)=0 \quad \text { and } \quad K(X, Y)=\left[\gamma_{X}, \gamma_{y}\right]
$$

for any $X, Y \in \Gamma(T M)$.

Proof. It is a consequence of the following formula

$$
K(X, Y) Z=R^{\nabla}(X, Y) Z+D_{Y}(\gamma)(X, Z)-D_{X}(\gamma)(Y, Z)+\left[\gamma_{X}, \gamma_{Y}\right](Z),
$$

where $R^{\nabla}$ is the curvature of $\nabla$.

Le us show that we can apply the following theorem due to Kostant [19, Theorem 4] to get an interesting description of rigid affine-Riemann manifolds with complete Riemannian metric.

Theorem 7.1 (Kostant). Let $A$ be a connection on a simply-connected manifold $M$. Assume that there exists a second connection $B$ on $M$ such that

1. $B$ is invariant under parallelism, i.e., $B T=0$ and $B R=0$ where $T$ and $R$ are, respectively, the torsion and the curvature of $B$.

2. $A$ is rigid with respect to $B$, i.e., $S=B-A$ is B-parallel.

3. $M$ is complete with respect to $B$.

Let $\mathfrak{g}$ be the Lie algebra of infinitesimal $B$ affine transformations $X$ on $M$ such that $\left(L_{X}-B_{X}\right) p \in \mathfrak{s}_{p}$ for some (and hence every) point $p \in M$ where $L_{X}$ is the Lie derivative in the direction of $X$ and $\mathfrak{s}_{p}$ is the B-holonomy algebra at $p$. Then the infinitesimal action $\rho: \mathfrak{g} \longrightarrow \Gamma(T M)$ integrates to an action $\phi: G \longrightarrow \operatorname{Diff}(M)$ of a simply-connected Lie group $G$ which preserves both $A$ and $B$. Moreover, $M$ is a reductive homogeneous space with respect to the action of $G$.

Let us see that the condition (23) implies the hypothesis of Theorem 7.1 for $A=\nabla$ and $B=D$. Indeed, the condition (23) is equivalent to $\nabla$ is rigid with respect to $D$ and from (13)

$$
K(X, Y)=\left[\gamma_{X}, \gamma_{Y}\right] \text { for } X, Y \in \Gamma(T M) .
$$

Since $\gamma$ is parallel, we get that $D K=0$ and hence $D$ is invariant under parallelism. If we suppose that $M$ is simply-connected and $D$ is complete we can apply Theorem 7.1 and get the following result. Note that since $K$ is parallel the Lie algebra of holonomy is given by

$$
\mathfrak{s}_{p}=\left\{\sum K\left(u_{i}, v_{i}\right), u_{i}, v_{i} \in T_{p} M\right\} .
$$

Moreover, since $D$ is torsionless $L_{X}-B_{X}=-D X$ for any $X \in \Gamma(T M)$.

Theorem 7.2. Let $(M, \nabla,\langle\rangle$,$) be a simply-connected rigid affine-Riemann manifold such that \langle$,$\rangle is complete.$ Consider

$$
\mathfrak{g}=\left\{X \in \Gamma(T M), D X=\sum K\left(U_{i}, V_{i}\right)\right\} .
$$

Then the action $\rho: \mathfrak{g} \longrightarrow \Gamma(T M)$ integrates to an action $\phi: G \longrightarrow$ Diff $(M)$ of a simply-connected Lie group which preserves both $\nabla$ and $\langle$,$\rangle . Moreover, M$ is homogeneous reductive under this action and $(M,\langle\rangle$,$) is a$ Riemannian symmetric space.

The following result shows that there is a correspondence between simply-connected complete flat rigid affine-Riemann manifolds and associative commutative algebras. 
Corollary 7.1. Let $(M, \nabla,\langle\rangle$,$) be simply-connected rigid affine-Riemann manifold with K=0$ and $\langle$,$\rangle is$ complete. Then $(M,\langle\rangle$,$) is isometric to \mathbb{R}^{n}$ with its canonical metric and there exists an associative commutative product $\bullet$ on $\mathbb{R}^{n}$ such that $\gamma_{u} v=u \bullet v$ for any $u, v \in T_{p} \mathbb{R}^{n}$ and $p \in \mathbb{R}^{n}$.

Conversely, let $(A, \bullet)$ be a real finite dimensional associative commutative algebra and $\langle$,$\rangle a scalar product$ on $A$. The product $\bullet$ defines on $A$ a flat torsionless connection $\nabla$ and $(A, \nabla,\langle\rangle$,$) is a rigid affine-Riemann$ manifold.

Proof. Since $K=0$ and $\langle$,$\rangle is complete then (M,\langle\rangle$,$) is isometric to \mathbb{R}^{n}$ with its canonical metric. Moreover, $\gamma$ is parallel and hence it is given by $\gamma_{u} v=u \bullet v$ for any $u, v \in T_{p} \mathbb{R}^{n}$ and $p \in \mathbb{R}^{n}$, where $\bullet$ is a commutative product on $\mathbb{R}^{n}$. The relation $K(u, v)=\left[\gamma_{u}, \gamma_{v}\right]=0$ implies that $\bullet$ is associative. The converse is obvious.

Theorem 7.2 suggests us to look for rigid affine-Riemann manifolds among symmetric spaces and we give now a practical method to achieve this task. The following result is a consequence of Proposition 7.2 and the properties of the holonomy representation of symmetric spaces (see [5, Proposition 10.79]).

Proposition 7.3. Let $(M,\langle\rangle$,$) be a simply-connected Riemannian symmetric space and G$ its group of isometries, $o$ a fixed point of $M$ and $\gamma^{0}: T_{o} M \times T_{o} M \longrightarrow T_{o} M$ a symmetric product such that

$$
\operatorname{ad}(a)\left(\gamma_{u}^{0} v\right)=\gamma_{\operatorname{ad}(a) u}^{0} v+\gamma_{u}^{0} \operatorname{ad}(a) v \quad \text { and } \quad K(u, v)=\left[\gamma_{u}^{0}, \gamma_{v}^{0}\right] \quad u, v \in T_{o} M, a \in \mathfrak{g}_{0},
$$

where $\mathfrak{g}_{0}$ is the Lie algebra of the isotropy at $o$, ad $: \mathfrak{g}_{0} \longrightarrow \operatorname{End}\left(T_{o} M\right)$ is the infinitesimal isotropy representation and $K$ is the curvature of $\langle$,$\rangle . Then \gamma^{0}$ is invariant by holonomy and defines a parallel tensor $\gamma$ on M. Moreover, $(M, D-\gamma,\langle\rangle$,$) is a rigid affine-Riemann manifold.$

We illustrate this proposition by the following example.

Example 5. Consider $M:=\mathrm{SPD}(n)$ the set of real symmetric positive definite $n \times n$ matrices, which is an open subset of $\mathrm{S}(n)$ : the vector space of real symmetric $n \times n$ matrices. The connected Lie group $G:=\mathrm{GL}^{+}(n, \mathbb{R})$ of positive determinant $n \times n$ matrices acts transitively on $M: g \cdot x:=g x g^{t}$, and the isotropy subgroup at $\mathrm{I}_{n}$ is $H:=\mathrm{SO}(n)$. The Lie algebra of $H$ is $\mathfrak{h}=\mathfrak{s o}(n, \mathbb{R})$ and with $\mathfrak{m}:=\mathrm{S}(n)$, we have a canonical decomposition

$$
\mathfrak{g}=\mathfrak{h} \oplus \mathfrak{m} \quad \text { and } \quad \operatorname{Ad}(H)(\mathfrak{m}) \subset \mathfrak{m} .
$$

The scalar product on $\mathfrak{m}$ given by $\langle A, B\rangle_{0}=\operatorname{tr}(A B)$ is $\operatorname{Ad}(H)$ invariant and hence defines a G-invariant Riemannian metric $\langle$,$\rangle on M=G / H$ and $(G / H,\langle\rangle$,$) is a symmetric space. Its curvature at T_{\pi(e)} G / H=\mathfrak{m}$ is given by

$$
K(A, B) C=[[A, B], C], \quad A, B, C \in \mathfrak{m} .
$$

On the other hand, the product $\gamma^{0}: \mathfrak{m} \times \mathfrak{m} \longrightarrow \mathfrak{m}$

$$
\gamma_{A}^{0} B=A B+B A
$$

satisfies

$$
K(A, B):=\left[\gamma_{A}^{0}, \gamma_{B}^{0}\right] \quad \text { and } \quad K(A, B) \gamma_{C}^{0} E=\gamma_{K(A, B) C}^{0} E+\gamma_{C}^{0} K(A, B) E, \quad A, B, C, E \in \mathfrak{m} .
$$

Since the holonomy Lie algebra of $(G / H,\langle\rangle$,$) is generated by K$ and $G / H$ is simply-connected and by using Proposition 7.2, one can see that $\gamma^{0}$ defines an invariant parallel tensor field $\gamma$ on $G / H$ such that, if $D$ is the LeviCivita connection of $(G / H,\langle\rangle),,(G / H, D-\gamma,\langle\rangle$,$) is a rigid affine-Riemann manifold. Moreover, one can see$ that $\gamma=\gamma^{*}$ and hence $(G / H, D-\gamma,\langle\rangle$,$) is a Hessian manifold.$

We determine now complete rigid affine-Riemann manifolds of dimension 2 and 3 . We start with the following propositions.

Proposition 7.4. The manifold $\mathbb{R} \times S^{2}(r)$ carries a family depending on a non null real parameter of affine structures $\nabla^{c}$ such that $\left(\mathbb{R} \times S^{2}(r), \nabla^{c},\langle,\rangle_{0}\right)$ is a rigid affine-Riemann manifold where $S^{2}(r)$ is the 2-sphere of radius $r$ and $\langle,\rangle_{0}$ is the canonical metric of $\mathbb{R} \times S^{2}(r)$. Moreover, for $c= \pm \frac{\sqrt{2}}{r}$ we have $\operatorname{tr}_{\langle,\rangle_{0}}(\gamma)=0$ and hence $\left(T\left(\mathbb{R} \times S^{2}(r)\right), J_{1}, g_{1}\right)$ is Calabi-Yau with torsion. 
Proof. By virtue of Proposition 7.2, a rigid affine-Riemann structure on $\mathbb{R} \times S^{2}(r)$ is a symmetric tensor field $\gamma$ of type $(2,1)$ such that

$$
D(\gamma)=0 \quad \text { and } \quad K(X, Y)=\left[\gamma_{X}, \gamma_{Y}\right], \quad X, Y \in \Gamma\left(T\left(\mathbb{R} \times S^{2}(r)\right)\right) \text {, }
$$

where $D$ and $K$ are, respectively, the Levi-Civita and the curvature of $\langle,\rangle_{0}$. According to the holonomy principle (see [5, pp. 282]), this is equivalent to the following: for a fixed $p \in \mathbb{R} \times S^{2}(r)$, there exists $\gamma^{0}: T_{p}\left(\mathbb{R} \times S^{2}(r)\right) \times T_{p}\left(\mathbb{R} \times S^{2}(r)\right) \longrightarrow T_{p}\left(\mathbb{R} \times S^{2}(r)\right)$ such that, for any $u, v \in T_{p}\left(\mathbb{R} \times S^{2}(r)\right)$ and any $h \in \mathfrak{g} p$,

$$
h . \gamma_{u}^{0} v=\gamma_{h . u}^{0} v+\gamma_{u}^{0}(h . v) \quad \text { and } \quad K(u, v)=\left[\gamma_{u}^{0}, \gamma_{u}^{0}\right] \text {, }
$$

where $\mathfrak{g}_{p}$ is the holonomy Lie algebra at $p$. Take $p=(1,(0,0,1))$, denote by $\left(e_{1}, e_{2}, e_{3}\right)$ the canonical basis of $\mathbb{R}^{3}$ and $e_{0}$ the generator of $\mathbb{R}$. Then $T_{p}\left(\mathbb{R} \times S^{2}(r)\right)=\operatorname{span}\left(e_{0}, e_{1}, e_{2}\right)$ and

$$
\mathfrak{g}_{p}=\left\{\left(\begin{array}{ccc}
0 & 0 & 0 \\
0 & 0 & \lambda \\
0 & -\lambda & 0
\end{array}\right), \lambda \in \mathbb{R}\right\} .
$$

Put $\gamma_{u}^{0} v=A_{0}(u, v) e_{0}+A_{1}(u, v) e_{1}+A_{2}(u, v) e_{2}$. Then the first equation in (24) is equivalent to

$$
\left\{\begin{array}{l}
A_{0}(h . u, v)+A_{0}(u, h . v)=0, \\
A_{1}(h . u, v)+A_{1}(u, h . v)=\lambda A_{2}(u, v), \\
A_{2}(h . u, v)+A_{2}(u, h . v)=-\lambda A_{1}(u, v),
\end{array}\right.
$$

for any $u, v \in T_{p}\left(\mathbb{R} \times S^{2}(r)\right)$ and $h \in \mathfrak{g} p$. The solutions of this system of equations are given by their matrices in $\left(e_{0}, e_{1}, e_{2}\right)$

$$
A_{0}=\left(\begin{array}{ccc}
a_{1,1} & 0 & 0 \\
0 & a_{3,3} & 0 \\
0 & 0 & a_{3,3}
\end{array}\right), A_{1}=\left(\begin{array}{ccc}
0 & c_{1,3} & -c_{1,2} \\
c_{1,3} & 0 & 0 \\
-c_{1,2} & 0 & 0
\end{array}\right) \text { and } A_{2}=\left(\begin{array}{ccc}
0 & c_{1,2} & c_{1,3} \\
c_{1,2} & 0 & 0 \\
c_{1,3} & 0 & 0
\end{array}\right)
$$

and hence

$$
\gamma_{e_{0}}^{0}=\left(\begin{array}{ccc}
a_{1,1} & 0 & 0 \\
0 & c_{1,3} & -c_{1,2} \\
0 & c_{1,2} & c_{1,3}
\end{array}\right), \gamma_{e_{1}}^{0}=\left(\begin{array}{ccc}
0 & a_{3,3} & 0 \\
c_{1,3} & 0 & 0 \\
c_{1,2} & 0 & 0
\end{array}\right) \quad \text { and } \gamma_{e_{2}}^{0}=\left(\begin{array}{ccc}
0 & 0 & a_{3,3} \\
-c_{1,2} & 0 & 0 \\
c_{1,3} & 0 & 0
\end{array}\right) .
$$

The second equation of (24) is equivalent to

$$
\begin{aligned}
K\left(e_{0}, e_{1}\right) & =\left(\begin{array}{ccc}
0 & a_{1,1} a_{3,3}-c_{1,3} a_{3,3} & a_{3,3} c_{1,2} \\
-a_{1,1} c_{1,3}-c_{1,2}{ }^{2}+c_{1,3}{ }^{2} & 0 & 0 \\
-a_{1,1} c_{1,2}+2 c_{1,2} c_{1,3} & 0 & 0
\end{array}\right), \\
K\left(e_{0}, e_{2}\right) & =\left(\begin{array}{ccc}
a_{1,1} c_{1,2}-2 c_{1,2} c_{1,3} & 0 & a_{1,1} a_{3,3}-c_{1,3} a_{3,3} \\
-a_{1,1} c_{1,3}-c_{1,2}{ }^{2}+c_{1,3}^{2} & 0 & 0
\end{array}\right), \\
K\left(e_{1}, e_{2}\right) & =\left(\begin{array}{ccc}
-2 a_{3,3} c_{1,2} & 0 & 0 \\
0 & a_{3,3} c_{1,2} & c_{1,3} a_{3,3} \\
0 & -c_{1,3} a_{3,3} & a_{3,3} c_{1,2}
\end{array}\right) .
\end{aligned}
$$


But $K\left(e_{0}, e_{1}\right)=K\left(e_{0}, e_{2}\right)=0$ and $K\left(e_{1}, e_{2}\right)=\frac{1}{r^{2}}\left(E_{32}-E_{23}\right)\left(E_{i j}\right.$ is the matrix with 1 in the $i$-row and the $j$-column and zero elsewhere). This is equivalent to $c_{1,2}=0$ and $a_{1,1}=c_{1,3}=-\frac{1}{a_{3,3} r^{2}}$ and hence $\gamma^{0}$ is given by

$$
\gamma_{e_{0}}^{0}=\left(\begin{array}{ccc}
c_{1,3} & 0 & 0 \\
0 & c_{1,3} & 0 \\
0 & 0 & c_{1,3}
\end{array}\right), \gamma_{e_{1}}^{0}=\left(\begin{array}{ccc}
0 & -\frac{1}{c_{1,3} r^{2}} & 0 \\
c_{1,3} & 0 & 0 \\
0 & 0 & 0
\end{array}\right) \text { and } \gamma_{e_{2}}^{0}=\left(\begin{array}{ccc}
0 & 0 & -\frac{1}{c_{1,3} r^{2}} \\
0 & 0 & 0 \\
c_{1,3} & 0 & 0
\end{array}\right) .
$$

One can see that

$$
\operatorname{tr}_{\langle,\rangle_{0}}\left(\gamma^{0}\right)=\left(c_{1,3}-\frac{2}{c_{1,3} r^{2}}\right) e_{0} \quad \text { and } \quad \operatorname{tr}_{\langle,\rangle_{0}}\left(\left(\gamma^{0}\right)^{\star}\right)=3 c_{1,3} e_{0}
$$

Proposition 7.5. Let $(G, \nabla,\langle\rangle$,$) be a three dimensional Lie group endowed with a left invariant rigid affine-$ Riemann structure such that $\langle$,$\rangle is not flat. Then the Lie algebra of G$ is isomorphic to $\mathbb{R}^{3}$ with the non-vanishing Lie brackets

$$
\left[e_{3}, e_{1}\right]=e_{2},\left[e_{3}, e_{2}\right]=2 e_{2}
$$

and the matrices in $\left(e_{1}, e_{2}, e_{3}\right)$ of the metric and the difference tensor at e are given by

$$
\left\{\begin{array}{l}
\langle,\rangle=\left(\begin{array}{ccc}
1 & \frac{1}{2} & 0 \\
\frac{1}{2} & 1 & 0 \\
0 & 0 & v
\end{array}\right), v>0 \\
\gamma_{e_{1}}=\left(\begin{array}{ccc}
\frac{v r^{2}+1}{v r} & \frac{2}{v r} & 0 \\
\frac{1}{2} \frac{v r^{2}-1}{v r} & \frac{v r^{2}-1}{v r} & 0 \\
0 & 0 & r
\end{array}\right), \gamma_{e_{2}}=\left(\begin{array}{ccc}
\frac{2}{v r} & \frac{4}{v r} & 0 \\
\frac{v r^{2}-1}{v r} & -\frac{2}{v r} & 0 \\
0 & 0 & 0
\end{array}\right), \gamma_{e_{3}}=\left(\begin{array}{ccc}
0 & 0 & \frac{4}{r} \\
0 & 0 & -\frac{2}{r} \\
r & 0 & 0
\end{array}\right), \quad r \neq 0 .
\end{array}\right.
$$

Moreover,

$$
\operatorname{tr}_{\langle,\rangle}(\gamma)=\frac{2\left(v r^{2}+6\right)}{3 v r}\left(2 e_{1}-e_{2}\right) \quad \text { and } \operatorname{tr}_{\langle,\rangle}\left(\gamma^{*}\right)=2 r\left(2 e_{1}-e_{2}\right) \text {. }
$$

For $v=\frac{3}{\left(3\left(2^{k-1}\right)-2\right) r^{2}}$ we have $\operatorname{tr}_{\langle,\rangle}(\gamma)=\left(2^{k}-1\right) \operatorname{tr}_{\langle,\rangle}\left(\gamma^{*}\right)$ and hence $\left(T^{k} G, J_{k}, g_{k}\right)$ is balanced and $\left(T^{k+1} G, J_{k+1}, g_{k+1}\right)$ is Calabi-Yau with torsion.

Proof. If $(G, \nabla,\langle\rangle$,$) is rigid then, as a Riemannian manifold, it is symmetric and hence it is either irreducible$ and hence Einstein or it is the product of $\mathbb{R}$ with a complete Riemannian surface of constant curvature. So the Ricci curvature has signature $(+,+,+),(-,-,-),(0,+,+)$ or $(0,-,-)$. It is known that no three dimensional Lie group carries a left invariant Riemannian metric of Ricci signature $(0,+,+)$ (see [21]) and if the Ricci signature is $(+,+,+)$ then $G$ is compact simple and it is known (see [16]) that $G$ cannot carry a left invariant flat and torsionless connection. So the Ricci signature is either $(-,-,-)$ or $(0,-,-)$. According to the determination by Ha and Lee in [14, Tables 1 and 2] of the Ricci signatures of left invariant metrics on three dimensional Lie groups, the Lie algebra $\mathfrak{g}$ of $G$ and the metric are of the following forms:

1. $\mathfrak{g}_{1}=\mathbb{R}^{3}$, the non-vanishing Lie brackets are: $\left[e_{3}, e_{1}\right]=e_{1},\left[e_{3}, e_{2}\right]=e_{2}$ and $\left.\langle\rangle=,\operatorname{Diag}(1,1, v), v\right\rangle 0$,

2. $\mathfrak{g}_{2}=\mathbb{R}^{3}$, the non-vanishing Lie brackets are: $\left[e_{3}, e_{1}\right]=e_{2},\left[e_{3}, e_{2}\right]=2 e_{2}$ and $\langle\rangle=,\left(\begin{array}{ccc}1 & \frac{1}{2} & 0 \\ \frac{1}{2} & 1 & 0 \\ 0 & 0 & v\end{array}\right)$.

Now, according to Proposition 7.2, $(G, \nabla,\langle\rangle$,$) is rigid if and only if the difference tensor at e, \gamma: \mathfrak{g} \times \mathfrak{g} \longrightarrow \mathfrak{g}$ satisfies, for any $u, v \in \mathfrak{g}$,

$$
\left[\mathrm{L}_{u}, \gamma_{v}\right]=\gamma_{\mathrm{L}_{u} v} \quad \text { and } \quad K(u, v)=\left[\gamma_{u}, \gamma_{v}\right]
$$


where $\mathrm{L}_{u}$ is the left multiplication of the Levi-Civita product given by

$$
2\left\langle\mathrm{~L}_{u} v, w\right\rangle=\langle[u, v], w\rangle+\langle[w, v], u\rangle+\langle[w, u], v\rangle
$$

and $K(u, v)=\mathrm{L}_{[u, v]}-\left[\mathrm{L}_{u}, \mathrm{~L}_{v}\right]$.

A direct computation using Maple shows that when $\mathfrak{g}=\mathfrak{g}_{1}$ there is no solution and in the second case we find the $\gamma_{e_{i}}$ given in the statement of the proposition. The last statement is a consequence of Theorem 6.1.

Theorem 7.3. Let $(M, \nabla,\langle\rangle$,$) be simply-connected rigid affine-Riemann manifold with \langle$,$\rangle complete. Then:$

1. If $\operatorname{dim} M=2$ then $\langle$,$\rangle is flat.$

2. If $\operatorname{dim} M=3$ then either $\langle$,$\rangle is flat or (M, \nabla,\langle\rangle$,$) isomorphic to \mathbb{R} \times S^{2}(r)$ endowed with the rigid structure given in Proposition 7.4 or to the left invariant rigid structure given in Proposition 7.5.

Proof. Suppose that $\operatorname{dim} M=2$. Note first that the vector fields $\left(\operatorname{tr}_{\langle,\rangle}(\gamma), \operatorname{tr}_{\langle,\rangle}\left(\gamma^{\star}\right)\right)$ are $D$-parallel. $\operatorname{If} \operatorname{tr}_{\langle,\rangle}(\gamma)=$ $\operatorname{tr}_{\langle,\rangle}\left(\gamma^{\star}\right)=0$ then the curvature of $\langle$,$\rangle vanishes as we will see in Theorem 8.2. If \left(\operatorname{tr}_{\langle,\rangle}(\gamma), \operatorname{tr}_{\langle,\rangle}\left(\gamma^{\star}\right)\right) \neq(0,0)$ then there is a non zero $D$-parallel vector field on $M$ and hence the curvature of $D$ vanishes.

Suppose now that $\operatorname{dim} M=3$ and $(M,\langle\rangle$,$) is not flat. Since M$ is simply-connected and carries an affine structure it is not compact. Then $(M,\langle\rangle$,$) is a non compact simply-connected symmetric Riemannian$ manifold and hence it is the Riemannian product of a Euclidean space and a finite number of irreducible symmetric spaces (see [5, Theorem $7.76 \mathrm{pp} .194])$. Then $(M,\langle\rangle$,$) is either irreducible or it is the product of \mathbb{R}$ with a complete Riemannian surface of constant curvature. If $(M,\langle\rangle$,$) is irreducible then it is Einstein with$ nonpositive scalar curvature $s$. If $s=0$ then $(M,\langle\rangle$,$) it is Ricci-flat and hence flat since any homogeneous$ Ricci-flat Riemannian manifold is flat (see [2]). If $s<0$ then, according Alekseevskii conjecture which is true in dimension $\leq 5$ (see [5, Conjecture 7.57 pp. 190]), $(M,\langle\rangle$,$) is isometric to a solvable Lie group with a left$ invariant metric. If $(M,\langle\rangle$,$) is the product of \mathbb{R}$ with a complete Riemannian surface $S$ of constant curvature then $S$ is either the 2-dimensional hyperbolic space $H^{2}$ or $S^{2}(r)$ endowed with their canonical metric. When $S=H^{2}$ then $(M,\langle\rangle$,$) is isometric to a solvable Lie group with a left invariant metric. So far, we have shown$ that if $(M,\langle\rangle$,$) is not flat then (M,\langle\rangle$,$) is isometric to a 3-dimensional solvable Lie group with a left invariant$ Riemannian metric or $\mathbb{R} \times S^{2}(r)$.

Suppose that $(M,\langle\rangle$,$) is isometric to a 3-dimensional solvable Lie group with a left invariant metric. Let$ us show that $\nabla$ is also left invariant. According to Theorem 7.2, there exists a simply-connected Lie group $G$ which act transitively on $M$ and preserves both $\nabla$ and $\langle$,$\rangle . From the determination of the isometry groups$ of 3-dimensional solvable Lie groups (see $[9,14]$ ) one can see that the dimension of the isometry group of $(M,\langle\rangle$,$) is either 3$ or 4 and hence $\operatorname{dim} G=3$ or 4 . If $\operatorname{dim} G=4$ then $G$ contains the left multiplications and hence $\nabla$ is left invariant. If $\operatorname{dim} G=3$, the orbital map ev $: G \longrightarrow M, h \mapsto h(e)$ is a covering and hence a diffeomorphism since both $G$ and $M$ are simply-connected. Moreover, $e v$ commutes with the actions of $G$ by left multiplication on $G$ and its natural action on $M$. If we pull-back the metric $\langle$,$\rangle and \nabla$ on $G$, we get that $(M, \nabla,\langle\rangle$,$) is isomorphic to a Lie group with a left invariant connection and a left invariant metric. To$ complete the proof, we apply Propositions 7.4 and 7.5.

\section{Infinitely balanced affine-Riemann manifolds}

In this section, we introduce the notion of infinitely balanced affine-Riemann manifold (see Definition 8.1). We illustrate the importance of this class of affine-Riemann manifolds and we give some of its properties.

Definition 8.1. We call an affine-Riemann manifold $(M, \nabla,\langle\rangle$,$) infinitely balanced if its difference tensor \gamma$ satisfies $\operatorname{tr}_{\langle,\rangle}(\gamma)=\operatorname{tr}_{\langle,\rangle}\left(\gamma^{*}\right)=0$. This is equivalent to the Koszul forms satisfying $\alpha=\xi=0$.

This definition finds its justification in the following result which is a consequence of Theorem 6.1 item 3 and Propositions 5.2-5.3. 
Theorem 8.1. Let $(M, \nabla,\langle\rangle$,$) be an infinitely balanced affine-Riemann manifold with \gamma \neq 0$. Then:

1. (TM, $\left.J_{1}, g_{1}\right)$ is balanced with $\rho^{B}=\rho^{C}=0$ and it is Kähler if and only if $\gamma=\gamma^{\star}$.

2. For any $k \geq 2,\left(T^{k} M, J_{k}, g_{k}\right)$ is balanced non-Kähler with $\rho^{B}=\rho^{C}=0$.

\section{Example 6.}

In Table 5, we give many examples of infinitely balanced left invariant structures on some 6-dimensional Lie groups.

Let us start by the following remark.

Proposition 8.1. Let $(M, \nabla,\langle\rangle$,$) a compact affine-Riemann manifold such that \left(T M, J_{1}, g_{1}\right)$ is Gauduchon and $\alpha=0$. Then $\xi=0$ and hence $(M, \nabla,\langle\rangle$,$) is infinitely balanced.$

Proof. It is an immediate consequence of Proposition 3.5 and the fact that $\int_{M} d^{\star}(\alpha-\xi)=0$.

The following result describes completely the situation in dimension 2.

Theorem 8.2. Let $(M, \nabla,\langle\rangle$,$) be a connected 2-dimensional infinitely balanced affine-Riemann manifold.$ Then $\langle$,$\rangle is Hessian, i.e., \gamma=\gamma^{\star}$ and its sectional curvature is nonnegative. Moreover, if $\langle$,$\rangle is complete then$ $\nabla$ is the Levi-Civita connection of $\langle$,$\rangle and M$ is either diffeomorphic to the torus $\mathbb{T}^{2}$ or $\mathbb{R}^{2}$.

Proof. Let us first show that $\langle$,$\rangle is Hessian. Choose an orthonormal frame \left(E_{1}, E_{2}\right)$. We have in the basis $\left(E_{1}, E_{2}\right)$, since $\gamma_{E_{1}} E_{2}=\gamma_{E_{2}} E_{1}$,

$$
\gamma_{E_{1}}=\left(\begin{array}{ll}
\gamma_{11}^{1} & \gamma_{12}^{1} \\
\gamma_{11}^{2} & \gamma_{12}^{2}
\end{array}\right) \quad \text { and } \quad \gamma_{E_{2}}=\left(\begin{array}{cc}
\gamma_{12}^{1} & \gamma_{22}^{1} \\
\gamma_{12}^{2} & \gamma_{22}^{2}
\end{array}\right) .
$$

The condition $\operatorname{tr}_{\langle,\rangle}\left(\gamma^{\star}\right)=\operatorname{tr}_{\langle,\rangle} \gamma=0$ is equivalent to

$$
\gamma_{11}^{1}+\gamma_{12}^{2}=\gamma_{12}^{1}+\gamma_{22}^{2}=\gamma_{11}^{1}+\gamma_{22}^{1}=\gamma_{11}^{2}+\gamma_{22}^{2}=0
$$

Thus

$$
\gamma_{E_{1}}=\left(\begin{array}{cc}
\gamma_{11}^{1} & \gamma_{11}^{2} \\
\gamma_{11}^{2} & -\gamma_{11}^{1}
\end{array}\right) \quad \text { and } \quad \gamma_{E_{2}}=\left(\begin{array}{cc}
\gamma_{11}^{2} & -\gamma_{11}^{1} \\
-\gamma_{11}^{1} & -\gamma_{11}^{2}
\end{array}\right) \text {. }
$$

This shows that $\gamma=\gamma^{\star}$ and hence $\langle$,$\rangle is Hessian. According to (14) and (15), the curvature of \langle$,$\rangle is given by$

$$
K(X, Y)=\left[\gamma_{X}, \gamma_{Y}\right]
$$

So the curvature $\kappa: M \longrightarrow \mathbb{R}$ of $\langle$,$\rangle is given by$

$$
\kappa=\left\langle K\left(E_{1}, E_{2}\right) E_{1}, E_{2}\right\rangle=\operatorname{tr}\left(\gamma_{E_{1}}^{2}\right) \geq 0 .
$$

If $M$ is compact, according to Gauss-Bonnet's theorem,

$$
\int_{M} \kappa v=2 \pi(2-2 g) \geq 0
$$

and hence $g \leq 1$. But the case $g=0$ is not possible since the 2-sphere has no affine structure and hence $g=1$, $\kappa=0$ and then $\gamma=0$. If $M$ is non compact and $\langle$,$\rangle is complete then according to a theorem of Cohen-Vossen$ [8] $M$ is diffeomorphic to $\mathbb{R}^{2}$. But a theorem of Cheng-Yau and Pogorelov (see [23, Theorem 8.6 pp. 160]) asserts that the only Hessian metric on $\mathbb{R}^{n}$ which satisfies $\operatorname{tr}_{\langle,\rangle}(\gamma)=0$ is the canonical metric.

In dimension superior to 3, we have the following theorem about infinitely balanced Hessian affine-Riemann manifolds. 
Theorem 8.3. Let $(M, \nabla,\langle\rangle$,$) be an affine-Riemann manifold satisfying \gamma=\gamma^{\star}$ and $\operatorname{tr}_{\langle,\rangle}(\gamma)=0$. Then the Ricci curvature of $\langle$,$\rangle is nonnegative and \langle$,$\rangle is Ricci-flat if and only if \gamma=0$. Moreover, if $M$ is compact then $\gamma=0$ and hence $\nabla$ is the Levi-Civita connection of $\langle$,$\rangle .$

Proof. The condition $\gamma=\gamma^{\star}$ implies by virtue of (14) and (15) that, for any $X, Y \in \Gamma(T M)$,

$$
K(X, Y)=\left[\gamma_{X}, \gamma_{Y}\right] .
$$

Since $\operatorname{tr}_{\langle,\rangle}(\gamma)=0$, we get that the Ricci curvature is given by

$$
\operatorname{ric}(X, X)=\operatorname{tr}\left(\gamma_{X}^{2}\right) \geq 0
$$

and $\operatorname{ric}(X, X)=0$ if and only if $\gamma_{X}=0$. If $M$ is compact then according to [23, Theorem $8.8 \mathrm{pp}$. 162] $\gamma=0$ and $\nabla$ is the Levi-Civita connection of $\langle$,$\rangle .$

If we drop the hypothesis $M$ compact there are non trivial infinitely balanced Hessian affine-Riemannian manifolds.

Theorem 8.4. Let $n \geq 2$ and $c>0$. On $\mathbb{R}^{n} \backslash\{0\}$ endowed with its affine connection $\nabla^{0}$ and its canonical Euclidean product $\langle,\rangle_{0}$, consider the smooth function

$$
f\left(x_{1}, \ldots, x_{n}\right)=\int_{0}^{r}\left(t^{n}+c\right)^{\frac{1}{n}} d t
$$

where $r=\sqrt{x_{1}^{2}+\ldots+x_{n}^{2}}$ and the matrix $\langle\rangle=,\left(\frac{\partial^{2} f}{\partial x_{i} \partial_{x_{j}}}\right)_{1 \leq i, j \leq n}$. Then $\langle$,$\rangle is a Riemannian metric and \left(\mathbb{R}^{n}\right\rangle$ $\left.\{0\}, \nabla^{0},\langle\rangle,\right)$ is an affine-Riemann manifold satisfying $\gamma=\gamma^{\star}$ and $\operatorname{tr}_{\langle,\rangle}(\gamma)=0$. Moreover, for any $i \neq j$ and any $u, v \in T_{X}\left(\mathbb{R}^{n} \backslash\{0\}\right)$

$$
\left\{\begin{array}{l}
\left\langle\partial_{x_{i}}, \partial_{x_{i}}\right\rangle=\frac{r^{n+2}+c\left(r^{2}-x_{i}^{2}\right)}{r^{3}\left(r^{n}+c\right)^{\frac{n-1}{n}}},\left\langle\partial_{x_{i}}, \partial_{x_{j}}\right\rangle=-\frac{c x_{i} x_{j}}{r^{3}\left(r^{n}+c\right)^{\frac{n-1}{n}}}, \\
\langle u, v\rangle=\frac{1}{r^{3}\left(r^{n}+c\right)^{\frac{n-1}{n}}}\left(\left(r^{n}+c\right) r^{2}\langle u, v\rangle_{0}-c\langle u, X\rangle_{0}\langle v, X\rangle_{0}\right), X=\left(x_{1}, \ldots, x_{n}\right)
\end{array}\right.
$$

and the Ricci curvature of $\langle$,$\rangle is nonnegative.$

Proof. The function $f$ is smooth on $\mathbb{R}^{n} \backslash\{0\}$ and it is easy to show that, for any $i \neq j$,

$$
\frac{\partial^{2} f}{\partial x_{i}^{2}}=\frac{r^{n+2}+c\left(r^{2}-x_{i}^{2}\right)}{r^{3}\left(r^{n}+c\right)^{\frac{n-1}{n}}} \text { and } \frac{\partial^{2} f}{\partial_{x_{i}} \partial_{x_{j}}}=-\frac{c x_{i} x_{j}}{r^{3}\left(r^{n}+c\right)^{\frac{n-1}{n}}} .
$$

Let $\left(e_{1}, \ldots, e_{n}\right)$ be the canonical basis of $\mathbb{R}^{n}$. Let us show that $\langle$,$\rangle is definite positive. For any X=$ $\left(x_{1}, \ldots, x_{n}\right) \in \mathbb{R}^{n} \backslash\{0\}$ and $u \in T_{X}\left(\mathbb{R}^{n} \backslash\{0\}\right)$, we have

$$
\begin{aligned}
\left(r^{3}\left(r^{n}+c\right)^{\frac{n-1}{n}}\right)\langle u, u\rangle & =\sum_{i=1}^{n}\left(r^{2}\left(r^{n}+c\right)-c x_{i}^{2}\right) u_{i}^{2}-c \sum_{i \neq j} x_{i} x_{j} u_{i} u_{j} \\
& =r^{2}\left(r^{n}+c\right) \sum_{i=1}^{n} u_{i}^{2}-c\left(x_{1} u_{1}+\ldots+x_{n} u_{n}\right)^{2} \\
& =\left(r^{n}+c\right)|X|_{0}^{2}|u|_{0}^{2}-c\langle u, X\rangle_{0}^{2} .
\end{aligned}
$$

By virtue of Schwartz inequality

$$
c\langle u, X\rangle_{0}^{2} \leq c|X|_{0}^{2}|u|_{0}^{2} \leq\left(r^{n}+c\right)|X|_{0}^{2}|u|_{0}^{2} .
$$

This shows that $\langle u, u\rangle \geq 0$ and $\langle u, u\rangle=0$ if and only if $u=0$. Then $\langle$,$\rangle is a Hessian metric.$ 
Let us show that $\operatorname{det}(\langle\rangle)=$,1 . Indeed, the rows $\left(L_{1}, \ldots, L_{n}\right)$ of $\langle$,$\rangle are given by$

$$
L_{i}=\frac{1}{r^{3}\left(r^{n}+c\right)^{\frac{n-1}{n}}}\left(r^{2}\left(r^{n}+c\right) e_{i}-c x_{i} \rho\right) \text { and } \rho=x_{1} e_{1}+\ldots+x_{n} e_{n} .
$$

So

$$
\begin{aligned}
\left(r^{3 n}\left(r^{n}+c\right)^{n-1}\right) \operatorname{det}(\langle,\rangle) & =\operatorname{det}\left(r^{2}\left(r^{n}+c\right) e_{1}-c x_{1} \rho, \ldots, r^{2}\left(r^{n}+c\right) e_{n}-c x_{n} \rho\right) \\
& =r^{2 n}\left(r^{n}+c\right)^{n}+\sum_{i=1}^{n} \operatorname{det}\left(r^{2}\left(r^{n}+c\right) e_{1}, \ldots, r^{2}\left(r^{n}+c\right) e_{i-1},-c x_{i} \rho, r^{2}\left(r^{n}+c\right) e_{i+1}, \ldots, r^{2}\left(r^{n}+c\right) e_{n}\right) \\
& =r^{2 n}\left(r^{n}+c\right)^{n}-c r^{2 n}\left(r^{n}+c\right)^{n-1} \\
& =r^{2 n}\left(r^{n}+c\right)^{n-1}\left(r^{n}+c-c\right)=r^{3 n}\left(r^{n}+c\right)^{n-1} .
\end{aligned}
$$

So $\operatorname{tr}_{\langle,\rangle}(\gamma)=0$ which completes the proof.

\section{Some examples of left invariant generalized Kähler structures on some 6-dimensional connected and simply-connected Lie groups}

In this section, we give examples of left invariant generalized Kähler structures on some 6-dimensional connected and simply-connected Lie groups by giving the complex isomorphism and the metric on the corresponding Lie algebras.

Our examples are based on the classification of 3-dimensional real Novikov algebras given in [7]. Recall that a Novikov algebra is a left symmetric algebra such that right multiplications commute.

Let $\left(\mathfrak{g}, . .,\langle,\rangle_{0}\right)$ be a Novikov algebra of dimension 3 endowed with a scalar product. The bracket $[a, b]=a . b-b . a$ induces on $\mathfrak{g}$ a Lie algebra structure. Let $G$ be the connected and simply-connected Lie group associated to $(\mathfrak{g},[]$,$) . Then the left symmetric product and \langle,\rangle_{0}$ induce on $G$ a left invariant affineRiemann structure $(\nabla,\langle\rangle$,$) . We have seen that on \Phi(\mathfrak{g})=\mathfrak{g} \times \mathfrak{g}$ there are a Lie bracket $[,]_{\Phi}$, a complex isomorphism $J$ and a scalar product $\langle,\rangle_{\Phi}$ given by (10) and (11). Moreover, according to Theorem 2.1, the Hermitian structure $\left(T G, J_{1}, g_{1}\right)$ associated to $(G, \nabla,\langle\rangle$,$) is diffeomorphic to \left(G \times \mathfrak{g}, J_{0}, g_{0}\right)$ where $G \times \mathfrak{g}$ is the simply-connected Lie group associated to $\left(\Phi(\mathfrak{g}),[,]_{\Phi}\right)$ and $\left(J_{0}, g_{0}\right)$ are the left invariant tensor field associated to $\left(J,\langle,\rangle_{\Phi}\right)$.

In Tables 1 and 2, for any 3-dimensional real Novikov algebra given in [7] and identified to $\mathbb{R}^{3}$ with its canonical basis $\left(e_{1}, e_{2}, e_{3}\right)$, we give its multiplication table and the Lie bracket $[,]_{\Phi}$ on $\Phi\left(\mathbb{R}^{3}\right)=\mathbb{R}^{3} \times \mathbb{R}^{3}$ in the basis $\left(f_{1}, \ldots, f_{6}\right)$ where $f_{i}=\left(e_{i}, 0\right)$ for $1=1,2,3$ and $f_{j}=\left(0, e_{j}\right)$ for $j=4,5,6$. These 6 -dimensional Lie algebras are labeled $N_{1}^{\mathfrak{g}_{1}}(a), N_{2}^{\mathfrak{g}_{1}}$ and so on. The metric $\langle,\rangle_{0}$ is given by its matrix in $\left(e_{1}, e_{2}, e_{3}\right)$,

$$
\langle,\rangle_{0}=\left(\begin{array}{lll}
g_{1,1} & g_{1,2} & g_{1,3} \\
g_{1,2} & g_{2,2} & g_{2,3} \\
g_{1,3} & g_{2,3} & g_{3,3}
\end{array}\right) .
$$

In Tables 3-8, when we refer to a 6-dimensional Lie algebra in Tables 1 and 2 having a generalized Kähler structure this means that $\left(J,\langle,\rangle_{\Phi}\right)$ are given in the basis $\left(f_{1}, \ldots, f_{6}\right)$ by

$$
J=\left(\begin{array}{cc}
0 & -\mathrm{I}_{3} \\
\mathrm{I}_{3} & 0
\end{array}\right) \text { and }\langle,\rangle_{\Phi}=\left(\begin{array}{cc}
\langle,\rangle_{0} & 0 \\
0 & \langle,\rangle_{0}
\end{array}\right)
$$

with the mentioned restrictions on the $\left(g_{i, j}\right)$.

The realization of the examples in Tables 3-8 was possible thank to the software Maple. 
Table 1: Three dimensional Novikov algebras and their associated phase Lie algebras.

\begin{tabular}{|c|c|}
\hline Left symmetric product on $\mathbb{R}^{3}$ & $e_{1} \bullet e_{1}=a e_{1}, e_{1} \bullet e_{2}=(1+a) e_{2}, e_{1} \cdot e_{3}=(1+a) e_{3}, e_{2} \bullet e_{1}=a e_{2}, e_{3} \bullet e_{1}=a e_{3}$. \\
\hline Lie brackets on $N_{1}^{\mathfrak{g}_{1}}(a)$ & $\begin{array}{l}{\left[f_{1}, f_{2}\right]=f_{2},\left[f_{1}, f_{3}\right]=f_{3},\left[f_{1}, f_{4}\right]=a f_{4},\left[f_{1}, f_{5}\right]=(1+a) f_{5},\left[f_{1}, f_{6}\right]=(1+a) f_{6},} \\
{\left[f_{2}, f_{4}\right]=a f_{5},\left[f_{3}, f_{4}\right]=a f_{6} .}\end{array}$ \\
\hline Left symmetric product on $\mathbb{R}^{3}$ & $e_{1} \cdot e_{1}=-e_{1}+e_{2}, e_{2} \cdot e_{1}=-e_{2}, e_{3} \bullet e_{1}=-e_{3}$. \\
\hline Lie brackets on $N_{2}^{\mathfrak{g}_{1}}$ & {$\left[f_{1}, f_{2}\right]=f_{2},\left[f_{1}, f_{3}\right]=f_{3},\left[f_{1}, f_{4}\right]=-f_{4}+f_{5},,\left[f_{2}, f_{4}\right]=-f_{5},\left[f_{3}, f_{4}\right]=-f_{6}$} \\
\hline$\overline{\text { Left symmetric product on } \mathbb{R}^{3}}$ & $e_{1} \bullet e_{1}=a e_{1}, e_{1} \bullet e_{2}=a e_{2}+e_{3}, e_{1} \bullet e_{3}=\alpha e_{2}+(1+a) e_{3}, e_{2} \bullet e_{1}=a e_{2}, e_{3} \bullet e_{1}=a e_{3}$ \\
\hline Lie brackets on $N_{2}^{\mathfrak{g}_{2}^{\alpha}}(a)$ & $\begin{array}{l}{\left[f_{1}, f_{2}\right]=f_{3},\left[f_{1}, f_{3}\right]=\alpha f_{2}+f_{3},\left[f_{1}, f_{4}\right]=a f_{4},\left[f_{1}, f_{5}\right]=a f_{5}+f_{6}} \\
{\left[f_{1}, f_{6}\right]=\alpha f_{5}+(1+a) f_{6},\left[f_{2}, f_{4}\right]=a f_{5},\left[f_{3}, f_{4}\right]=a f_{6}}\end{array}$ \\
\hline Left symmetric product on $\mathbb{R}^{3}$ & $\begin{array}{l}e_{1} \bullet e_{1}=a e_{1}+e_{2}, e_{1} \bullet e_{2}=a e_{2}+e_{3}, e_{1} \bullet e_{3}=\left(a+a^{2}\right) e_{2}+(1+a) e_{3}, \\
e_{2} \bullet e_{1}=a e_{2}, e_{3} \bullet e_{1}=a e_{3} .\end{array}$ \\
\hline Lie brackets on $N_{2}^{\mathfrak{g}_{2}^{a^{2}+a}}(a)$ & $\begin{array}{l}{\left[f_{1}, f_{2}\right]=f_{3},\left[f_{1}, f_{3}\right]=\left(a+a^{2}\right) f_{2}+f_{3},\left[f_{1}, f_{4}\right]=a f_{4}+f_{5},\left[f_{1}, f_{5}\right]=a f_{5}+f_{6},} \\
{\left[f_{1}, f_{6}\right]=\left(a+a^{2}\right) f_{5}+(1+a) f_{6},\left[f_{2}, f_{4}\right]=a f_{5},\left[f_{3}, f_{4}\right]=a f_{6} .}\end{array}$ \\
\hline Left symmetric product on $\mathbb{R}^{3}$ & $\begin{array}{l}e_{1} \bullet e_{1}=-\frac{1}{3} e_{1}, e_{1} \bullet e_{2}=\frac{8}{3} e_{2}-8 e_{3}, e_{1} \cdot e_{3}=\frac{7}{9} e_{2}-\frac{7}{3} e_{3}, \\
e_{2} \bullet e_{1}=\frac{8}{3} e_{2}-9 e_{3}, e_{3} \bullet e_{1}=e_{2}-\frac{10}{3} e_{3} .\end{array}$ \\
\hline Lie brackets on $N_{3}^{\mathfrak{g}^{-\frac{2}{9}}}$ & $\begin{array}{l}{\left[f_{1}, f_{2}\right]=f_{3},\left[f_{1}, f_{3}\right]=-\frac{2}{9} f_{2}+f_{3},\left[f_{1}, f_{4}\right]=-\frac{1}{3} f_{4},\left[f_{1}, f_{5}\right]=\frac{8}{3} f_{5}-8 f_{6},} \\
{\left[f_{1}, f_{6}\right]=\frac{7}{9} f_{5}-\frac{7}{3} f_{6},\left[f_{2}, f_{4}\right]=\frac{8}{3} f_{5}-9 f_{6},\left[f_{3}, f_{4}\right]=f_{5}-\frac{10}{3} f_{6} .}\end{array}$ \\
\hline Left symmetric product on $\mathbb{R}^{3}$ & $\begin{array}{l}e_{1} \bullet e_{1}=-\frac{1}{3} e_{1}+e_{2}, e_{1} \cdot e_{2}=\frac{8}{3} e_{2}-8 e_{3}, e_{1} \bullet e_{3}=\frac{7}{9} e_{2}-\frac{7}{3} e_{3}, \\
e_{2} \bullet e_{1}=\frac{8}{3} e_{2}-9 e_{3}, e_{3} \cdot e_{1}=e_{2}-\frac{10}{3} e_{3} .\end{array}$ \\
\hline Lie brackets on $N_{4}^{\mathfrak{g}_{2}^{-\frac{2}{9}}}$ & $\begin{array}{l}{\left[f_{1}, f_{2}\right]=f_{3},\left[f_{1}, f_{3}\right]=-\frac{2}{9} f_{2}+f_{3},\left[f_{1}, f_{4}\right]=-\frac{1}{3} f_{4}+e_{5},\left[f_{1}, f_{5}\right]=\frac{8}{3} f_{5}-8 f_{6},} \\
{\left[f_{1}, f_{6}\right]=\frac{7}{9} f_{5}-\frac{7}{3} f_{6},\left[f_{2}, f_{4}\right]=\frac{8}{3} f_{5}-9 f_{6},\left[f_{3}, f_{4}\right]=f_{5}-\frac{10}{3} f_{6} .}\end{array}$ \\
\hline Left symmetric product on $\mathbb{R}^{3}$ & $\begin{array}{l}e_{1} \bullet e_{1}=3 a e_{1}-\left(3 a^{2}+\frac{1}{3} a\right) e_{2}, e_{1} \cdot e_{2}=6 a e_{2}+(1-9 a) e_{3}, e_{1} \cdot e_{3}=\left(a-\frac{2}{9}\right) e_{2}+e_{3}, \\
e_{2} \cdot e_{1}=6 a e_{2}-9 a e_{3}, e_{2} \cdot e_{2}=-3 e_{2}+9 e_{3}, e_{2} \bullet e_{3}=-e_{2}+3 e_{3}, \\
e_{3} \bullet e_{1}=a e_{2}, e_{3} \cdot e_{2}=-e_{2}+3 e_{3}, e_{3} \cdot e_{3}=-\frac{1}{3} e_{2}+e_{3} .\end{array}$ \\
\hline Lie brackets on $N_{5}^{\mathfrak{g}_{2}^{-\frac{2}{9}}}(a)$ & $\begin{array}{l}{\left[f_{1}, f_{2}\right]=f_{3},\left[f_{1}, f_{4}\right]=3 a f_{4}-\left(3 a^{2}+\frac{1}{3} a\right) f_{5},\left[f_{1}, f_{5}\right]=6 a f_{5}+(1-9 a) f_{6},} \\
{\left[f_{1}, f_{3}\right]=-\frac{2}{9} f_{2}+f_{3},\left[f_{1}, f_{6}\right]=\left(a-\frac{2}{9}\right) f_{5}+f_{6},\left[f_{2}, f_{4}\right]=6 a f_{5}-9 a f_{6},\left[f_{2}, f_{5}\right]=-3 f_{5}+f_{6},} \\
{\left[f_{2}, f_{6}\right]=-f_{5}+3 f_{6},\left[f_{3}, f_{4}\right]=a f_{5},\left[f_{3}, f_{5}\right]=-f_{5}+3 f_{6},\left[f_{3}, f_{6}\right]=-\frac{1}{3} f_{5}+f_{6} .}\end{array}$ \\
\hline Left symmetric product on $\mathbb{R}^{3}$ & $\begin{array}{l}e_{1} \cdot e_{1}=-\frac{2}{3} e_{1}-\frac{8}{27} e_{2}+\frac{2}{3} e_{3}, e_{1} \cdot e_{2}=-\frac{4}{3} e_{2}+3 e_{3}, e_{1} \cdot e_{3}=-\frac{4}{9} e_{2}+e_{3}, \\
e_{2} \cdot e_{1}=-\frac{4}{3} e_{2}+2 e_{3}, e_{2} \cdot e_{2}=-3 e_{2}+9 e_{3}, e_{2} \cdot e_{3}=-e_{2}+3 e_{3}, \\
e_{3} \cdot e_{1}=-\frac{2}{9} e_{2}, e_{3} \cdot e_{2}=-e_{2}+3 e_{3}, e_{3} \cdot e_{3}=-\frac{1}{3} e_{2}+e_{3},\end{array}$ \\
\hline Lie brackets on $N_{6}^{\mathfrak{g}_{2}^{-\frac{2}{9}}}$ & $\begin{array}{l}{\left[f_{1}, f_{2}\right]=f_{3},\left[f_{1}, f_{3}\right]=-\frac{2}{9} f_{2}+f_{3},\left[f_{1}, f_{4}\right]=-\frac{2}{3} f_{4}-\frac{8}{27} f_{5}+\frac{2}{3} f_{6},\left[f_{1}, f_{5}\right]=-\frac{4}{3} f_{5}+3 f_{6},} \\
{\left[f_{1}, f_{6}\right]=-\frac{4}{9} f_{5}+f_{6},\left[f_{2}, f_{4}\right]=-\frac{4}{3} f_{5}+2 f_{6},\left[f_{2}, f_{5}\right]=-3 f_{5}+9 f_{6},\left[f_{2}, f_{6}\right]=-f_{5}+3 f_{6},} \\
{\left[f_{3}, f_{4}\right]=-\frac{2}{9} f_{5},\left[f_{3}, f_{5}\right]=-f_{5}+3 f_{6},\left[f_{3}, f_{6}\right]=-\frac{1}{3} f_{5}+f_{6},}\end{array}$ \\
\hline Left symmetric product on $\mathbb{R}^{3}$ & $\begin{array}{l}e_{1} \cdot e_{1}=-\frac{2}{3} e_{1}-\frac{11}{27} e_{2}+e_{3}, e_{1} \cdot e_{2}=-\frac{4}{3} e_{2}+3 e_{3}, e_{1} \cdot e_{3}=-\frac{4}{9} e_{2}+e_{3}, \\
e_{2} \cdot e_{1}=-\frac{4}{3} e_{2}+2 e_{3}, e_{2} \cdot e_{2}=-3 e_{2}+9 e_{3}, e_{2} \cdot e_{3}=-e_{2}+3 e_{3}, \\
e_{3} \cdot e_{1}=-\frac{2}{9} e_{2}, e_{3} \bullet e_{2}=-e_{2}+3 e_{3}, e_{3} \cdot e_{3}=-\frac{1}{3} e_{2}+e_{3},\end{array}$ \\
\hline Lie brackets on $N_{7}^{\mathfrak{g}_{2}^{-\frac{2}{9}}}$ & $\begin{array}{l}{\left[f_{1}, f_{2}\right]=f_{3},\left[f_{1}, f_{3}\right]=-\frac{2}{9} f_{2}+f_{3},\left[f_{1}, f_{4}\right]=-\frac{2}{3} f_{4}-\frac{11}{27} f_{5}+f_{6},\left[f_{1}, f_{5}\right]=-\frac{4}{3} f_{5}+3 f_{6},} \\
{\left[f_{1}, f_{6}\right]=-\frac{4}{9} f_{5}+f_{6},\left[f_{2}, f_{4}\right]=-\frac{4}{3} f_{5}+2 f_{6},\left[f_{2}, f_{5}\right]=-3 f_{5}+9 f_{6},\left[f_{2}, f_{6}\right]=-f_{5}+3 f_{6},} \\
{\left[f_{3}, f_{4}\right]=-\frac{2}{9} f_{5},\left[f_{3}, f_{5}\right]=-f_{5}+3 f_{6},\left[f_{3}, f_{6}\right]=-\frac{1}{3} f_{5}+f_{6},}\end{array}$ \\
\hline
\end{tabular}


Table 2: Three dimensional Novikov algebras and their associated phase Lie algebras (Continued).

\begin{tabular}{|c|c|}
\hline Left symmetric product on $\mathbb{R}^{3}$ & $e_{1} \bullet e_{1}=a e_{1}, e_{1} \cdot e_{2}=(1+a) e_{3}, e_{1} \cdot e_{3}=(1+a) e_{3}, e_{2} \cdot e_{1}=a e_{3}, e_{3} \cdot e_{1}=a e_{3}$ \\
\hline Lie brackets on $N_{8}^{\mathfrak{g}_{2}^{0}}(a)$ & $\begin{array}{l}{\left[f_{1}, f_{2}\right]=f_{3},\left[f_{1}, f_{3}\right]=f_{3},\left[f_{1}, f_{4}\right]=a f_{4},\left[f_{1}, f_{5}\right]=(1+a) f_{6},\left[f_{1}, f_{6}\right]=(1+a) f_{6}} \\
{\left[f_{2}, f_{4}\right]=a f_{6},\left[f_{3}, f_{4}\right]=a f_{6} .}\end{array}$ \\
\hline Left symmetric product on $\mathbb{R}^{3}$ & $e_{1} \cdot e_{1}=-e_{1}+e_{3}, e_{2} \cdot e_{1}=-e_{3}, e_{3} \cdot e_{1}=-e_{3}$ \\
\hline Lie brackets on $N_{9}^{\mathfrak{g}_{2}^{0}}$ & {$\left[f_{1}, f_{2}\right]=f_{3},\left[f_{1}, f_{3}\right]=f_{3},\left[f_{1}, f_{4}\right]=-f_{4}+f_{6},\left[f_{2}, f_{4}\right]=-f_{6},\left[f_{3}, f_{4}\right]=-f_{6}$} \\
\hline Left symmetric product on $\mathbb{R}^{3}$ & $\begin{array}{l}e_{1} \cdot e_{1}=a e_{1}, e_{1} \cdot e_{2}=a e_{2}+e_{3}, e_{1} \cdot e_{3}=(1+a) e_{3} \\
e_{2} \cdot e_{1}=a e_{2}, e_{2} \cdot e_{2}=-e_{2}+e_{3}, e_{3} \cdot e_{1}=a e_{3}\end{array}$ \\
\hline Lie brackets on $N_{10}^{\mathfrak{g}_{2}^{0}}(a)$ & $\begin{array}{l}{\left[f_{1}, f_{2}\right]=f_{3},\left[f_{1}, f_{3}\right]=f_{3},\left[f_{1}, f_{4}\right]=a f_{4},\left[f_{1}, f_{5}\right]=a f_{5}+f_{6}} \\
{\left[f_{1}, f_{6}\right]=(1+a) f_{6},\left[f_{2}, f_{4}\right]=a f_{5},\left[f_{2}, f_{5}\right]=-f_{5}+f_{6},\left[f_{3}, f_{4}\right]=a f_{6}}\end{array}$ \\
\hline Left symmetric product on $\mathbb{R}^{3}$ & $e_{1} \bullet e_{1}=-e_{1}+e_{3}, e_{1} \bullet e_{2}=-e_{2}+e_{3}, e_{2} \bullet e_{1}=-e_{2}, e_{2} \bullet e_{2}=-e_{2}+e_{3}, e_{3} \bullet e_{1}=-e_{3}$ \\
\hline Lie brackets on $N_{11}^{\mathfrak{g}_{2}^{0}}$ & $\begin{array}{l}{\left[f_{1}, f_{2}\right]=f_{3},\left[f_{1}, f_{3}\right]=f_{3},\left[f_{1}, f_{4}\right]=-f_{4}+f_{5},\left[f_{1}, f_{5}\right]=-f_{5}+f_{6}} \\
{\left[f_{2}, f_{4}\right]=-f_{5},\left[f_{2}, f_{5}\right]=-f_{5}+f_{6},\left[f_{3}, f_{4}\right]=-f_{6} .}\end{array}$ \\
\hline Left symmetric product on $\mathbb{R}^{3}$ & $e_{1} \cdot e_{1}=e_{2}, e_{1} \cdot e_{2}=(1+a) e_{3}, e_{2} \cdot e_{1}=a e_{3}$ \\
\hline Lie brackets on $N_{1}^{\mathfrak{g}_{3}}(a)$ & {$\left[f_{1}, f_{2}\right]=f_{3},\left[f_{1}, f_{4}\right]=f_{5},\left[f_{1}, f_{5}\right]=(1+a) f_{6},\left[f_{2}, f_{4}\right]=a f_{6}$} \\
\hline Left symmetric product on $\mathbb{R}^{3}$ & $e_{1} \cdot e_{1}=a e_{3}, e_{1} \cdot e_{2}=e_{3}, e_{2} \cdot e_{2}=e_{3}$ \\
\hline Lie brackets on $N_{2}^{\mathfrak{g}_{3}}(a)$ & {$\left[f_{1}, f_{2}\right]=f_{3},\left[f_{1}, f_{4}\right]=a f_{6},\left[f_{1}, f_{5}\right]=f_{6},\left[f_{2}, f_{5}\right]=f_{6}$} \\
\hline Left symmetric product on $\mathbb{R}^{3}$ & $e_{1} \bullet e_{1}=e_{3}, e_{1} \cdot e_{2}=e_{1}, e_{2} \bullet e_{1}=e_{1}-e_{3}, e_{2} \cdot e_{2}=e_{2}, e_{2} \bullet e_{3}=e_{3}, e_{3} \bullet e_{2}=e_{3}$ \\
\hline Lie brackets on $N_{3}^{\mathfrak{g}_{3}}$ & $\begin{array}{l}{\left[f_{1}, f_{2}\right]=f_{3},\left[f_{1}, f_{4}\right]=f_{6},\left[f_{1}, f_{5}\right]=f_{4},\left[f_{2}, f_{4}\right]=f_{4}-f_{6},\left[f_{2}, f_{5}\right]=f_{5},} \\
{\left[f_{2}, f_{6}\right]=f_{6},\left[f_{3}, f_{5}\right]=f_{6} .}\end{array}$ \\
\hline Left symmetric product on $\mathbb{R}^{3}$ & $e_{1} \bullet e_{2}=e_{1}, e_{2} \bullet e_{1}=e_{1}-e_{3}, e_{2} \bullet e_{2}=e_{2}, e_{2} \bullet e_{3}=e_{3}, e_{3} \bullet e_{2}=e_{3}$ \\
\hline Lie brackets on $N_{4}^{\mathfrak{g}_{3}}$ & $\begin{array}{l}{\left[f_{1}, f_{2}\right]=f_{3},\left[f_{1}, f_{5}\right]=f_{4},\left[f_{2}, f_{4}\right]=f_{4}-f_{6},\left[f_{2}, f_{5}\right]=f_{5}} \\
{\left[f_{2}, f_{6}\right]=f_{6},\left[f_{3}, f_{5}\right]=f_{6} .}\end{array}$ \\
\hline Left symmetric product on $\mathbb{R}^{3}$ & $e_{1} \cdot e_{2}=\frac{1}{2} e_{3}, e_{2} \cdot e_{1}=-\frac{1}{2} e_{3}$ \\
\hline Lie brackets on $N_{5}^{\mathfrak{g}_{3}}$ & {$\left[f_{1}, f_{2}\right]=f_{3},\left[f_{1}, f_{5}\right]=\frac{1}{2} f_{6},\left[f_{2}, f_{4}\right]=-\frac{1}{2} f_{6}$} \\
\hline Left symmetric product on $\mathbb{R}^{3}$ & $e_{1} \bullet e_{1}=a e_{1}, e_{1} \bullet e_{2}=a e_{2}+e_{3}, e_{1} \cdot e_{3}=e_{2}+a e_{3}, e_{2} \cdot e_{1}=a e_{2}, e_{3} \cdot e_{1}=a e_{3}$ \\
\hline Lie brackets on $N_{1}^{\mathfrak{g}_{4}}(a)$ & $\begin{array}{l}{\left[f_{1}, f_{2}\right]=f_{3},\left[f_{1}, f_{3}\right]=f_{2},\left[f_{1}, f_{4}\right]=a f_{4},\left[f_{1}, f_{5}\right]=a f_{5}+f_{6}} \\
{\left[f_{1}, f_{6}\right]=f_{5}+a f_{6},\left[f_{2}, f_{4}\right]=a f_{5},\left[f_{3}, f_{4}\right]=a f_{6} .}\end{array}$ \\
\hline Left symmetric product on $\mathbb{R}^{3}$ & $e_{1} \bullet e_{1}=e_{1}+e_{3}, e_{1} \bullet e_{2}=e_{2}+e_{3}, e_{1} \bullet e_{3}=e_{2}+e_{3}, e_{2} \bullet e_{1}=e_{2}, e_{3} \bullet e_{1}=e_{3}$ \\
\hline Lie brackets on $N_{2}^{\mathfrak{g}_{4}}$ & $\begin{array}{l}{\left[f_{1}, f_{2}\right]=f_{3},\left[f_{1}, f_{3}\right]=f_{2},\left[f_{1}, f_{4}\right]=f_{4}+f_{6},\left[f_{1}, f_{5}\right]=f_{5}+f_{6}} \\
{\left[f_{1}, f_{6}\right]=f_{5}+f_{6},\left[f_{2}, f_{4}\right]=f_{5},\left[f_{3}, f_{4}\right]=f_{6} .}\end{array}$ \\
\hline Left symmetric product on $\mathbb{R}^{3}$ & $e_{1} \cdot e_{1}=a e_{1}, e_{1} \cdot e_{2}=a e_{2}+e_{3}, e_{1} \cdot e_{3}=-e_{2}+a e_{3}, e_{2} \cdot e_{1}=a e_{2}, e_{3} \cdot e_{1}=a e_{3}$ \\
\hline Lie brackets on $N_{1}^{\mathfrak{g}_{5}}(a)$ & $\begin{array}{l}{\left[f_{1}, f_{2}\right]=f_{3},\left[f_{1}, f_{3}\right]=-f_{2},\left[f_{1}, f_{4}\right]=a f_{4},\left[f_{1}, f_{5}\right]=a f_{5}+f_{6}} \\
{\left[f_{1}, f_{6}\right]=-f_{5}+a f_{6},\left[f_{2}, f_{4}\right]=a f_{5},\left[f_{3}, f_{4}\right]=a f_{6} .}\end{array}$ \\
\hline
\end{tabular}


Table 3: Examples of Kähler Lie algebras.

\begin{tabular}{|c|c|}
\hline The Lie algebra & Conditions on the Hermitian metric \\
\hline$N_{1}^{\mathfrak{g}_{1}}(a)$ & {$\left[a=-2, g_{1,2}=g_{1,3}=0,0<g_{1,1}, 0<g_{3,3}, \frac{g_{2,3}{ }^{2}}{g_{3,3}}<g_{2,2}\right]$} \\
\hline$N_{2}^{\mathfrak{g}_{2}^{\alpha}}(a)$ & $\begin{array}{l}{\left[a=-2, \alpha=0, g_{1,3}=0, g_{2,3}=g_{2,2}, 0<g_{2,2}, g_{2,2}<g_{3,3},-\frac{g_{1,2}{ }^{2} g_{3,3}}{g_{2,2}\left(-g_{3,3}+g_{2,2}\right.}<g_{1,1}\right]} \\
{\left[a=-1, \alpha<0, g_{1,2}=g_{1,3}=0, g_{2,2}=-\frac{g_{3,3}}{\alpha}, g_{2,3}=\frac{g_{2,2}}{2}, 0<g_{1,1}, 0<g_{2,2}, \alpha<-\frac{1}{4}\right]}\end{array}$ \\
\hline$N_{8}^{\mathfrak{g}_{2}^{0}}(a)$ & {$\left[a=-2, g_{1,2}=0, g_{1,3}=0, g_{2,3}=g_{3,3}, 0<g_{1,1}, 0<g_{2,3}, g_{2,3}<g_{2,2}\right]$} \\
\hline$N_{10}^{\mathfrak{g}_{2}^{0}}(a)$ & {$\left[a=-2, g_{1,2}=2 g_{2,2}-2 g_{2,3}, g_{1,3}=0, g_{3,3}=g_{2,3}, 0<g_{2,3}, g_{2,3}<g_{2,2}, 4 g_{2,2}-4 g_{2,3}<g_{1,1}\right]$} \\
\hline$N_{1}^{\mathfrak{g}_{5}}(a)$ & {$\left[a=0, g_{1,2}=0, g_{1,3}=0, g_{2,2}=g_{3,3}, g_{2,3}=0,0<g_{1,1}, 0<g_{2,2}\right]$} \\
\hline
\end{tabular}

Table 4: Examples of Gauduchon Lie algebras.

\begin{tabular}{l|l}
\hline The Lie algebra & Conditions on the Hermitian metric \\
\hline$N_{1}^{\mathfrak{g}_{1}}(a)$ & {$\left[a=-2,\left(g_{1,2}, g_{1,3}\right) \neq(0,0)\right]$ or $\left[a=-\frac{4}{3}\right]$} \\
\hline$N_{2}^{\mathfrak{g}_{2}^{\alpha}}(a)$ & {$[a=-1]$ or $\left[a=-\frac{2}{3}\right]$} \\
\hline$N_{5}^{\mathfrak{g}_{2}^{-\frac{2}{9}}}(a)$ & {$\left[a=-\frac{1}{3}\right]$ or $\left[a=-\frac{2}{9}\right]$} \\
\hline$N_{6}^{\mathfrak{g}_{2}^{-\frac{2}{9}}}, N_{7}^{\mathfrak{g}_{2}^{-\frac{2}{9}}}, N_{9}^{\mathfrak{g}_{2}^{0}}, N_{11}^{\mathfrak{g}_{2}^{0}}$ & Always \\
$N_{1}^{\mathfrak{g}_{3}}(a), N_{2}^{\mathfrak{g}_{3}}(a), N_{5}^{\mathfrak{g}_{3}}$ & \\
\hline$N_{8}^{\mathfrak{g}_{2}^{0}}(a)$ & {$[a=-1]$ or $[a=-2]$} \\
\hline$N_{10}^{\mathfrak{g}_{2}^{0}}(a)$ & {$[a=-1]$} \\
\hline$N_{1}^{\mathfrak{g}_{4}}(a), N_{1}^{\mathfrak{g}_{5}}(a)$ & {$[a=0]$} \\
\hline
\end{tabular}

Table 5: Examples of infinitely balanced Lie algebras.

\begin{tabular}{l|l}
\hline The Lie algebra & Conditions on the Hermitian metric \\
\hline$N_{2}^{\mathfrak{g}_{3}}(a)$ & {$\left[g_{2,3}=0, g_{2,2}=g_{3,3}, 0<g_{3,3}, a<-\frac{g_{1,2}\left(g_{1,2}-g_{3,3}\right)}{g_{3,3}{ }^{2}}, g_{1,1}=-\frac{a g_{2,2} g_{3,3}-g_{1,2} g_{3,3}-g_{1,3}{ }^{2}}{g_{3,3}}\right]$} \\
\hline$N_{5}^{\mathfrak{g}_{3}}$ & Always \\
\hline$N_{1}^{\mathfrak{g}_{4}}(a), N_{1}^{\mathfrak{g}_{5}}(a)$ & {$\left[a=0, g_{1,2}=0, g_{1,3}=0,0<g_{1,1}, 0<g_{3,3}, \frac{g_{2,3}{ }^{2}}{g_{3,3}}<g_{2,2}\right]$} \\
\hline
\end{tabular}


Table 6: Examples of balanced non Kähler Lie algebras.

\begin{tabular}{|c|c|}
\hline The Lie algebra & Conditions on the Hermitian metric \\
\hline$N_{2}^{\mathfrak{g}_{2}^{\alpha}}(a)$ & $\begin{array}{l}{\left[a=-1, \alpha=0, g_{12}=\frac{g_{1,3} g_{2,3}}{g_{3,3}}, 0<g_{3,3}, \frac{g_{1,3}{ }^{2}}{g_{3,3}}<g_{1,1}, \frac{g_{2,3}{ }^{2}}{g_{3,3}}<g_{2,2}\right]} \\
{\left[a=-1, g_{1,2}=g_{1,3}=0,\left(g_{2,2}, g_{2,3}\right) \neq\left(-\frac{g_{3,3}}{\alpha}, \frac{g_{2,2}}{2}\right), 0<g_{1,1}, 0<g_{3,3}, \frac{g_{2,3}{ }^{2}}{g_{3,3}}<g_{2,2}\right],}\end{array}$ \\
\hline$N_{5}^{\mathfrak{g}_{2}^{-\frac{2}{9}}}(a)$ & $\begin{array}{l}{\left[a=-\frac{1}{3}, g_{1,1}=2 g_{1,3}, g_{1,2}=2 g_{1,3}, g_{2,3}=3 g_{1,3}, g_{3,3}=g_{1,3}, 0<g_{1,3}, 10 g_{1,3}<g_{2,2}\right]} \\
{\left[a=-\frac{1}{3}, g_{1,1}=2 g_{1,3}, g_{1,2}=3 g_{1,3}, g_{2,3}=3 g_{1,3}, g_{3,3}=g_{1,3}, 0<g_{1,3}, 9 g_{1,3}<g_{2,2}\right]}\end{array}$ \\
\hline$N_{8}^{\mathfrak{g}_{2}^{0}}(a)$ & {$\left[a=-2, g_{1,2}=0, g_{1,3}=0, g_{2,3} \neq g_{3,3}, 0<g_{1,1}, 0<g_{3,3}, \frac{g_{2,3}{ }^{2}}{g_{3,3}}<g_{2,2}\right]$} \\
\hline$N_{10}^{\mathfrak{g}_{2}^{0}}(a)$ & {$\left[a=-1, g_{1,2}=g_{1,3}, g_{2,3}=g_{3,3}, 0<g_{3,3}, g_{3,3}<g_{2,2}, \frac{g_{1,3}{ }^{2}}{g_{3,3}}<g_{1,1}\right]$} \\
\hline$N_{11}^{\mathfrak{g}_{2}^{0}}$ & {$\left[g_{1,2}=\frac{g_{1,3} g_{2,3}}{g_{3,3}}, g_{2,2}=\frac{g_{1,1} g_{2,3} g_{3,3}-g_{1,1} g_{3,3}{ }^{2}-g_{1,3}{ }^{2} g_{2,3}+g_{1,3}{ }^{2} g_{3,3}+g_{2,3}{ }^{2} g_{3,3}}{g_{3,3}{ }^{2}}, 0<g_{3,3}, g_{3,3}<g_{2,3}, \frac{g_{1,3}{ }^{2}}{g_{3,3}}<g_{1,1}\right.$} \\
\hline$N_{2}^{\mathfrak{g}_{3}}(a)$ & {$\left[g_{1,3}=g_{2,3}=0, g_{1,1}=-\frac{a g_{2,2} g_{3,3}-g_{1,2} g_{3,3}}{g_{3,3}}, a \neq 1,0<g_{2,2}, 0<g_{3,3}, \frac{a^{2} g_{2,2}{ }^{2}-2 a g_{1,2} g_{2,2}+g_{1,2}{ }^{2}}{g_{2,2}}<g_{1,1}\right]$} \\
\hline$N_{5}^{\mathfrak{g}_{3}}$ & Always \\
\hline$N_{1}^{\mathfrak{g}_{4}}(a), N_{1}^{\mathfrak{g}_{5}}(a)$ & {$\left[a=0, g_{1,2}=0, g_{1,3}=0,0<g_{1,1}, 0<g_{3,3}, \frac{g_{2,3}{ }^{2}}{g_{3,3}}<g_{2,2}\right]$} \\
\hline
\end{tabular}

Table 7: Examples of pluriclosed non Kähler Lie algebras.

\begin{tabular}{|c|c|}
\hline The Lie algebra & Conditions on the Hermitian metric \\
\hline$N_{1}^{\mathfrak{g}_{1}}(a)$ & {$\left[a=-2,\left(g_{1,2}, g_{1,3}\right) \neq(0,0)\right]$ or $[a=-1]$. } \\
\hline$N_{2}^{\mathfrak{g}_{1}}$ & Always \\
\hline$N_{2}^{\mathfrak{g}_{2}^{\alpha}}(a)$ & $\begin{array}{l}{\left[a=-\frac{2}{3}, \alpha=-\frac{2}{9}, g_{1,3}=0, g_{3,3}=-\frac{2 g_{2,2}}{9}+g_{2,3}, 0<g_{2,3}, g_{2,2}<3 g_{2,3},\right.} \\
\left.\frac{g_{1,2}{ }^{2}\left(2 g_{2,2}-9 g_{2,3}\right)}{2 g_{2,2^{2}}-9 g_{2,2} g_{2,3}+9 g_{2,3}{ }^{2}}<g_{1,1}, \frac{3}{2} g_{2,3}<g_{2,2}\right] \\
{\left[a \in\left\{-\frac{1}{2},-1\right\}, g_{1,3}=0, g_{2,3}=\frac{1}{2} g_{2,2}, g_{3,3}=-\alpha g_{2,2}, 0<g_{2,2}, \alpha<-\frac{1}{4} \frac{g_{2,2} g_{1,1}}{g_{2,2} g_{1,1}-g_{1,2}}, \frac{g_{1,2}}{g_{2,2}}<g_{1,1}\right]}\end{array}$ \\
\hline$N_{2}^{\mathfrak{g}_{2}^{a^{2}+a}}(a)$ & {$\left[a=-\frac{2}{3}, g_{3,3}=-\frac{2}{9} g_{2,2}+g_{2,3}, g_{1,2}=0,0<g_{2,3}, g_{2,2}<3 g_{2,3}, \frac{3}{2} g_{2,3}<g_{2,2},-\frac{9 g_{1,3}{ }^{2} g_{2,2}}{2 g_{2,2}-9 g_{2,2} g_{2,3}+9 g_{2,3}{ }^{2}}<g_{1,1}\right.$} \\
\hline$N_{6}^{\mathfrak{g}_{2}^{-\frac{2}{9}}}$ & {$\left[g_{2,2}=\frac{9}{2}\left(g_{2,3}-g_{3,3}\right), g_{1,2}=0,0<g_{2,3}, g_{2,2}<3 g_{2,3}, \frac{3}{2} g_{2,3}<g_{2,2},-\frac{9 g_{1,3}{ }^{2} g_{2,2}}{2 g_{2,2^{2}-9} g_{2,2} g_{2,3}+9 g_{2,3}{ }^{2}}<g_{1,1}\right]$} \\
\hline$N_{8}^{\mathfrak{g}_{2}^{0}}(a), N_{9}^{\mathfrak{g}_{2}^{0}}$ & {$\left[a \in\{-1,-2\}, g_{1,3}=0, g_{2,3}=g_{3,3}, 0<g_{1,2}, 0<g_{3,3}, g_{1,2}<g_{3,3}, g_{3,3}<g_{2,2}, \frac{g_{1,2}{ }^{2}}{g_{2,2}-g_{3,3}}<g_{1,1}\right]$} \\
\hline$N_{10}^{\mathfrak{g}_{2}^{0}}(a)$ & $\begin{array}{l}{\left[a=-2, g_{1,2}=g_{1,3}+2 g_{2,2}-2 g_{2,3}, g_{3,3}=g_{2,3}, 0<g_{3,3}, g_{3,3}<g_{2,2}, \frac{g_{1,3}{ }^{2}+4 g_{2,2} g_{3,3}-4 g_{3,3}{ }^{2}}{g_{3,3}}<g_{1,1}\right]} \\
{\left[a=-1, g_{1,2}=g_{1,3}+g_{2,2}-g_{2,3}, g_{3,3}=g_{2,3}, 0<g_{3,3}, g_{3,3}<g_{2,2}, \frac{g_{1,3}{ }^{2}+g_{2,2} g_{3,3}-g_{3,3}{ }^{2}}{g_{3,3}}<g_{1,1}\right]}\end{array}$ \\
\hline$N_{11}^{\mathfrak{g}_{2}^{0}}$ & {$\left[g_{1,2}=g_{1,3}+g_{2,2}-g_{2,3}, g_{3,3}=g_{2,3}, 0<g_{3,3}, g_{3,3}<g_{2,2}, \frac{g_{1,3}{ }^{2}+g_{2,2} g_{3,3}-g_{3,3}{ }^{2}}{g_{3,3}}<g_{1,1}\right]$} \\
\hline$N_{2}^{\mathfrak{g}_{3}}(a)$ & {$[a=1]$} \\
\hline$N_{1}^{\mathfrak{g}_{5}}(a)$ & {$\left[a=0, g_{2,2}=g_{3,3}, g_{2,3}=0,0<g_{2,2}, \frac{g_{1,2}{ }^{2}+g_{1,3}{ }^{2}}{g_{2,2}}<g_{1,1}\right]$} \\
\hline
\end{tabular}


Table 8: Examples of Calabi-Yau with torsion Lie algebras which are not infinitely balanced

\begin{tabular}{|c|c|}
\hline The Lie algebra & Conditions on the Hermitian metric \\
\hline$N_{1}^{\mathfrak{g}_{1}}(a)$ & {$\left[a=2, g_{1,2}=0, g_{1,3}=0,0<g_{1,1}, 0<g_{3,3}, \frac{g_{2,3}^{2}}{g_{3,3}}<g_{2,2}\right]$} \\
\hline$N_{2}^{\mathfrak{g}_{2}^{\alpha}}(a)$ & $\begin{array}{l}{\left[a=1, \alpha=2, g_{1,2}=-\frac{2 g_{2,2} g_{1,3}}{g_{3,3}}, g_{2,3}=0,0<g_{2,2}, 0<g_{3,3}, \frac{\left(4 g_{2,2}+g_{3,3}\right) g_{1,3}{ }^{2}}{g_{3,3^{2}}}<g_{1,1}\right]} \\
{\left[a=1, g_{1,2}=0, g_{1,3}=0,0<g_{1,1}, 0<g_{3,3}, \frac{g_{2,3}}{g_{3,3}}<g_{2,2}\right]} \\
{\left[a=1, \alpha=2, g_{1,2}=0, g_{2,3}=2 g_{2,2}, 0<g_{2,2}, g_{1,3}<-\frac{5}{2} g_{2,2}, 4 g_{2,2}<g_{3,3},-\frac{g_{1,3}^{2}}{-g_{3,3}+4 g_{2,2}}<g_{1,1}\right]} \\
{\left[a=1, \alpha=2, g_{1,3}=-g_{1,2}, g_{2,2}=-\frac{1}{2}\left(g_{2,3}-g_{3,3}\right), 0<g_{3,3}, g_{2,3}<\frac{1}{2} g_{3,3},-g_{3,3}<g_{2,3},-\frac{3 g_{1,2}^{2}}{2 g_{2,3}-g_{3,3}}<g_{1,1}\right.}\end{array}$ \\
\hline$N_{5}^{\mathfrak{g}_{2}^{-\frac{2}{9}}}(a)$ & 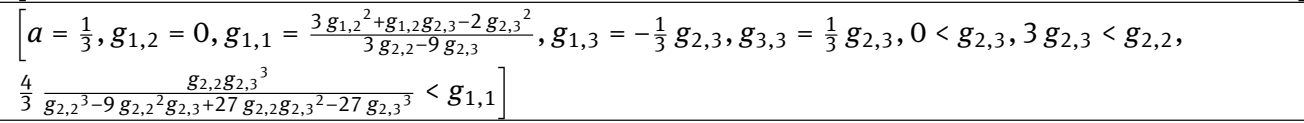 \\
\hline$N_{8}^{\mathfrak{g}_{2}^{0}}(a)$ & {$\left[a=1, g_{1,2}=0, g_{1,3}=0,0<g_{3,3}, \frac{g_{2,3}{ }^{2}}{g_{3,3}}<g_{2,2}\right]$} \\
\hline$N_{10}^{\mathfrak{g}_{2}^{0}}(a)$ & $\begin{array}{l}{\left[a=1, g_{1,1}=-g_{1,2}, g_{1,3}=0, g_{2,3}=g_{3,3}, 0<g_{2,3}, g_{1,2}<0,-g_{1,2}+g_{2,3}<g_{2,2}\right]} \\
{\left[a=1, g_{1,1}=-\frac{g_{1,2} g_{3,3}-g_{1,3}{ }^{2}-g_{1,3} g_{2,3}}{g_{3,3}}, g_{2,2}=\frac{g_{1,2} g_{2,3}-g_{1,2} g_{3,3}+g_{1,3} g_{2,3}}{g_{1,3}}, 0<g_{3,3}, g_{1,2}<\frac{g_{1,3} g_{2,3}}{g_{3,3}},\right.} \\
\left.g_{1,3}<0, g_{3,3}<-2 g_{1,3}-\sqrt{2} \sqrt{g_{1,3}{ }^{2}}, g_{3,3}-g_{1,3}<g_{2,3}\right]\end{array}$ \\
\hline
\end{tabular}

Acknowledgment: I would like to thank the referee for having read the paper carefully and giving us substantial remarks and suggestions which will improve the paper drastically. I would also like to thank Oumaima Tibssirte for having read and made many corrections to the first version of the paper.

Conflict of interest: Author state no conflict of interest.

\section{References}

[1] Alexandrov B., Ivanov S., Vanishing theorems on Hermitian manifolds, Differ. Geom. Appl. 14(3). 251-265 (2001).

[2] Alekseevskii D. and Kimel'fel'd B., Structure of homogeneous Riemann spaces with zero Ricci curvature Functional Anal Appl. 9 (1975), 97-102.

[3] Angella D., Otal A., Ugarte L., Villacampa, R., On Gauduchon connections with Kähler-like curvature, Commun. Anal. Geom (2018) (to appear).

[4] Arroyo R.M., Lafuente R., The long-time behavior of the homogeneous pluriclosed flow, Proc. Lond. Math. Soc. 3(119), 266-289 (2019).

[5] A. BesSE, Einstein manifolds, Springer-Verlag, Berlin-Hiedelberg-New York (1987).

[6] Bismut J.-M., A local index theorem for non-Kähler manifolds, Math. Ann. 284(4),681-699 (1989).

[7] Burde D., de Graaf W., Classification of Novikov algebras, Applicable Algebra in Engineering, Communication and Computing volume 24, 1 ?15 (2013).

[8] S. Cohn-Vossen, Totalkrummung und geodatische Linien auf einfach zusammenhangenden offenen vollstandigen Flachenstucken, Recueil Math. de Moscou 43 (1936), 139-163.

[9] A. Cosgaya and S. Reggiani, Isometry groups of three-dimensional Lie goups, arXiv:2105.08924 [math.DG].

[10] Fino A., Parton M., Salamon S., Families of strong KT structures in six dimensions, Comment. Math. Helv. 79(2), 317-340 (2004).

[11] Gauduchon P., La 1-forme de torsion d'une variété hermitienne compacte, Math. Ann. 267(4), 495-518 (1984).

[12] Gauduchon P., Hermitian connections and Dirac operators, Boll. Un. Mat. Ital. B (7) 11(2), 257-288 (1997).

[13] E. Ghys, Classification des feuilletages totalement géodésiques de codimension un, Comment. Math. Helvetici 58 (1983) $543-$ 572.

[14] K. Y. Ha and J. B. Lee, The isometry groups of simply connected 3-dimensional unimodular Lie groups, J. Geom. Phys. 62 (2012), 189-203.

[15] K. Y. Ha and J. B. Lee, Left invariant metrics and curvatures on simply connected three-dimensional Lie groups, Math. Nachr. 282 (2009), no. 1, 868-898.

[16] Helmstetter, J., Radical d'une algèbre symétrique à gauche, Annales de l'Institut Fourier 29 (1979) 17-35. 
[17] C. Hull, Superstring compactifications with torsion and space-time supersymmetry, In Turin 1985 Proceedings "Superunification and Extra Dimension" (1986), 347-375.

[18] S. Kobayashi and K. Nomizu, Foundations of Differential Geometry, Volume II, A Wiley Interscience Publication (1996).

[19] B. Kostant, A characterization of invariant affine connections, Nagoya Math. J. 16: 35-50 (1960).

[20] J. Li, S.-T. Yau, The existence of supersymmetric string theory with torsion, J. Diff. Geom. 70 (2005), no. 1, 143-181.

[21] Milnor, J., Curvatures of left invariant metrics on Lie Groups, Advances in Mathematics 21 (1976), 293-329.

[22] Moroianu A., Moroianu S., Ornea, L., Locally conformally Kähler manifolds with holomorphic Lee field, Differ. Geom. Appl. 60, 33-38 (2018).

[23] H. Shima. The geometry of Hessian structures. World Scientific Publishing (2007).

[24] A. Strominger, Superstrings with torsion, Nucl. Phys. B 274 (1986), no. 2, 253-284.

[25] Ugarte L., Hermitian structures on six-dimensional nilmanifolds, Transform. Groups 12(1), 175-202 (2007).

[26] Wang Q., Yang B., Zheng F., On Bismut flat manifolds, Trans. Amer. Math. Soc. 373 (2020), 5747 ?5772.

[27] Yang, B., Zheng, F., On curvature tensors of Hermitian manifolds, Comm. Anal. Geom. 26 (2018), 1193- 1220.

[28] Zhao Q., Zheng F., Strominger connection and pluriclosed metrics, (2019). arXiv:1904.06604[math.DG]. 